\title{
Secondary structural entropy in RNA switch (Riboswitch) identification
}

\author{
Amirhossein Manzourolajdad ${ }^{1,2^{*}}$ and Jonathan Arnold ${ }^{1,3}$
}

\author{
${ }^{*}$ Correspondence: \\ amanzour@uga.edu \\ ${ }^{1}$ Institute of Bioinformatics, \\ University of Georgia, Davison Life \\ Sciences Bldg, Room B118B, 120 \\ Green St, 30602 Athens, USA \\ ${ }^{2}$ National Center for Biotechnology \\ Information (NCBI), NIH, Building \\ 38A, RM 6S614K, 8600 Rockville \\ Pike, 20894 Bethesda, USA \\ Full list of author information is \\ available at the end of the article
}

\begin{abstract}
Background: RNA regulatory elements play a significant role in gene regulation. Riboswitches, a widespread group of regulatory RNAs, are vital components of many bacterial genomes. These regulatory elements generally function by forming a ligand-induced alternative fold that controls access to ribosome binding sites or other regulatory sites in RNA. Riboswitch-mediated mechanisms are ubiquitous across bacterial genomes. A typical class of riboswitch has its own unique structural and biological complexity, making de novo riboswitch identification a formidable task. Traditionally, riboswitches have been identified through comparative genomics based on sequence and structural homology. The limitations of structural-homology-based approaches, coupled with the assumption that there is a great diversity of undiscovered riboswitches, suggests the need for alternative methods for riboswitch identification, possibly based on features intrinsic to their structure. As of yet, no such reliable method has been proposed.
\end{abstract}

Results: We used structural entropy of riboswitch sequences as a measure of their secondary structural dynamics. Entropy values of a diverse set of riboswitches were compared to that of their mutants, their dinucleotide shuffles, and their reverse complement sequences under different stochastic context-free grammar folding models. Significance of our results was evaluated by comparison to other approaches, such as the base-pairing entropy and energy landscapes dynamics. Classifiers based on structural entropy optimized via sequence and structural features were devised as riboswitch identifiers and tested on Bacillus subtilis, Escherichia coli, and Synechococcus elongatus as an exploration of structural entropy based approaches. The unusually long untranslated region of the $\mathrm{cotH}$ in Bacillus subtilis, as well as upstream regions of certain genes, such as the sucC genes were associated with significant structural entropy values in genome-wide examinations.

Conclusions: Various tests show that there is in fact a relationship between higher structural entropy and the potential for the RNA sequence to have alternative structures, within the limitations of our methodology. This relationship, though modest, is consistent across various tests. Understanding the behavior of structural entropy as a fairly new feature for RNA conformational dynamics, however, may require extensive exploratory investigation both across RNA sequences and folding models.

Keywords: Riboswitch, Entropy, RNA secondary structure, cotH, sucC 


\section{Background}

Non-protein-coding RNA (ncRNA) elements play an important role in biological pathways, such as gene regulation [1-4]. It has been shown that conformational features of many such RNA elements play a major part in their biological function [5,6]. In bacteria, RNA structural rearrangements can have a major effect on the expression of their downstream coding sequences (reviewed by [7]), a process known as cis-regulation. A classic example, and one of the earliest such elements discovered, is the complex regulatory mechanism that takes place upstream of the tryptophan operon in Escherichia coli during its expression [8]. Regulation of the tryptophan biosynthetic operon, however, is achieved through very different mechanisms in other organisms, such as B. subtilis and Lactobacillus lactis (reviewed by [9]). With much attention given to protein-coding genes in the past, the introduction of ncRNAs gene finders have become a relatively new area of genomic research [10]. Currently, many general-purpose [11-13] as well as ncRNA-specific gene finders, such as [14-16] are available.

\section{Riboswitches}

An interesting group of RNA regulatory elements are riboswitches. Riboswitches are defined as regulatory elements that take part in biological pathways by selectively binding to a specific ligand or metabolite, or uncharged tRNAs, without the need for protein factors. Environmental factors such as $\mathrm{pH}$ [17], ion concentration [18-20], and temperature $[21,22]$ can also trigger RNA conformational changes affecting gene regulation. Nearly all riboswitches are located in the non-coding regions of messenger RNAs [23] and are capable of regulating genes through both activation and attenuation of either transcription or translation (reviewed by [24]). Finally, other factors such as the transcription speed of RNA polymerase, the folding and unfolding rates of the aptamer of the riboswitch, and the binding rates of the metabolites add other dimensions to categorizing riboswitches. These and other factors influence the RNA switching mechanism to be kinetically or thermodynamically driven. In addition to thermodynamics-based approaches, RNA-kinetics have been gaining momentum in riboswitch-mediated regulation studies at the system level. Lin and Thirumalai [25] introduces a kinetic feedback-loop network model that describes the functions of riboswitches using experimental data from flavin mononucleotide (FMN) riboswitch.

Originally found through sequence homology upstream of bacterial coding regions [26-28], riboswitches have been shown to be more abundant than previously expected. They have also been found in cooperative or tandem arrangements [23]. It is speculated that there are at least 100 more undiscovered riboswitches in already sequenced bacterial genomes [23]. Conformational factors are essential to ligand-binding specificity of riboswitches. Many riboswitches can discriminate between similar small molecules with the aid of their structural geometry. For instance, the thiamine pyrophosphate (TPP) and $\mathrm{S}$-adenosylmethionine (SAM) riboswitches measure the length of the ligand that binds to them [29-31].

\section{RNA secondary structure}

The secondary structural topology of the RNA is very effective in scaffolding the tertiary conformation. Secondary structure mainly consists of a two-dimensional schema that depicts the base-pairing interactions within the RNA structure and is dominated by 
Watson-Crick base-pairing. One major computational method to predict RNA secondary structure is minimization of its free energy (MFE) within a thermodynamic ensemble, such as the Boltzmann ensemble [32,33]. State-of-the-art thermodynamic models have proven to be effective in RNA secondary structural predictions in most cases. An example of where such predictions fail would be Hammerhead type I ribozyme where loop tertiary interactions have a dominating effect on the structural conformation [34]. Centroids of the Boltzmann ensemble are also used for RNA secondary structural predictions [35]. In many cases, such a prediction is more similar to the structure inferred from comparative sequence analysis than the MFE structure is [35]. In addition, Stochastic context-free grammars (SCFG) have shown to be effective in secondary structural prediction of various RNA regulatory elements. Nawrocki and Eddy, 2013 [13] have shown that more sophisticated grammars, designed to mirror the thermodynamic models can improve the prediction accuracy of structures, once trained on known RNA structures based on maximum-likelihood criteria ${ }^{\mathrm{a}}$.

Most of the discovered prokaryotic RNA regulatory elements (including riboswitches) are located upstream of the genes they regulate. They act as cis-regulatory elements and exhibit strong secondary structural conservation. Some exceptions to cis-regulation are two trans-acting SAM riboswitches [36] and an antisense regulation of a vitamin $B_{12}$ binding riboswitch [37] in Listeria monocytogenes. Insights into structural and functional complexity of riboswitches already discovered are offered in [38]. Purine riboswitches are good examples of secondary structural conservation. The add adenine riboswitch from $V$. vulnificus and the xpt guanine riboswitch from B. subtilis have very similar secondary and tertiary conformations, despite different crystal packing interactions, $\mathrm{pH}$, and $\mathrm{Mg}$ crystallization conditions [39]. In fact, investigation of secondary-structural homology upstream of genomic regions containing the same genes has led to the discovery of more cis-regulatory elements in bacteria [40,41], making them the major current approach for riboswitch identification.

The fact that riboswitch discovery is mainly based on homology makes it difficult to assess how much secondary structural conservation is expected to be prevalent in undiscovered riboswiches. Furthermore, structural homology is not always successful in finding riboswitches. Despite [42]'s rigorous sequence and structural homology searches based on the SAM-I riboswitch, the SAM-IV riboswitch could not be detected. The authors further hypothesized that the structural diversity of riboswitches could be far greater than what has been already observed. Serganov and Nudler, 2013 [38] suggest that there may not even be an interconnection between the structures of riboswitches and the nature of their cognate metabolites and consequently, the biochemical and structural information gathered so far may not be as useful in riboswitch validation as expected. The above limitations of homology-based riboswitch identification methods indicate the need for an alternative approach.

\section{Conformational dynamics}

While secondary-structure conformational features are very descriptive of many classes of riboswitches, their folding dynamics are also critical. A typical example is the TPP riboswitch which can fold into alternative structures depending on the presence of the TPP ligand. The tertiary structure stabilized in the presence of TPP is shown in Figure 1A [43]. Both the ligand-bound and the unbound secondary structures necessary for TPP 


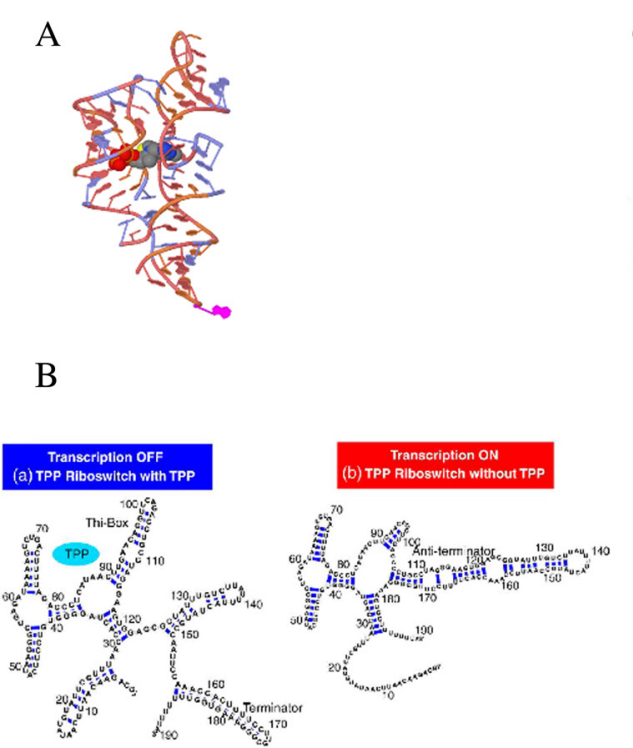

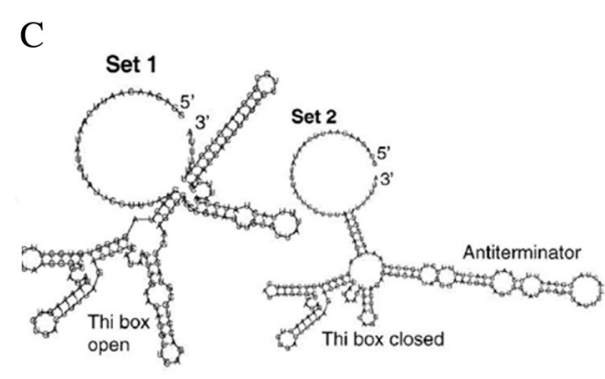

Energy landscape of 190 nucleotide TPP riboswitch

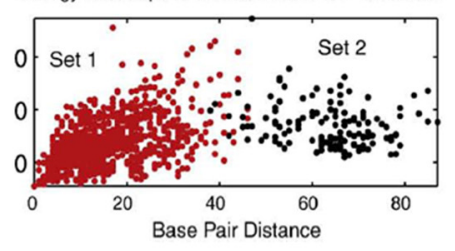

Figure 1 Energy Landscape of The TPP Riboswitch. A: Tertiary structure of an E. coli TPP (or thi-box) riboswitch bound to thiamine pyrophosphate [43]. The image was generated by the Jmol from the PDB:2hoj structure taken from the Rfam website [89]. B: Ligand-bound and unbound secondary structures of a TPP riboswitch in B. subtilis, taken from [45]. C: Energy landscape of the B. subtilis riboswitch taken from [45]. Set-1 and Set-2 clusters correspond to the two mutually exclusive secondary structures of the TPP riboswitch. Pairwise Base-pairing distance used as a measure of distance between two structures. Please refer to [45] for detailed information about the figure and clustering details.

riboswitch regulatory function are shown in Figure 1B. One of the major computational tools to explore possible folding trajectories is the free energy landscape. The free energy landscape was originally defined for protein folding [44]. In a typical RNA free energy landscape, possible conformations are shown with their corresponding free energy and pairwise distances from one another. In an effort to investigate the thermodynamic equilibrium of RNA folding, Quarta et al. [45] presented a case study of the energy landscape of the TPP riboswitch where the base-pairing distances between the structural possibilities form two major clusters. The clusters corresponded to native and ligand-bound structural conformations. After repeating this process for various choices of elongation of the TPP riboswitch, they showed that for certain ranges of length, each cluster corresponds to one of the two structures of the riboswitch (see Figure 1C).

In [46], the dynamics of energy landscapes across elongation of various riboswitches were investigated and it was shown that such landscapes have different clustering dynamics across kinetically and thermodynamically driven riboswitches. This work highlights the fact that even in a kinetically-driven regulation scenario, investigation of the dynamics of the thermodynamic equilibrium across the elongation can be informative. In a more recent work, energy landscape analyses led to strong evidence of evolutionary co-variation of base-pairs that favor a conserved alternative structure of the purine riboswitch [47]. In addition, prediction of structural switching in RNA has been addressed by $[48,49]$ using abstract shapes to represent different secondary structural conformations. Freyhult et al. 2007 [50,51] examined the lowest free energy structural conformations having a certain base-pairing distance to the actual structure of the RNA to explore the structural neighbors of an intermediate, biologically active structure. A 
more recent work [52] presents an ingenious and a significant decrease of computational consumption of estimating the likelihood of structural neighbors. However, to date there is no computational method that can identify the diverse and structurally complex riboswitches with high confidence.

Investigation into the folding dynamics of the nascent RNA based on free energy sampling and pair-wise distances can be computationally costly. Finding a sample size that sufficiently reflects the RNA folding space behavior can be difficult and prone to model parameter biases. Furthermore, even if optimized parameters and sufficient samples were available, it would still be difficult to make comparisons across RNA elements. The latter is mainly due to the fact that the characteristics of such folding distributions (here, free energy vs. structural distance within a given ensemble of secondary structures) are not well understood.

One statistic to evaluate the distribution characteristics of any probabilistic model is the Shannon entropy [53]. While the conformation with maximum-likelihood under a given SCFG is referred to as the optimum structure under that model, all of the other sub-optimal conformations can be associated with a probability. Hence, the Shannon entropy (expected log-likelihood) of such a probabilistic folding space is $H(S)=$ $-\sum_{s \in S} p(s) \log p(s)$, where $S$ is the folding space containing all possible secondary structures $s$ valid on the desired RNA sequence, each of which associated with the corresponding probabilities of occurrence $p(s)$. Here, the notion of probability can also be interpreted as the frequency of occurrence of a particular conformation for the RNA sequence. Alternative formulations and approximations of Shannon entropy exist in RNA secondary structure studies, such as [54]. Exact calculations of Shannon entropy under a given SCFG as a probabilistic secondary structural folding model, however, was done in [55] and shown to be computationally convenient achievable in polynomial time $O\left(n^{3}\right)$, where $n$ is the length of the RNA sequence. In an independent work, [56] also offered an algorithm to calculate the Shannon entropy of the stochastic context-free grammar BJK [57] with parameter sets derived from a given alignment. Other measures of structural diversity such as ensemble diversity computed by RNAfold -p in the Vienna RNA Software Package [58] also exist. In this work, structural diversity is measured by the exact RNA secondary structural information theoretic-uncertainty (or here, Shannon entropy) of the complete SCFG-modeled folding space of the RNA, as computed by [55]. From hereon, we refer to this measure as structural entropy. We investigated the significance of structural entropy of RNAs with more than one biologically functional secondary structural conformation. A diverse set of prokaryotic RNA elements, validated to have such potential were used for this purpose. The performance of structural entropy to distinguish riboswitches was compared to other similar features under different negative-control sets. We then made an attempt to develop a computational method for riboswitch identification via structural entropy on a genome-wide level. The goal of the presented results of the genome-wide tests, however, is mainly exploratory and aim to investigate the genomic regions or elements that the developed method is highly sensitive to.

It has been previously shown that both high and low structural entropy values of certain classes of ncRNAs can be potentially significant. For instance, for certain riboswitches, GC-composition was co-associated with significantly high structural entropy, regardless of model accuracy to RNA secondary structure [55]. This 
observation raised the possibility that RNAs under selective pressure to have alternative folds, may have higher (not lower) structural entropy than expected. As discussed previously in [55], this seemingly nonintuitive observation is not theoretically impossible. The above intuition lies at the center of the proposed methodology, as will be shown.

\section{Our approach}

\section{Folding models}

The folding model for which the structural entropy of the RNA is computed is very critical. SCFG folding models can be very lightweight and consist of only few grammar rules and parameters, or they can be very sophisticated consisting of thousand parameters $[13,59]$. In [55], it was shown that the structural entropy value is very model sensitive. On the other hand, parameters of SCFG models are usually set by maximizing their prediction accuracy using maximum-likelihood approaches. There is no guarantee, however, that folding models optimized for such criteria also preserve information about folding dynamics of such RNAs. Increasing the accuracy of folding models under current approaches may be done at the expense of altering the folding space of possible structures under that model, thus losing the information about folding dynamics of the RNA. In order to avoid potential biases in our preliminary examination, it was essential that we include models not trained to best predict secondary structure in addition to models that do. Two different SCFG models were chosen for this study, one being a structurally unambiguous SCFG model with parameters trained to best predict RNA secondary structure, and one being a structurally ambiguous model with symmetric rules and probabilities. The theoretical implications of structural ambiguity may fall outside the scope of this work and the interested reader can refer to [55]. Here, we merely treat them as two different folding models.

\section{Gathering data}

There is a significant amount of sequence and/or structural similarity within each class of riboswitch. This is due to the fact that these riboswitches have been discovered using sequence and/or structural homology. Here, however, we are interested in capturing the universal characteristics of RNAs with alternative fold(s), mainly riboswitches, as a basis for an identification method for conformational switches. In order for our method to be less biased towards a specific structural conformation, we avoided using homologous RNA sequences or sequences that belong to closely related organisms, where possible. We also resorted to only evaluating riboswitches that have been experimentally validated to be functional rather than computationally discovered ones. The data set gathered in this work is a compromise between the above considerations and the need to include a diverse set of riboswitches in our data set. Although the attempt to computationally extract a universal feature from the diversity of prokaryotic riboswitches each having unique structural and biological characteristics is a great oversimplification, it serves as a common ground for comparing various features that aim to capture the RNA conformational dynamics as a whole.

\section{Negative controls}

One of the main challenges of our test, was the preparation of a reliable negative control. Folding models deployed here are very lightweight and simplistic, giving rise to potential 
unrelated dependencies to the factors such as genomic composition of RNA sequence. Therefore, gathering real biological sequences that are as similar to RNA sequences as possible while not having potential for alternative fold(s) is very critical to the significance of our test. Here, we relied on the following sets of negative controls: 1. dinucleotide shuffles of riboswitches (generated using [60]), 2. Mutagenesis; Structural mutants of the gathered sequences experimentally tested for not being functional, 3 . The reverse complements (or antisense sequences) of gathered riboswitches, and 4. Sequences of the non-coding regions that are likely to be riboswitches. The choice of antisense as a negative control is explained in the Methods section.

\section{Comparison to other methods}

Two additional measures of structural diversity were used to assess the significance of structural entropy values in collected data. The first measure was the base-pairing entropy [54] of the BJK model BJKbp as defined in ([61] Eq. 3). For more information see the Methods section. The second measure, denoted as Sil, was obtained from clustering the RNA energy landscape. The Sil value reflects how well the energy landscape clusters into two. Calculations for Sil were according to [46]. We then compared the performance of classifiers designed to distinguish riboswitches from various negative controls. In order to evaluate the performance of structural entropy to detect alternative fold, we compared it to measures from RNAShapes [49] and FFTbor [52] predictions. These measures corresponded to energy disequilibrium of alternative folds: $p 1 / p 2$, where $p 1$ was the highest value in the predictions of the corresponding software and $p 2$ was the second highest value. For RNAShapes, $p 1$ is the probability of the most likely abstract shape of structure, whereas $p 2$ is the second most. For FFTbor, $p 1$ is the probability of the MFE structure and $p 2$ is the probability of an alternative folding scenario where the structure has a particular base-pair distance with the MFE structure. Features used in this work are shown in Table 1. Please see the Methods section for further details.

\section{Results and discussion}

The two lightweight SCFG folding models used to calculate structural entropy are denoted here as BJK and RND models, which are taken from the literature (Please see the Methods section). RNA encoded sequence from Bacteria validated to have potential for two alternative folds were gathered from the literature (see Table 2) as representatives of RNAs having potential for alterative folding. This generally consisted of riboswitches and

Table 1 List of various sequence and structural features used throughout the work

\begin{tabular}{ll}
\hline Feature & Cardinality \\
\hline RND & Real positive \\
BJK & Real positive \\
BJKbp & Real positive \\
Sil & Real positive \\
FFTbor & Real positive \\
RNAShapes & Real positive \\
GC & Real positive less than one \\
MFE & Real negative \\
CFE & Real negative \\
L & Real positive \\
\hline
\end{tabular}


Table 2 Data collection

\begin{tabular}{|c|c|c|c|c|c|}
\hline ID & Riboswitch & Organism (P/N) & Alteration & Grouping & References \\
\hline ID01 & Alpha Operon & Escherichia coli (N) & Slow-fast & Train & {$[90,91]$} \\
\hline \multirow[t]{2}{*}{ ID02 } & ATP & Bacillus subtilis (P) & Enzyme & Test & [92] \\
\hline & $\operatorname{ATP}^{[1]}$ & Salmonella (N) & Enzyme & None & [73] \\
\hline ID03 & c-di-GMP & Geobacter sulfurreducens (N) & Ligand & Train & [40] \\
\hline ID04 & c-di-GMP & Candidatus Desulforudis (P) & Ligand & Test & {$[93]$} \\
\hline ID05 & Cobalamin & Escherichia coli (N) & Ligand & Train & [27] \\
\hline ID06 & Cobalamin & Bradyrhizobium japonicum (N) & Ligand & Train & [94] \\
\hline \multirow[t]{2}{*}{ ID07 } & Cobalamin & Salmonella $(\mathrm{N})$ & Ligand & Test & [95] \\
\hline & D. peptide ${ }^{[2]}$ & Synechococcus sp. CC9902 (N) & Motif & None & {$[96]$} \\
\hline ID08 & Fluoride & Pseudomonas syringae $(\mathrm{N})$ & Ligand & Train & [97] \\
\hline ID09 & Fluoride & Thermotoga petrophila $(\mathrm{N})$ & Ligand & Train & [98] \\
\hline ID10 & Fluoride & Bacillus cereus $(P)$ & Ligand & Test & {$[97]$} \\
\hline ID11 & FMN & Fusobacterium nucleatum (N) & Ligand & Train & {$[99,100]$} \\
\hline ID12 & FMN & Escherichia coli (N) & Ligand & Train & {$[20,101]$} \\
\hline \multirow[t]{3}{*}{ ID13 } & FMN & Bacillus subtilis (P) & Ligand & Test & [99-101] \\
\hline & glmS & T. tengcongensis $(\mathrm{N})$ & None & None & {$[75,76,102,103]$} \\
\hline & $g \ln A$ & Synechococcus elongatus (N) & Motif & None & [96] \\
\hline ID14 & Glycine & Fusobacterium nucleatum (N) & Ligand & Train & [104-106] \\
\hline \multirow[t]{3}{*}{ ID15 } & Glycine & Bacillus subtilis (P) & Ligand & Test & [104] \\
\hline & Hammerhead I & Schistosoma Mansoni (-) & None & None & {$[34,107]$} \\
\hline & Hammerhead II & Marine metagenome (-) & None & None & [108] \\
\hline ID16 & Lysine & Thermotoga maritima $(\mathrm{N})$ & Ligand & Train & {$[109,110]$} \\
\hline ID17 & Lysine & Bacillus subtilis $(\mathrm{P})$ & Ligand & Test & [110] \\
\hline ID18 & Magnesium & Salmonella enterica $(\mathrm{N})$ & $\mathrm{Mg}^{+}$ & Train & {$[18,20]$} \\
\hline ID19 & Magnesium & Escherichia coli (N) & $\mathrm{Mg} 2^{+}$ & Train & [18] \\
\hline ID20 & Magnesium & Bacillus subtilis (P) & $\mathrm{Mg} 2^{+}$ & Test & [19] \\
\hline ID21 & Moco & Escherichia coli (N) & Ligand & Train & [111] \\
\hline ID22 & pH-responsive & Escherichia coli (N) & $\mathrm{pH}$ & Train & [17] \\
\hline ID23 & pH-responsive & Serratia marcescens (N) & $\mathrm{pH}$ & Test & [17] \\
\hline ID24 & preQ1 ॥ & Streptococcus pneumoniae (P) & Ligand & Train & {$[40,112]$} \\
\hline ID25 & preQ1 I & Bacillus subtilis $(P)$ & Ligand & Test & [113] \\
\hline ID26 & Purine (Adenine) & Vibrio vulnificus (N) & Ligand & Train & [39] \\
\hline ID27 & Purine (Adenine) & Bacillus subtilis (P) & Ligand & Test & [39] \\
\hline ID28 & Purine (Guanine) & Bacillus subtilis (P) & Ligand & Test & {$[39,114]$} \\
\hline ID29 & ROSE-1 & Bradyrhizobium japonicum (N) & Heat & Train & {$[21,22]$} \\
\hline ID30 & ROSE-2 & Escherichia coli $(\mathrm{N})$ & Heat & Train & {$[21]$} \\
\hline ID31 & ROSE-2387 & Mesorhizobium loti (N) & Heat & Test & [21] \\
\hline ID32 & ROSE-N1 & Rhizobium (N) & Heat & Test & {$[21]$} \\
\hline ID33 & ROSE-P2 & Bradyrhizobium (N) & Heat & Train & {$[21]$} \\
\hline ID34 & $\mathrm{SAH}$ & Ralstonia solanacearum (N) & Ligand & Train & {$[40,115]$} \\
\hline ID35 & SAM-I & T. tengcongensis $(\mathrm{N})$ & Ligand & Train & [31] \\
\hline ID36 & SAM-I & Bacillus subtilis (P) & Ligand & Test & [116-119] \\
\hline ID37 & SAM-II & Agrobacterium tumefaciens (N) & Ligand & Train & [120] \\
\hline ID38 & SAM-III (SMK) & Streptococcus gordonii (P) & Ligand & Train & [121] \\
\hline ID39 & SAM-III (SMK) & Enterococcus faecalis (P) & Ligand & Test & [121-123] \\
\hline ID40 & SAM-IV & Streptomyces coelicolor (P) & Ligand & Train & {$[42]$} \\
\hline ID41 & SAM-IV & Mycobacterium tuberculosis (P) & Ligand & Test & [42] \\
\hline ID42 & SAM-SAH & Roseobacter $(\mathrm{N})$ & Ligand & Train & [41] \\
\hline ID43 & SAM-SAH & Oceanibulbus indolifex (N) & Ligand & Test & [41] \\
\hline ID44 & SAM-V & Cand. P. ubique $(\mathrm{N})$ & Ligand & Train & [124] \\
\hline
\end{tabular}


Table 2 Data collection (Continued)

\begin{tabular}{|c|c|c|c|c|c|}
\hline ID45 & SAM-V & Cand. P. ubique (N) & Ligand & Test & {$[125]$} \\
\hline ID46 & THF & Eubacterium siraeum (P) & Ligand & Train & {$[126,127]$} \\
\hline ID47 & THF & Clostridium kluyveri (P) & Ligand & Test & {$[126]$} \\
\hline ID48 & TPP & Escherichia coli $(\mathrm{N})$ & Ligand & Train & {$[30,128-130]$} \\
\hline ID49 & TPP & Bacillus subtilis (P) & Ligand & Test & {$[26,129]$} \\
\hline ID50 & Tryptophan & Escherichia coli (N) & Complex & Train & {$[8,131]$} \\
\hline ID51 & Tryptophan & Bacillus subtilis $(P)$ & Complex & Test & {$[132,133]$} \\
\hline \multirow[t]{2}{*}{ ID52 } & Tuco & Geobacter metallireducens (N) & Ligand & Test & {$[111]$} \\
\hline & $y \times k D$ & Bacillus subtilis $(P)$ & Motif & None & [76] \\
\hline
\end{tabular}

Collected sequences from literature observed to have more than one secondary structure. P corresponds to gram-positive and $\mathrm{N}$ corresponds to gram-negative. Genomic locations are available in Table 3.

${ }^{[1]}$ Table 2: This sequence overlaps codons. $\mathrm{pH}$ also has a role in alteration of structure.

${ }^{[2]}$ Table 2: Downstream-peptide.

some other ribo-regulators, although we refer to all these sequences as riboswitches, here. A subset of such sequences were selected as the positive control set of sequences having two structures. The criterion for selecting such a subset was minimum length of the RNA that exhibits alternative folds for each sequence. This criterion is further explained in Methods. The resulting set of length variant sequences are described in Tables 2 and 3.

\section{Mutagenesis}

To investigate the relationship of various structural features to the folding space of the riboswitches, we compared their wild-type value to that of structural and non-structural mutants. By structural mutants, we mean those mutant sequences that were designed to disrupt either of the two biologically functional conformations of the riboswitch. These structural mutants, whose regulatory functions had been experimentally investigated, were gathered from the literature. These mutant sequences may not have been naturally occurring biological sequences. Nevertheless, having very similar sequence features to their wild type, this enables us to evaluate the variations of structural features with respect to loss of functionality given closest possible negative controls. The percentage of change in feature values for mutants relative to the wild type is shown in Table 4. If there is a relationship between the features and alternative folds, one would expect the values corresponding to structural mutants (denoted as YES) to be significantly less than that of the wild type and non-structural mutants (denoted as NO). A simplified criterion to calculate the performance of each feature was to define true positives as negative values in structural mutants and true negatives as zero or positive values in non-structural mutants. Hence, we calculated sensitivity and specificity of each feature to structural mutants under the above criterion. Here, Sensitivity and Specificity symbolize the performance of a classifier that, based on the diversity value of the wild type and a non-functional mutant, predicts if the mutant is a structural mutant (denoted YES) or not. The classifier rule here is that structural mutants must have a lower value. Performance of each feature is shown in Table 5. The performance of the base-pairing entropy BJKbp is higher than other features on average. This suggests that structural mutants are expected to have lower base-pairing entropy than non-structural mutants and wild type 83.33 percent of the times, while non-structural mutants are expected to equal or higher values than the while type 83.33 of the times. The performance of the structural entropy under the same folding model $B J K$ was slightly lower, while being higher than those for the $R N D$ and Sil features. Features BJKbp, BJK, and RND corresponding to the B. subtilis Magnesium 
Table 3 Genomic locations of collected sequences

\begin{tabular}{|c|c|c|c|c|c|}
\hline ID & Accession & Start & End & Strand & Length \\
\hline ID01 & U00096.3 & 3442440 & 3442547 & - & 108 \\
\hline ID02 & NC_000964.3 & 486099 & 486230 & + & 132 \\
\hline ID03 & AE017180.2 & 2773395 & 2773492 & + & 98 \\
\hline ID04 & СР000860.1 & 1860063 & 1860186 & - & 124 \\
\hline ID05 & U00096.3 & 4163564 & 4163632 & + & 69 \\
\hline ID06 & BA000040.2 & 5279368 & 5279482 & - & 115 \\
\hline ID07 & AE006468.1 & 2113803 & 2113897 & - & 95 \\
\hline ID08 & CP000075.1 & 1675079 & 1675157 & - & 79 \\
\hline ID09 & СР000702.1 & 1794825 & 1794895 & + & 71 \\
\hline ID10 & AE017194.1 & 4815592 & 4815665 & + & 74 \\
\hline ID11 & AE009951.2 & 2496 & 2668 & + & 173 \\
\hline ID12 & U00096.3 & 3184455 & 3184718 & - & 264 \\
\hline ID13 & NC_000964.3 & 2431380 & 2431615 & - & 236 \\
\hline ID14 & AE009951.2 & 963901 & 963988 & - & 89 \\
\hline ID15 & NC_000964.3 & 2549381 & 2549501 & - & 121 \\
\hline ID16 & AE000512.1 & 1519015 & 1519250 & - & 236 \\
\hline ID17 & NC_000964.3 & 2910878 & 2911045 & - & 170 \\
\hline ID18 & СР001363.1 & 4712312 & 4712483 & + & 172 \\
\hline ID19 & U00096.3 & 4467416 & 4467525 & + & 110 \\
\hline ID20 & NC_000964.3 & 1395622 & 1395825 & + & 204 \\
\hline ID21 & U00096.3 & 816923 & 817041 & + & 119 \\
\hline ID22 & U00096.3 & 3238486 & 3238569 & + & 84 \\
\hline ID23 & СР003959.1 & 4635235 & 4635309 & + & 75 \\
\hline ID24 & AE007317.1 & 904178 & 904257 & + & 80 \\
\hline ID25 & NC_000964.3 & 1439279 & 1439338 & + & 60 \\
\hline ID26 & AE016796.2 & 504379 & 504491 & + & 113 \\
\hline ID27 & NC_000964.3 & 626329 & 626426 & - & 98 \\
\hline ID28 & NC_000964.3 & 2320055 & 2320196 & - & 142 \\
\hline ID29 & U55047.1 & 3107 & 3215 & + & 109 \\
\hline ID30 & U00096.3 & 3867416 & 3867488 & - & 73 \\
\hline ID31 & BA000012.4 & 1943727 & 1943820 & - & 94 \\
\hline ID32 & AY316747.1 & 197909 & 198004 & + & 96 \\
\hline ID33 & AP012279.1 & 5017601 & 5017677 & - & 135 \\
\hline ID34 & AL646052.1 & 1348529 & 1348625 & + & 97 \\
\hline ID35 & AE008691.1 & 1750249 & 1750372 & - & 124 \\
\hline ID36 & NC_000964.3 & 1180646 & 1180802 & - & 157 \\
\hline ID37 & AE007869.2 & 2703460 & 2703559 & + & 100 \\
\hline ID38 & CP000725.1 & 1038292 & 1038371 & + & 80 \\
\hline ID39 & СР003726.1 & 618415 & 618496 & + & 82 \\
\hline ID40 & NC_003888.3 & 2308634 & 2308770 & - & 137 \\
\hline ID41 & AE000516.2 & 3723565 & 3723713 & + & 149 \\
\hline ID42 & AAYC01000001.1 & 142052 & 142099 & + & 48 \\
\hline ID43 & ABID01000011.1 & 17036 & 17084 & - & 49 \\
\hline ID44 & СР000084.1 & 1005827 & 1005879 & + & 53 \\
\hline ID45 & СР000084.1 & 1127359 & 1127423 & - & 65 \\
\hline ID46 & FP929059.1 & 95139 & 95281 & - & 144 \\
\hline ID47 & NC_009706.1 & 3903929 & 3904072 & + & 144 \\
\hline ID48 & U00096.3 & 2185279 & 2185426 & - & 148 \\
\hline ID49 & NC_000964.3 & 1242265 & 1242422 & + & 158 \\
\hline ID50 & U00096.3 & 1322975 & 1323055 & - & 81 \\
\hline ID51 & NC_000964.3 & 2377419 & 2377559 & - & 141 \\
\hline ID52 & СР000148.1 & 1157816 & 1157926 & - & 111 \\
\hline
\end{tabular}

Column ID corresponds riboswitches in Table 2. 
structural mutants M5 and M6 were all positive, implying that our hypothesis of higher structural entropy and alternative fold does not hold for this riboswitch. The average silhouette index of energy landscapes ( $\mathrm{Sil}$ ) has a much better performance for the mentioned riboswitches. This could either be because SCFG models fail to capture conformational dynamics of this riboswitch or the thermodynamic equilibrium between its alternative folds is more subtle than expected.

\section{Sense-antisense classification results}

Classification of the RNA sequence into riboswitches and antisense sequences was done using binomial logistic regression. Sequence features, such as Length $L$, Minimum Free Energy $M F E$, GC-composition $G C$, and structural entropy were used for classification. The MFE value was included as a relative measure of structural stability. An initial investigation of the power of selected features in sense-antisense discrimination was done through cross-validation for all 104 (52 riboswitches and 52 antisense) sequences. Binomial logistic regression classification probabilities were assigned to each sequence based on the other 104 sense and antisense sequences. It is shown in Table 6 that features $\{L, G C, G U, S i l\}$ result in the highest true positive rate, lowest false positive rate, and highest area under the receiver operating characteristic (ROC) curve. This result suggests that the folding space of the riboswitch sequence is expected to be different than that of its antisense, since the $\mathrm{Sil}$ feature is based on the clustering of the energy landscape, although further investigation into this assumption is needed.

The performance of classifiers that involved uracil composition were more dependent on sequence features rather that structure and subsequently more prone to data fitting. The reason is that uracil composition can be different the sense and antisense. Excluding classifiers that incorporate uracil composition (i. e. forth set of rows in Table 6) showed that the features sets $\{L, M F E, G C, R N D\}$ and $\{L, M F E, G C, B J K\}$ had a fairly acceptable performance. The performance of the corresponding feature sets were higher than the $\{L, M F E, G C\}$ classifier. Furthermore, inclusion of uracil composition into the classifier lowered performance (See $\{L, M F E, G C, U\}$ in Figure 2). It is noteworthy to recall that the above classifiers neither represent the most informative features of the data nor are they tuned for best performance (Please refer to Methods section for details on calculating performance). Therefore, structural entropy may be informative in sense-antisense classification since $L$ and $G C$ are equal for each pair of sense and antisense. The performance of $\{L, M F E, G C, B J K b p\}$ was also higher than $\{L, M F E, G C\}$ but slightly lower than feature sets that incorporate structural entropy values. The ROC curve corresponding to these classifiers is shown in Figure 2.

The sense-antisense results show that the above approach has a high false positive rate, should it be used for riboswitch discovery. It also does not fully address all questions about its performance. For instance, is structural entropy orthogonal to results of other available methods that aim to capture conformational switches, or is it highly correlated with them? What is the significance of incorporating features $L, G C$, and $M F E$ in the classifiers? How much of the performance of the above classifiers, such as $\{L, G C, G U, S i l\}$, reflect structural characteristics of the riboswitches and how much is due to other features of the data? How generalizable are the results and what is the performance of classification when tested on riboswitches from distant organisms with very different genomic compositions? Can we distinguish riboswitches from their antisense and other similar sequences with 
Table 4 Mutagenesis

\begin{tabular}{|c|c|c|c|c|c|c|}
\hline $\begin{array}{l}\text { Wild-type } \\
\text { ID49 }\end{array}$ & $\begin{array}{l}\text { Riboswitch (Length) } \\
\text { TPP (158) }\end{array}$ & \multicolumn{2}{|l|}{ Organism } & $\begin{array}{l}\text { Sensitivity \% } \\
56.9\end{array}$ & \multicolumn{2}{|l|}{ Specificity \% } \\
\hline Mutants [26] & Function & Disruption of only one structure & $\triangle \mathrm{RND} \%$ & $\Delta \mathrm{BJK} \%$ & $\Delta \mathrm{BJKbp} \%$ & $\Delta$ Sil \% \\
\hline+30 & Disrupts anti-antiterminator & Yes & 0.7 & -2.6 & -3.9 & -55.2 \\
\hline+118 & Disrupts anti-terminator & Yes & -0.4 & 5.3 & -0.7 & -50.3 \\
\hline+80 & Disrupts thi-box & No & 0.8 & 3.3 & 0.8 & -38.2 \\
\hline+97 & Disrupts thi-box & No & -0.8 & 1.9 & 1.6 & -63.2 \\
\hline Wild-type & Riboswitch (Length) & Organism & & Sensitivity \% & Specificity \% & \\
\hline ID13 & FMN (236) & B. subtilis & & $81.8^{[1]}$ & 64.3 & \\
\hline Mutants [26] & Function & Disruption of only one structure & $\Delta \mathrm{RND} \%$ & $\Delta \mathrm{BJKK} \%$ & $\Delta \mathrm{BJKbp} \%$ & $\Delta \mathrm{Sil} \%$ \\
\hline G34C/G35C & Disrupts anti-terminator & Yes & -1.6 & -5.5 & -2.4 & 15.4 \\
\hline $\mathrm{C} 86 \mathrm{~T}$ & Disrupts rfn-box & No & 0.2 & -0.1 & 0.6 & 11.8 \\
\hline C49T & Disrupts rfn-box & No & 0.3 & 0.5 & 0 & -14.3 \\
\hline G157A/G160A & Disrupts anti-antiterminator & Yes & 0 & -0.7 & -0.9 & 66.7 \\
\hline Wild-type & Riboswitch (Length) & Organism & & Sensitivity \% & Specificity \% & \\
\hline ID36. ${ }^{[2]}$ & SAM-I (159) & B. subtilis & & 94 & $\mathbf{8 8 . 7}$ & \\
\hline Mutants [134] & Function & Disruption of only one structure & $\Delta \mathrm{RND} \%$ & $\Delta \mathrm{BJK} \%$ & $\Delta \mathrm{BJKbp} \%$ & $\Delta$ Sil \% \\
\hline Ma & Disturbs both structures & No & 2.3 & 15.8 & 10.7 & -48.8 \\
\hline Mab & Disrupts anti-terminator & Yes & -2.3 & -0.29 & -0.4 & 4.1 \\
\hline Mc & Disrupts anti-terminator & Yes & 0.3 & -0.31 & -0.8 & -0.3 \\
\hline Mabc & Compensates mutations to wild type & No & -1.1 & -0.32 & -0.7 & -3.2 \\
\hline Wild-type & Riboswitch (Length) & Organism & Reference & Sensitivity \% & Specificity \% & \\
\hline ID18 & Magnesium (172) & Salmonella enterica & & $64.5^{[3]}$ & 43.5 & \\
\hline Mutant [20] & Function & Disruption of only one structure & $\triangle \mathrm{RND} \%$ & $\Delta \mathrm{BJJK} \%$ & $\Delta \mathrm{BJKbp} \%$ & $\Delta \mathrm{Sil} \%$ \\
\hline C145G & Favors high $\mathrm{Mg}^{+}$conformation & Yes & 1.7 & -1.8 & -4.7 & -10.1 \\
\hline
\end{tabular}


Table 4 Mutagenesis (Continued)

\begin{tabular}{|c|c|c|c|c|c|c|}
\hline Wild-type & Riboswitch (Length) & Organism & & Sensitivity \% & Specificity \% & \\
\hline ID12 & FMN (264) & E. coli & & 38.9 & 32.3 & \\
\hline Mutants [20] & Function & Disruption of only one structure & $\triangle \mathrm{RND} \%$ & $\Delta \mathrm{BJK} \%$ & $\Delta \mathrm{BJKbp} \%$ & $\Delta \mathrm{Sil} \%$ \\
\hline M1 & Favors +FMN conformation & Yes & 0.4 & -3.8 & -5.8 & -43 \\
\hline M2 & Favors -FMN conformation & Yes & -1.4 & -1.9 & -1.2 & -5.7 \\
\hline Wild-type & Riboswitch (Length) & Organism & & Sensitivity \% & Specificity \% & \\
\hline ID20 & Magnesium (204) & B. subtilis & & 78 & 65 & \\
\hline Mutants [19] & Function & Disruption of only one structure & $\triangle \mathrm{RND} \%$ & $\Delta \mathrm{BJK} \%$ & $\Delta \mathrm{BJKbp} \%$ & $\Delta \mathrm{Sil} \%$ \\
\hline M5 & Disrupts termination & Yes & 2.7 & 0.9 & 0.7 & -12.3 \\
\hline M6 & Distrupts anti-terminator & Yes & 3.9 & 12.4 & 8 & -14.8 \\
\hline Wild-type & Riboswitch (Length) & Organism & & Sensitivity $\%$ & Specificity \% & \\
\hline ID33 & ROSE-P2 (135) & Bradyrhizobium & & $22.7^{[4]}$ & 22.2 & \\
\hline Mutant [22] & Function & Disruption of only one structure & $\triangle \mathrm{RND} \%$ & $\Delta \mathrm{BJK} \%$ & $\Delta \mathrm{BJKbp} \%$ & $\Delta \mathrm{Sil} \%$ \\
\hline$\Delta \mathrm{G} 3^{[5]}$ & Deletion of a critical nucleotide & Yes & -2.6 & -8.1 & -4.7 & 8.6 \\
\hline
\end{tabular}

Percentage of change in entropy values of mutants compared to wild type. Mutation names are according to the literature. Type of disruption to wild type activity/conformation is denoted in column funct ion (please see references for more detail on mutation information). Mutants have same length as the wild type, except for the ROSE-P2 thermosensor. Wild-type segments are the same as gathered data, except for the SAM-I riboswitch where a homologue has been used. $\triangle \mathrm{RND} \%$ and $\triangle \mathrm{BJK} \%$, refer to structural entropy values for the RND and BJK models, respectively. $\Delta \mathrm{BJKbp} \%$ refers to the base-pairing entropy of the BJK model as defined by [54]. $\Delta$ Sil\% refers to the two-cluster average silhouette index of the energy landscape of the RNA as calculated by [46]. Sensitivity\% and specificity\% refer to BJK model accuracy to the secondary structural conformation, with disregard to pseudoknots.

Wo out of the 55 base-pairings of the B. subtilis FMN sequence are G-A pairs.

${ }^{[2]}$ Table 4: ID36.1 is the met/ SAM-I riboswitch in B. subtilis and has sequence identity of $76 \%$ with ID36 yitJ B. subtilis SAM-I riboswitch using BLASTO. Sequence location on Location on the B. subtilis str. 168 strain embAL009126.3 (1258304-1258462), forward strand.

${ }^{33}$ Table 4: CYK structural prediction under the BJK model and that of the MFE model via vienna@detect different alteration of the Magnesium riboswitch in Salmonella enterica serovar Typhimurium. Structural distance of the MFE prediction to the high Mg2 ${ }^{+}$and low Mg2 ${ }^{+}$structures are 28 and 120, respectively while they are 114 and 74, under CYK-based structural prediction of the BJK model. Sensitivity and specificity values for the BJK model prediction of the low $\mathrm{Mg}^{+}$conformation are $22 \%$ and $22 \%$.

${ }^{[4}$ Table 4: One out of the 44 base-pairngs of the Bradyrhizobium ROSE-P2 sequence is a G-G pair.

${ }^{[5]}$ Table 4: The $\Delta G 83$ mutant is one nucleotide shorter than the ROSE-P2 135 nt-long wild type. 
Table 5 Mutagenesis results

\begin{tabular}{lccc}
\hline Feature & Sensitivity (\%) & Specificity (\%) & MCC \\
\hline BJKbp & 83.33 & 83.33 & 0.645 \\
BJK & 75 & 66.67 & 0.403 \\
RND & 41.67 & 66.67 & 0.08 \\
SIL & 66.67 & 16.67 & -0.175
\end{tabular}

Classification rule: Lower value than wild type predicts structural mutant, while higher or equal value predicts non-structural mutants. Positive control: Structural Mutants of a given wild type. Negative control: non-structural mutants of a given wild type. See the Methods section for details on calculating sensitivity and specificity.

no structure, simultaneously? Does the structural entropy of a typical riboswitch tend to be lower or higher than that of its antisense sequence? To address the above questions, first we calculated the correlation between structural entropy features and other results from gathered tools. For this purpose, we performed the correlations for all riboswitches and their antisense sequences, totalling 104 sequences. Correlation values between structural entropy and other conformational features are illustrated in Table 7 for both folding models, RND and BJK, along with correlation values corresponding to structural entropy normalized to sequence length. By inspection, we can see that structural entropy is not

Table 6 Classification performance using cross validation

\begin{tabular}{|c|c|c|c|c|}
\hline Classifier & TP rate & FP rate & MCC & R.O.C. area \\
\hline$\{L, G C, G \cup, S i\}$ & 0.750 & 0.250 & 0.500 & 0.826 \\
\hline$\{L, G C, G U\}$ & 0.673 & 0.327 & 0.346 & 0.700 \\
\hline$\{L, G C, G \cup, B J K\}$ & 0.644 & 0.356 & 0.289 & 0.691 \\
\hline$\{L, G C, G \cup, B J K b p\}$ & 0.654 & 0.346 & 0.309 & 0.690 \\
\hline$\{L, G C, G \cup, R N D\}$ & 0.654 & 0.346 & 0.308 & 0.689 \\
\hline$\{L, M F E, G C, G U, R N D\}$ & 0.673 & 0.327 & 0.346 & 0.714 \\
\hline$\{L, M F E, G C, G \cup\}$ & 0.654 & 0.346 & 0.308 & 0.707 \\
\hline$\{L, M F E, G C, G \cup, B J K\}$ & 0.663 & 0.337 & 0.327 & 0.703 \\
\hline$\{L, M F E, G C, G \cup, B J K b p\}$ & 0.663 & 0.337 & 0.327 & 0.701 \\
\hline$\{L, M F E, G C, G U, S i l\}$ & 0.625 & 0.375 & 0.250 & 0.697 \\
\hline$\{L, M F E, G U\}$ & 0.663 & 0.337 & 0.327 & 0.710 \\
\hline$\{L, M F E, G U, R N D\}$ & 0.663 & 0.337 & 0.327 & 0.702 \\
\hline$\{L, M F E, G U, B J K b p\}$ & 0.663 & 0.337 & 0.32 & 0.701 \\
\hline$\{L, M F E, G U, S i\}$ & 0.654 & 0.346 & 0.308 & 0.701 \\
\hline$\{L, M F E, G U, B J K\}$ & 0.644 & 0.356 & 0.289 & 0.699 \\
\hline$\{L, M F E, G C, R N D\}$ & 0.663 & 0.337 & 0.327 & 0.708 \\
\hline$\{L, M F E, G C, B J K\}$ & 0.663 & 0.337 & 0.327 & 0.703 \\
\hline$\{L, M F E, G C, B J K b p\}$ & 0.635 & 0.365 & 0.269 & 0.683 \\
\hline$\{L, M F E, G C\}$ & 0.606 & 0.394 & 0.212 & 0.650 \\
\hline$\{L, M F E, G C, S i\}$ & 0.635 & 0.365 & 0.270 & 0.644 \\
\hline$\{L, M F E, G C U, R N D\}$ & 0.644 & 0.356 & 0.289 & 0.693 \\
\hline$\{L, M F E, G C U, B J K\}$ & 0.625 & 0.375 & 0.250 & 0.617 \\
\hline$\{L, M F E, G C U, B J K b p\}$ & 0.596 & 0.404 & 0.193 & 0.595 \\
\hline$\{L, M F E, G C U\}$ & 0.587 & 0.413 & 0.174 & 0.581 \\
\hline$\{L, M F E, G C U, S i l\}$ & 0.548 & 0.452 & 0.097 & 0.554 \\
\hline
\end{tabular}

104-fold binomial logistic classifiers on all of the 52 riboswitch sequences and their antisense sequences. classifier features shown in legend. Weka@open source software package used. Features $L, M F E, G C, G U, G C U$ and $U$ denote length, MFE,

GC-composition, and uracil frequency, respectively. Features $R N D$ and $B J K$ denote structural entropy of the RND and BJK models, respectively. as defined in [54]. Feature BJKbp denotes base-pairing entropy as defined in [54]. Feature Sil denotes the two-cluster average Silhouette index of energy landscape as calculated in [46]. 


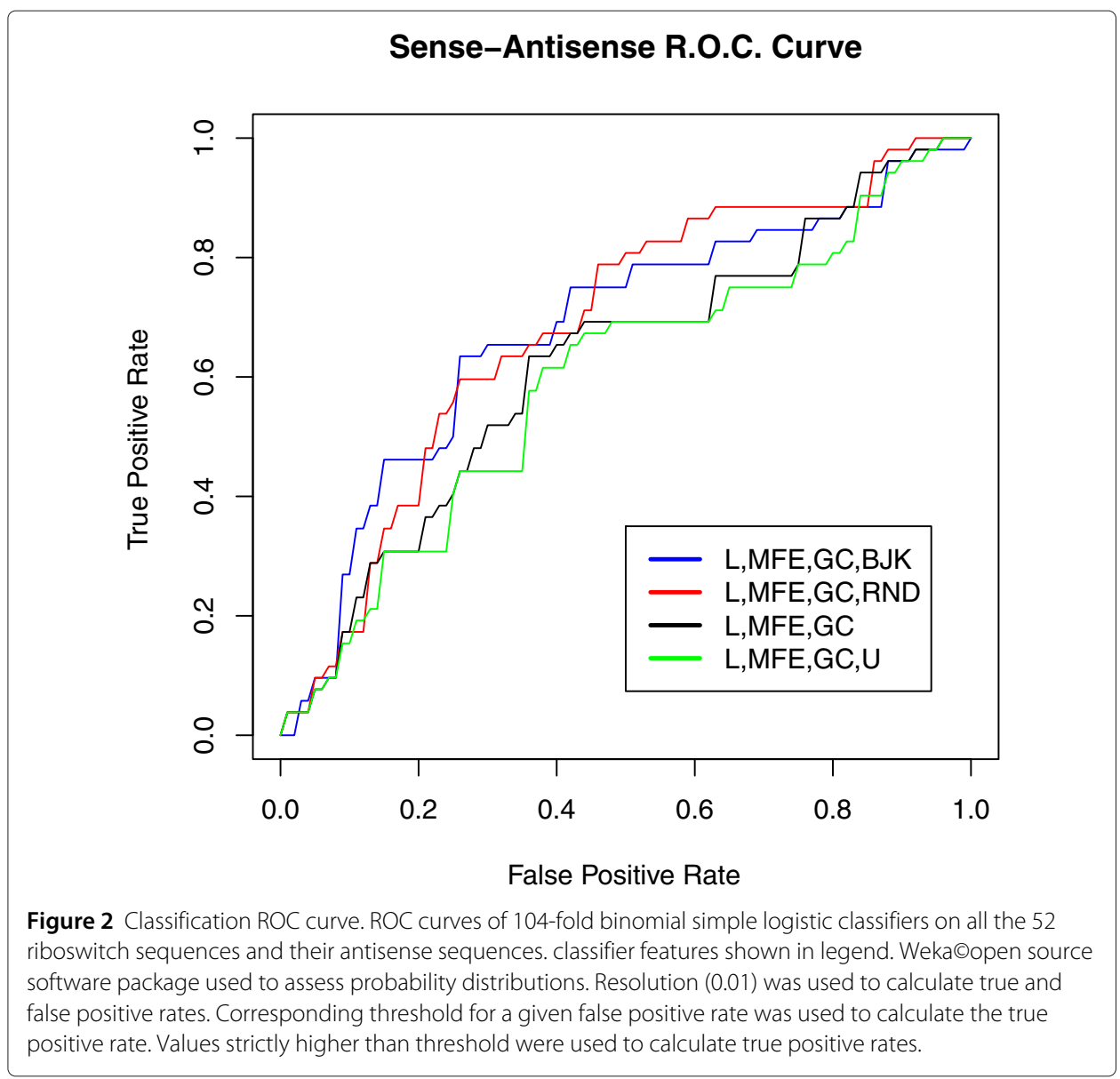

necessarily highly correlated with other features, suggesting the possibility that it may contain additional information about RNA sequences, in general. We neither rigorously calculated the significance of correlation values nor did we further evaluate the orthogonality of structural entropy to other features. We then selected classifiers $\{L, G C, G U, S i l\}$, $\{L, M F E, G C, R N D\},\{L, M F E, G C, B J K\}$, and $\{L, M F E, G C\}$ for further investigation. They are referred to as LGCGUSIL, LMFEGCRND, LMFEGCBJK, and LMFEGC. We divided our data into training and test sets, each having different average GC-composition. Sequence segments and their corresponding structures are included in sections Training set and Test set in Appendix. We then evaluated the performance of the classifiers from the training set using the test set.

Table 7 Correlations between entropy values and other approaches

\begin{tabular}{lccc}
\hline Correlations & FFTbor & RNAShapes & Sil \\
\hline RND & -0.46 & -0.12 & -0.42 \\
BJK & -0.5 & -0.18 & -0.46 \\
RND Normalized & -0.29 & -0.15 & -0.34 \\
BJK Normalized & -0.34 & -0.36 & -0.41 \\
\hline
\end{tabular}

RND and BJK are the structural entropy values for corresponding models. Normalized RND or BJK are corresponding values normalized to sequence length. FFTbor, RNAShapes, and Sil are other conformation features calculated according to Methods section. 


\section{Random shuffles}

First, in order to assess the relationship between the performance of the above binomiallogistic-regression classifiers to structural features, we performed dinucleotide shuffling test [60]. We originally generated 10 dinucleotide shuffles for each of the riboswitches in the training and test sets. We used riboswitches of the training set and their corresponding random shuffles to estimate binomial logistic-regression coefficients. We then used the coefficients to classify sequences of the test set and their corresponding random shuffles. However, the classification performance and corresponding ROC curves were highly dependent on the MFE feature (data not shown). In order to both have a better insight into the structural entropy feature and a more clear comparison to other methods, we then filtered both the random shuffles of the training and test sets to have similar MFE values to their corresponding riboswitches and repeated the test (please see Methods section and Table 8 for information on the filtered dinucleotide shuffles). The ROC curves and performance values can be seen in Figure 3 and Table 9, respectively. The classifier LGCGUSIL poorly distinguishes riboswitches from random shuffles compared to the other classifiers. This suggests that the high performance of this classifier in sense-antisense classification was not necessarily due to structural features. Although the RNAShapes classifier has higher performance as shown in Table 8, it only corresponds to one point of the ROC curve. In order to have a more comprehensive measure of performance, we calculated the area under the ROC curve for the classifiers. The performance of most classifiers is roughly similar with the LMFEGCRND having the second highest value after LMFEGC (see Figure 3 and area under ROC curve in the legend). Although filtering only for those dinucleotide shuffles having similar MFE would seem a reasonable negative control, we did not further investigate random shuffle test. Preparing a random-shuffle negative control ensemble of sequences with similar length, composition, and MFE values that homogeneously represent all riboswitches may be a formidable task and not necessarily helpful with evaluating our approach that focuses on real biological sequences as negative control.

To evaluate the significance of feature $L$, we considered both constant choice of length and variable choice of length for riboswitches. Apart from the antisense sequence, untranslated regions (UTR) shorter than 80 nt have been selected as another negative set, since they are unlikely to contain structures over such a short length. Some riboswitch sequence segments, however, were selected to be shorter than this length. The length of the corresponding UTR (from transcription binding site to the translation start codon) for riboswitches, however, were not shorter than $80 \mathrm{nt}$. UTRs corresponding to the $\sigma-70$ in $E$. coli with distance less than $80 \mathrm{nt}$ from the translation start codon were used here as

Table 8 Average and standard deviation values of Length, MFE, and GC-compositions of the training and test sets along with their filtered dinucleotides shuffles

\begin{tabular}{ccccccc}
\hline Train & L & MFE & GC & std(L) & std(MFE) & std(GC) \\
\hline Riboswitch & 114.10 & -41.05 & 0.52 & 49.27 & 23.83 & 0.10 \\
Shuffles & 127.0 & -38.19 & 0.55 & 47.85 & 18.51 & 0.09 \\
\hline Test & $\mathbf{L}$ & MFE & GC & std(L) & std(MFE) & std(GC) \\
\hline Riboswitch & 120.74 & -44.63 & 0.49 & 46.71 & 18.60 & 0.09 \\
Shuffles & 141.77 & -40.09 & 0.51 & 38.97 & 11.97 & 0.08 \\
\hline
\end{tabular}

Column Riboswitch denotes riboswitches. Column Shuffles denotes filtered dinucleotide shuffles. Total number of filtered dinucleotide shuffles are 216 and 151, in the training and testing sets, respectively. 


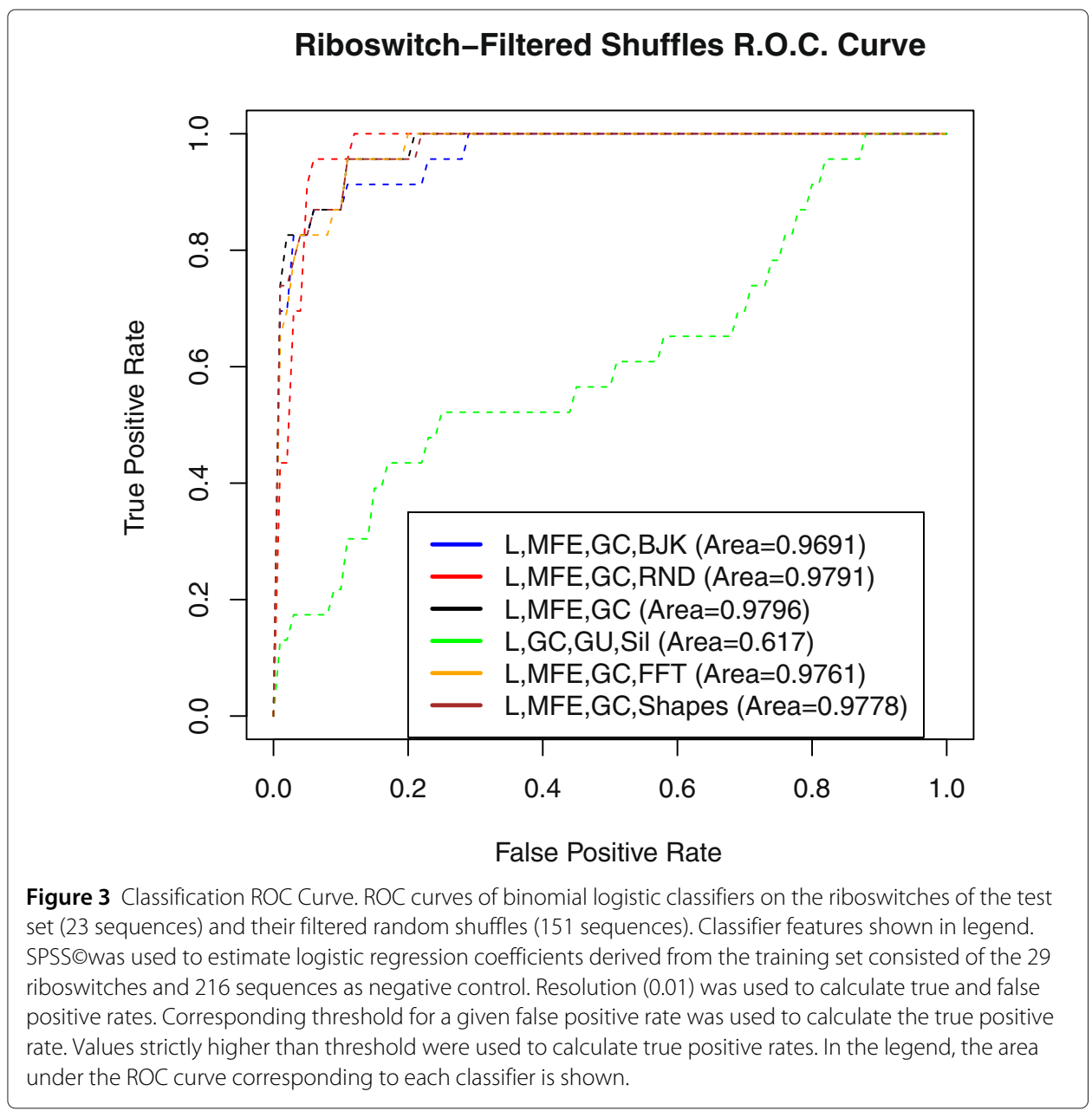

sequences that do not contain structure. 30 sequence segments were selected from $\sigma-70$ E. coli UTRs shorter than $80 \mathrm{nt}$ (see Table 10 for information on sequence locations). The section Methods extensively discusses the criteria for selecting the subsets, dividing the riboswitches and E. coli UTRs into training/test sets, as well as information on data sets. Average and standard deviation of features $L, M F E$, and $G C$ for the training and test sets are shown in Table 11. The free energy of the centroid structure [35] calculated by CentroidFold $\odot$ [62], denoted here as CFE, was also used as a substitute for MFE.

Table 9 Classification performance in dinucleotide shuffling test

\begin{tabular}{lcc}
\hline Classifier & TP\% & FP\% \\
\hline LMFEGCBJKbp & 65.2 & 0.7 \\
LMFEGCBJK & 69.6 & 0.7 \\
LMFEGCRND & 69.6 & 3.3 \\
LMFEGC & 69.6 & 0.7 \\
LGCGUSIL & 0.0 & 0.0 \\
LMFEGCFFT & 65.2 & 1.3 \\
LMFEGCShapes & 73.9 & 0.7
\end{tabular}

Actual length of sequences used in this test. Column Features denotes features used from the training set. TP\% denotes percentage of true positives. FP\% represent the percentages of dinucleotide shuffles that are misclassified as riboswitches. Please see Methods section for details on preparing dinucleotide shuffles. 
Table 10 Short UTR collection

\begin{tabular}{|c|c|c|c|c|}
\hline Start & End & Strand & Gene & Length \\
\hline 42325 & 42403 & + & fixA & 79 \\
\hline 246641 & 246712 & + & yafL & 72 \\
\hline 570070 & 570116 & + & $y b c L$ & 47 \\
\hline 848134 & 848173 & - & $d p s$ & 40 \\
\hline 879876 & 879950 & + & dacC & 75 \\
\hline 989579 & 989637 & - & $p \cap c B$ & 59 \\
\hline 1108480 & 1108558 & + & mdoG & 79 \\
\hline 1331812 & 1331879 & + & CysB & 68 \\
\hline 1397550 & 1397576 & - & fnr & 27 \\
\hline 1570069 & 1570096 & - & $\operatorname{gad} B$ & 28 \\
\hline 1732381 & 1732459 & + & mepH & 79 \\
\hline 1927731 & 1927756 & - & yebE & 26 \\
\hline 2039370 & 2039399 & + & $\operatorname{zin} T$ & 30 \\
\hline 2268700 & 2268748 & + & $r t n$ & 49 \\
\hline 2380676 & 2380735 & + & elaD & 60 \\
\hline 2541550 & 2541579 & - & cys $P$ & 30 \\
\hline 2823813 & 2823854 & + & SrlA & 42 \\
\hline 2982146 & 2982216 & - & kdul & 71 \\
\hline 3134393 & 3134425 & - & pitB & 33 \\
\hline 3276888 & 3276936 & + & $k b a z$ & 49 \\
\hline 3467875 & 3467918 & - & chiA & 44 \\
\hline 3651959 & 3651984 & + & $s / p$ & 26 \\
\hline 3735493 & 3735520 & + & mals & 28 \\
\hline 3845190 & 3845221 & - & uhpt & 32 \\
\hline 3909548 & 3909591 & - & psts & 44 \\
\hline 4028994 & 4029036 & - & $f a d B$ & 43 \\
\hline 4213425 & 4213501 & + & $a c e B$ & 77 \\
\hline 4244442 & 4244487 & - & male & 46 \\
\hline 4358054 & 4358129 & - & $c a d B$ & 76 \\
\hline 4492620 & 4492646 & + & indK & 27 \\
\hline
\end{tabular}

30 randomly chosen untranslated regions of lengths less than $80 \mathrm{nt}$ corresponding to the $\sigma-70$ transcription factor binding sites in Escherichia coli str. K-12 substr. MG1655 (GenBankOID: U00096.2). Column Start denotes start of the binding site. End denotes the downstream start codon. Gene denotes the name of the first gene in the corresponding mRNA. Length denotes the length of the UTR.

The performance of the tri-state classifier was evaluated by estimating classifier parameters from multinomial logistic regression of the training sets and then calculating the correct classification of sequences having zero (E. coli) riboswitch structure, one possible structure (antisense), or two (riboswitch) structures that are in the test set. We also evaluated the performance of classifiers that incorporate features from FFTbor and RNAShapes software packages calculated according to Methods section. Feature sets $\{L, M F E, G C, F F T b o r\}$ denoted as LMFEGCFFTbor and $\{L, M F E, G C, R N A S h a p e s\}$ denoted as LMFEGCShapes were included in the test.

Classification performance values are denoted in Table 12 along with sensitivity of each classifier. Sensitivity of tri-state classifiers were defined here as total number of correctly classified sequences divided by total number of sequences classified. Model LMFEGCBJK resulted in both highest sensitivity $(80.2 \%)$ and highest percentage of correctly classified riboswitches $(91.3 \%)$. Performance of other classifiers was in the same range. Further tests are needed to make better comparison between the performance of the classifiers. The 
Table 11 Riboswitch statistics

\begin{tabular}{ccccccc}
\hline Total & $\mathbf{L}$ & MFE & GC & std(L) & std(MFE) & std(GC) \\
\hline Sense & 117.04 & -42.63 & 0.51 & 47.81 & 21.54 & 0.09 \\
Antisense & 117.04 & -37.73 & 0.51 & 47.81 & 19.55 & 0.09 \\
UTR & 49.53 & -6.48 & 0.37 & 19.37 & 5.77 & 0.08 \\
\hline Train & $\mathbf{L}$ & MFE & GC & std(L) & std(MFE) & std(GC) \\
\hline Sense & 114.1 & -41.05 & 0.52 & 49.27 & 23.83 & 0.1 \\
Antisense & 114.1 & -37.73 & 0.52 & 49.27 & 21.32 & 0.1 \\
UTR & 48.18 & -5.4 & 0.35 & 18.27 & 5.1 & 0.08 \\
\hline Test & $\mathbf{L}$ & MFE & GC & std(L) & std(MFE) & std(GC) \\
\hline Sense & 120.74 & -44.63 & 0.49 & 46.71 & 18.6 & 0.09 \\
Antisense & 120.74 & -37.74 & 0.49 & 46.71 & 17.54 & 0.09 \\
UTR & 51.31 & -7.9 & 0.39 & 21.35 & 6.47 & 0.08 \\
\hline
\end{tabular}

Average and standard deviation values of Length, MFE, and GC-compositions of the training and test sets. Column Sense denotes riboswitches. Column UTR denotes E. coli UTR sequences collected.

low performance of the LMFEGCRND model shows that classification is potentially sensitive to features length, GC-composition, and MFE, since they are different between the training and the test sets (see Table 11). Furthermore, choice of modeling is very critical in designing sense-antisense classification. The BJK model, being a more accurate folding model leads to higher performance.

Regression coefficients of the classifiers corresponding to riboswitches are shown in Column $\beta_{2}$ of Table 13. Coefficients corresponding to MFE and structural entropy, are the second and forth values, respectively. For both the LMFEGCRND and LMFEGCBJK models, MFE coefficients are negative while structural entropy coefficients are positive (values are normalized with respect to antisense). This implies that if we input a riboswitch and its reverse complement to the regression-based classifiers, the strand with lower Minimum Free Energy and higher structural entropy is more likely to be the riboswitch. Hence, we have reason to believe that despite having a more stable structure, riboswitches tend to have higher structural entropy than expected ${ }^{\mathrm{b}}$. We find this observation significant, since they are consistent across two different folding models. 3D-plots of the MFE, GC-composition, and structural entropy values under the RND model for sequences of the training set are depicted in Figure 4. Top and bottom views of the grid-view of values normalized to sequence length roughly shows this distinction.

Table 12 Classification performance

\begin{tabular}{lccccc}
\hline Classifier & $\boldsymbol{T P} \%$ & $\boldsymbol{F P}_{\mathbf{1}} \%$ & $\boldsymbol{F P}_{\mathbf{2}} \%$ & Sensitivity & Sig. \\
\hline LMFEGCBJK & 91.3 & 43.5 & 15.4 & 72.9 & MFE \\
LMFEGC & 82.6 & 30.4 & 23.1 & 71.2 & MFE \\
LMFEGCRND & 73.9 & 30.4 & 38.5 & 64.4 & L,MFE \\
LMFEGCFFTbor & 82.6 & 30.4 & 23.1 & 71.2 & - \\
LMFEGCShapes & 87.0 & 34.8 & 23.1 & 71.2 & - \\
\hline
\end{tabular}

Classifier Performance. Actual length of sequences used. Column Classifier denotes features used from the training set. TP\% denotes percentage of true positives. $F P_{1} \%$ and $F P_{2} \%$ represent the percentages of antisense sequences and $E$. coli UTRs that are misclassified as riboswitches, respectively. Sensitivity denotes overall percentage of correctly classified sequences. Sig. denotes significant (less than 0.05 in the training set) features of the multinomial classifier. 
Table 13 Logistic regression coefficients of classifiers

\begin{tabular}{lcc}
\hline Classifier & $\boldsymbol{\beta}_{\mathbf{1}}$ & $\boldsymbol{\beta}_{\mathbf{2}}$ \\
\hline LMFEGCRND & $3.191,336, .683,-.723,-.465$ & $5.052,-.161,-.089,-7.454, .220$ \\
LMFEGCBJK & $10.597,-.203, .367,-10.856, .651$ & $5.524,-.082,-.132,-9.247, .120$ \\
LMFEGC & $3.869, .052, .525,-1.419$ & $3.373,-.025,-.068,-6.234$ \\
\hline
\end{tabular}

Regression coefficients (exponents) of the multinomial logistic regression classifier: intercept, Length, MFE, GC-composition, Entropy. Parameter vectors $\beta_{1}$ and $\beta_{2}$ denote coefficients for $E$. coli UTRs and ribswitch sense sequences for the riboswitches of the training set, respectively. Coefficients normalized with respect to those for riboswitch antisenses. i. e. antisense coeficients being 0 .

Testing the classifiers on constant lengths of sequences (for all training and test sets) did not increase performance (see Table 14 in Appendix), although the RND was significant for sequences of length $150 \mathrm{nt}$ in the training set. Constant length selection was based on extending (or shortening) the original choice of length of sequences from both 5' and 3' directions such that the center of the sequence does not change. We refer to this original choice of length as the actual length, hereon. We chose this scheme for simplicity. Other sequence selection methods may be preferred, since the alternative fold may occur on varying parts ( $5^{\prime}$ or $\left.3^{\prime}\right)$ of the riboswitch sequence, in general. Substitution of CFE feature instead of MFE feature resulted in lower performance of classifiers (comparing Tables 10, 11, 12, 13, 14 and 15 in Appendix).

\section{Association with high entropy}

Mutagenesis results suggested an association between alternative folds and higher structural entropy. Furthermore, regression approaches to estimate the structural entropy of the riboswitch with respect to various sequence and structural features such as MFE lead to higher classification performance in discriminating riboswitches from their antisense

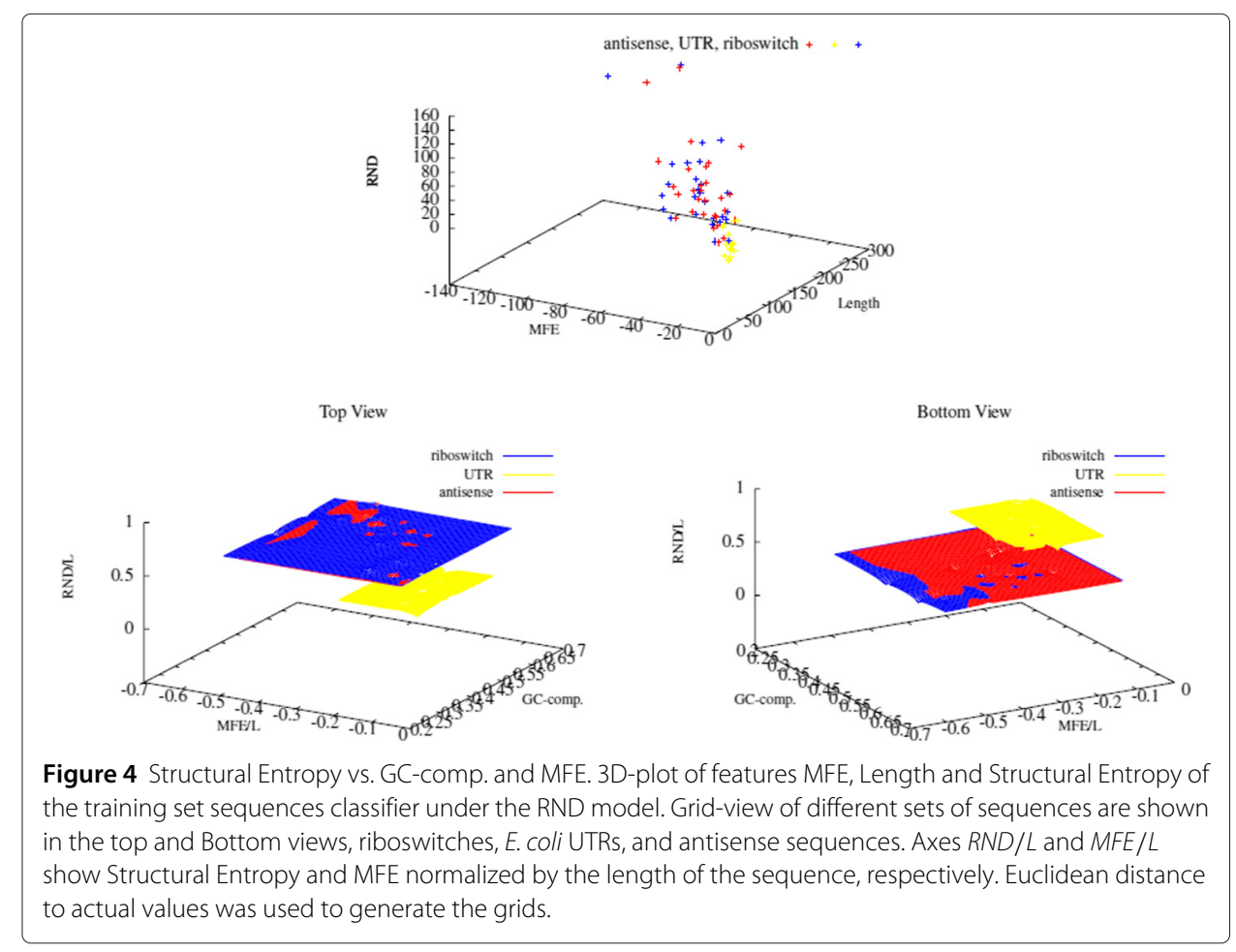


control, compared to classifiers that do not incorporate the structural entropy measure. Similar to Mutagenesis results, we observed that riboswitches tend to have higher structural entropy than what is expected of their antisense sequences. Dinucleotide shuffles test also showed an slight increase in the specificity of classifiers using one of the structural entropy features (The RND model) compared to other models and methods. We hypothesize that the structural entropy value of riboswitches may be a significant factor within the context of their length, GC-composition, and folding stability (here, MFE). In specific, conformational switches (here riboswitches) show a slight but consistent increase in their entropy values than structural mutants or antisense. The higher entropy was not, however, observed in the dinucleotide shuffling test. Under this hypothesis and the limitations of our tests from both mutagenesis and sense-antisense classification, the B. subtilis Magnesium and SAM-I riboswitches seem to be two immediate outliers.

Base-pairing entropy feature had higher performance in the Mutagenesis results, while the energy landscape index led to higher performance in sense-antise classification. Putting Mutagenesis and sense-antisense results in one perspective, however, suggests a more consistent conclusion about the structural entropy compared to the other two features.

Some of the challenges in our approach to develop riboswitch identifiers were choices of sequence segment and folding model. We found it very difficult to find a subset of sequence segments from riboswitches for our training set that had the highest structural entropy. These difficulties included but were not limited to high sensitivity of structural entropy to sequence length and location and the possible varying lengths of riboswitches that have alternative structures. We arbitrarily included varying lengths of riboswitches in our training set rather than constant length, since the performance of classifiers with constant length was either lower or similar to those with varying length. Methods based on optimization of both sequence length of riboswitches and modeling their folding dynamics may prove rewarding in this regard.

The optimum length of a sequence segment that leads to identifying riboswitches can vary from one organism to another; Constant length of 100 nt segments for $E$. coli are more suitable, while 157 nt segments lead to higher performance for $B$. subtilis riboswitches. Results about sequence segments, however, had low significance due to low number of riboswitches tested in each case. We only propose that it may be possible that riboswitches from different organisms may have different ranges of sequence lengths over which alternative structure prediction becomes significant. Optimizing search parameters on a new organism sequence is potentially a difficult task. One alternative may be evaluating the behavior of structural entropy-based classifiers on data sets that are peculiar to that organism. We have not explored this approach.

\section{Choice of model}

Classification performance of sense-antisense, genome-wide sliding window tests, and mutagenesis all suggest that the BJK folding model is more sensitive to changes in the folding space than the structurally ambiguous RND model. The classification performance of the LMFEGCBJK model both on the test set and on the B. subtilis riboswitches is high given the right sequence segment is chosen. Also, the RND model does very poorly in distinguishing the folding space of riboswitch mutants from that of their wild types. On the other hand, binomial logistic regression based classification of sense and antisense of 
all riboswitches assigns slightly higher ROC area to the classifier that deploys the RND model (see Figure 2). Furthermore, riboswitch identifiers based on the RND model are more robust in terms of sequence positioning than their BJK counterparts. The RND model only enforces Watson-Crick and G-U base-pairing and is fairly a simplistic structural model. The acceptable performance of the RND model in genome-wide approaches may be due to having less structural constraints than BJK. It may be possible that training secondary structural folding models to predict RNA secondary structures comes at the cost of loss in folding dynamics information. Overall, design of a more efficient SCFG model (possibly a heavyweight folding model) to characterize better the riboswitch folding space is another bottleneck of this approach, since modeling techniques to capture conformational features have not been developed. Current RNA structure modeling is centered around prdiction of the RNA secondary structure rather than its conformational dynamics.

\section{Genome-wide analysis}

Sequence segments predicted to have potential for alternative fold for the two B. subtilis and $E$. coli intergenic regions are presented in Genome-wide scan results. The power of regression based classifiers as riboswitch predictors is not significantly high; None of the known B. subtilis riboswitches fell in the top 100 hits in genomic scans, though certain of those hits are known regulatory elements. The lack of high performance implies sensitivity of our approach to training set genomic features. Exploring other classification schemes, such as neural networks (for instance, similar to [63]) as well as incorporating a different negative-control training data than the antisense may lead to higher performance. In order to develop an organism-specific riboswitch predictor, one may gather sequences with no structure from the target organism and deploy it as a negative control for a classifier that takes structural entropy as a feature.

Many hits fell immediately upstream of operons, which could be indicative of cisregulation. Our genome-wide scan results show dependency of genomic features such as the uracil composition. Furthermore, results presented for various genome-wide scans cannot be taken into account individually, since the above methodology is a length based method. In other words, in order to identify genomic regions with highest likelihood of having a riboswitch, it is essential to combine results of genome-wide scans under different window sizes. Such combining of results also seems organism-specific. The optimum length(s) of genome-wide window scans for the riboswitch identification can be different from one organism to another. The complexity of such a test and its corresponding rigorous statistical analyses fell outside the scope of this work. Here, we limited ourselves to few genomic scans and present a subset of regions that are riboswitch candidates in most of the genome-wide tests.

\section{The cotH gene}

The top two sequence segments predicted to be riboswitches are both upstream of $\cot H$ gene and in close proximity of one another. In fact, a $628 \mathrm{nt}$ long segment is classified to be a riboswitch (four consecutive sequence segments). The 5 ' half of this segment, $\{3717412$ nt - $3717725 \mathrm{nt}\}$, contains the top two hits which are also predicted to be riboswitches by the model LMFEGCBJK in position $\{3717098 \mathrm{nt}-3717725 \mathrm{nt}\}$ on the complementary strand of B. subtilis. Naclerio et al., 1996 [64] discuss possible regulation in the vicinity of 
$\operatorname{cotH}$ gene. They also stated that no homology to this gene was revealed in the sequences presented in the data bank at the time. They hypothesized that this gene plays an important role in the formation of the spore coat. A more recent paper [65] reports about the cotH promoter mapping $812 \mathrm{bp}$ upstream of beginning of its coding region. This region covers the top two hits we have. In fact, a 200 nt scan reveals that many consecutive segments belonging to this region have significant RND entropy values $(<0.05)$. Most interestingly, the segment with highest RND entropy value on a genome-wide level and under the 200 nt window occurs 399 nt upstream of the $\operatorname{cotH}$ gene at location \{3717398$3717597+\}$. The authors also talk about $\cot G$ and $\cot H$ genes and that they are both divergently transcribed by $\sigma-\mathrm{K}$ and a potential for extensive secondary RNA structures in this unusually long untranslated region. The $\cot G$ is located in the forward strand. There are also many hits around $2000 \mathrm{nt}$ upstream of the $\cot G$-containing operon under various sliding window tests. An interesting observation about the nucleotide composition of the top hit reveals that it uniquely contains periodic runs of 6 consecutive thymines with periodicity of 12 and 15 interchangeably. A search for similar runs of thymines was done on both strands of $B$. subtilis by relaxing periodicity from $10 \mathrm{nt}$ to $18 \mathrm{nt}$ and constraining it to having at least six consecutive runs of 6-thymines using the pattern locator software [66]. The only two hits were found on the reverse strand and overlapping with the top hit: \{3717502-3717606\} and $\{3717367-3717468\}$.

The most significant structural entropy value for the longest window size (200 nt) on the $B$. subtilis genome occurred in an unusually extensive secondary structure within that genome. It may be possible that longer RNA structures contain segments having significantly high entropy (structural entropy) on a genome-wide scale. This implies that longer RNAs could potentially have a uniquely high number of secondary structural conformations. This unusually high secondary structural diversity may be related to their regulatory role. We have not yet examined the secondary structural space of other long secondary structures in various organisms. The significantly high structural entropy feature, however, may be typical of other longer secondary strucures. In a recent study on the newly discovered class of RNAs known as long ncRNAs (lncRNA), Cloutier et al. [67] show that yeast lncRNAs are involved in the timing of gene expression. It may be possible that their proposed lncRNA-dependent quick shift of gene expression is related to a high potential for diverse secondary structural conformations.

\section{The BSU tRNA 75 operon}

The sequence segment with highest classification probability that also has signficant MFE and structural entropy values is located about $2277 \mathrm{nt}$ of the upstream region of the BSU tRNA 75 Operon. The antisense control of this segment is located in a putative transcriptional regulator. It is interesting, however, that this hit occurs upstream of a tRNA operon. A $200 \mathrm{nt}$ scan reveals more hits upstream of this operon that have significant structural entropy values some of which are closer to the tRNA operon (around $2000 \mathrm{nt}$ upstream). From locations of tRNA operon [68], it can be seen that out of the five consecutive tRNA genes with isotypes Glu, Val, Thr, Tyr, and Gln, the Thr operon has attenuation [69]. Although the long distance from the downstream translation start codon does not make this a reliable riboswitch prediction, the significance of hits in the intergenic region upstream of the Thr gene and the fact that the other top hit in our classification approach was located in a long RNA, suggest the possibility that there may be a long regulatory 
RNA residing upstream of the mentioned tRNA operon, raising the interesting possibility of a putative riboswitch regulating an attenuation mechanism. Another possiblity is the existence of an open reading frame downstream of the hit. We applied GeneMark [70] and GLIMMER [71,72] gene prediction programs on the $2000 \mathrm{nt}$ long downstream sequence of this hit. The Bacillus anthracis gene model was used in GeneMark, since B. subtilis was not available. Both programs had similar gene prediction results, showing the possibility of three open reading frames on the complementary strand, with the closest one ending $46 \mathrm{nt}$ downstream of the hit.

lysP

One of the most significant hits in our classification under the $157 \mathrm{nt}$ scan occurs immediately upstream of the lysP gene. The segment corresponding to this location also has the most significant (highest) RND entropy value while having significantly low MFE $(\mathrm{p}$-Val. $<0.05)$ on a genome-wide level. This is also true for the $150 \mathrm{nt}$ window scan. Furthermore, the $200 \mathrm{nt}$ scan assigns significantly high structural entropy (RND p-Val. $<0.05)$ as well as classification probability of higher than 0.8 for this location. The 150 nt-long segment is located at \{3421066-3421215 - $\}$ between the lysine permease and BSU MISC RNA 54. Other adjacent hits that overlap BSU MISC RNA 54 do not have such high structural entropy or classification probability. It may be possible that this segment plays a crucial role in regulating the downstream gene.

\section{Cross-organism riboswitch candidates}

The B. subtilis (BSU16090) had a homologue in the E. coli operon: (sdhA, sdhB, sdhC, $\operatorname{sdhD}$, sucA, sucB, sucC, sucD), that contains the sucC as its last gene, with e-value 3e141 (see Methods section for details). The genes were associated with top hits on their upstream in B. subtilis and E. coli with probabilities 0.897 and 0.905 , respectively. Also, the $B$. subtilis sucA (BSU19370) gene had a homologue in the $E$. coli fixA,fixB,fixC,fix operon with e-value 1e-18, both of which were associated with high hits. Other matches were the B. subtilis tagA gene and the E. coli (rfe- wzzE- rffEDGHCA- wzxE- rffT- wzyErffM) operon (e-value 1e-23), the B. subtilis citB and the $E$. coli acnA genes (e-value 0 ), the $B$. subtilis cspB and the E. coli cspG genes (e-value 2e-17), and the B. subtilis ydaB gene and the E. coli caiA,caiB,caiC,caiD,caiE,caiT operon (e-value 3e-27). None of the above B. subtilis genes, however, had homologs in S. elongates that was associated with top hits in that organism, except for the B. subtilis ydaB gene. The ydaB gene had a homologue in S. elongates syc0203_c (e-value 7e-13) where the associated upstream region contained a hit with probability 0.714 . Other homologues were also observed between $B$. subtilis and E. coli. However, the upstream information for those hits was not available, since they either had intergenic regions shorter than $150 \mathrm{nt}$ or they contained annotated regulatory elements which were excluded from the scanning procedure.

\section{Conclusion}

Riboswitches are comprised of a diversity of biological functionality, as well as having different conformational dynamics. In this work, we made an attempt to characterize the potential for an alternative fold (switching ability) ubiquitous in various regulatory elements, regardless of their annotation and structural complexities. Various tests showed that there is in fact a relationship between higher structural entropy and RNA switching 
ability. This relationship was shown to be modest but consistent across various tests. Furthermore, incorporating the structural entropy feature under the simplistic and symmetric RND folding model was shown to be informative in distinguishing riboswitches from their random shuffles. Unlike results from mutagenesis and antisense tests, the structural entropy feature of riboswitches was not on average higher than their corresponding random shuffles. Given both the diversity of conformational arrangement of gathered riboswitches and the simplicity of models used, there is potential for this feature in detecting RNA conformational switching and ultimately in de novo riboswitch discovery.

Classifiers based on structural entropy optimized through sequence and structural features were devised to distinguish between the putative riboswitch and the antisense control. They were also used as riboswitch identifiers in various prokaryotes. Potential shortcomings and considerations were also explored. Factors such as, length, organism the riboswitch is taken from, and the type of riboswitch should be considered in preparing a training set for future approaches. The lightweight RND folding model tended to have a very consistent and robust result in distinguishing extensive secondary structures from other intergenic regions on genome-wide scale, regardless of test parameters.

Structural entropy using stochastic context-free grammars provides a means to better understanding the conformational dynamics of the RNA, in general. Current modeling training techniques for SCFGs focus on higher accuracy to predict RNA secondary structure, and not necessarily higher accuracy of folding space or dynamics. Interestingly, the more simplistic RND folding model used in our approach had a higher performance than the more accurate BJK model, under serveral tests. The use of a more complex and accurate folding model may not necessarily result in a better exploration of the folding dynamics of non-coding RNAs. Fully understanding the behaviour of structural entropy may require extensive exploratory investigation, both from modeling and data collection perspectives.

\section{Methods}

\section{Data collection}

Sequences with concrete evidence of alternative structures were gathered from the literature (see Table 2). Prokaryotic sequences believed not to have structure were selected from E. coli and are listed in Table 9 as negative set. 30 genome locations corresponding to $\sigma-70$ transcription factor binding sites that are less than $80 \mathrm{nt}$ upstream of their corresponding start codon were randomly chosen from $E$. coli such that they are fairly evenly distributed across the genome. Data was manually gathered from the EcoCyc website (http://ecocyc.org/).

\section{Mutagenesis}

Sensitivity and specificity values of Table 5 were calculated from Table 4 . Sensitivity: the percentage of structural mutants (annotated by YES in Table 4) having lower values than the wild type (corresponding negative value in Table 4). Specificity: the percentage of non-structural mutants (annotated by NO in Table 4) having higher or equal values with respect to the wild type (corresponding positive or zero value in Table 4). Mathews Correlation Coefficient is shown in column MCC. Features Base-pairing entropy (BJKbp), 
Structural entropy under BJK and RND models, (BJK) and (RND), respectively, and two-cluster average silhouette index of energy landscape (Sil) were investigated. For the case of the Bradyrhizobium ROSE-P2, entropy values were compared with -0.74 rather than zero for wild type, since the length of the 135nt-long riboswitch was decreased by 1 and this decrease in length is expected to have linear effect on structural entropy values.

\section{Classification}

\section{Preparing the positive control set}

The criterion for building the positive control set was taking the minimum-length subsequence for the corresponding riboswitch with evidence for alternative structures. Comprehensive structure information was not available for certain sequences. We decided to include them to increase our data set size. The structures of most sequences were experimentally validated, although a few structures of the riboswitches were inferred in combination with structural homology approaches. Only the expression platform components for the Cobalamin riboswitches were used, since they contain alterations; a typical riboswitch has an aptamer and an expression platform component, where the aptamer binds to the ligand, triggering allosteric rearrangement of the conformation of the expression platform component of the riboswitch which in turn regulates the expression of the downstream gene. Cobalamin riboswitches are also significantly longer than other sequences, e.g. Salmonella enterica serovar Typhimurium's Cobalamin riboswitch was over $300 \mathrm{nt}$ long. Including such long sequences could have been problematic, for both sensitivity of structural entropy on sequence length and the fact that RNA structures longer than $200 \mathrm{nt}$ are usually predicted with low confidence under SCFG models as well as computational constraints. Also, certain sequences were excluded from the test. In the column Grouping of Table 2 we denote None for such sequences. Excluded sequences are as follows: Salmonella ATP regulatory element, located in the mgtM gene before the $m g t C B R$ operon, was excluded since it was the only RNA in our set that had complete overlap with codons [73]. Synechococcus sp. CC9902 Downstream-peptide motif was excluded, since evidence for alteration was not available. T. tengcongensis glmS ribozyme-riboswitch was excluded, since the glmS ribozyme does not undergo "large conformational changes concomitant with ligand binding" [74] and is the only RNA element in our gathered data that functions as a self-cleaving ribozyme upon binding to glucosamine-6-phosphate (GlcN6P) ligand [75]. Synechococcus elongatus glnA motif was excluded, since no evidence of alteration was available. Schistosoma Mansoni Hammerhead type I ribozyme was excluded, since its structure does not alter. The pseudoknotted marine metagenome Hammerhead type II ribozyme was also excluded, since there was no evidence of alteration of the secondary structure. Finally, Bacillus subtilis yxkD motif was excluded, since there was no concrete biological evidence for it being a functional riboswitch or ribo-regulator, although it is predicted partially to have an alternative structure [76].

\section{Choice of reverse-complement (Antisense) as a negative control}

As we know, riboswitches are under selective pressure to preserve their potential for alternative folds due to their biological role. The reverse-complement of the RNA or the antisense sequence was here assumed not to have potential for two alternative structures; 
they may have at most one structure, since they are complementary to the sense. A cisregulator has an alternative fold, typically through conformational rearrangements of the expression platform to be able to regulate the expression of the downstream genes in the same mRNA, while the antisense is not necessarily under such evolutionary pressure (See Background section for exceptions). Experimental work to back this assumption on the gathered riboswitches was not found. The following assumption was made: On average, a given set of validated riboswitch sequences are expected to contain more RNA switching features than their corresponding antisense sequences. Advantages and disadvantages of the choice of reverse complement (or antisense) are as follows: Advantages: In the reverse complement, Watson-Crick pairing positions are kept intact. This implies that the folding space of the antisense may contain secondary structural features similar to the MFE structure of the sense sequence, making it a near negative control. Other more established negative controls such as various sequence shuffling techniques do not guarantee this. In addition, high correlation of structural entropy values to nucleotide composition and length of the sequence [55] make antisense a convenient choice of negative control. Disadvantages: There may be possible co-association with other sequence features such as U-composition. Also, G-U pairs may differ between sense and antisense structures.

\section{Training and test sets}

The positive control set was divided into the training and test sets. While most gathered sequences were in the two organisms, B. subtilis and E. coli, they cover a variety of biological functions and structures. We were interested in a method that can identify potential for the RNA to have an alternative secondary structure from a thermodynamic perspective regardless of a specific function or a secondary structural conformation. Hence, the categorization was done such that each of the training and test sets would contain as many adiverse sequences and structures as possible. Furthermore, the training sequences contain those from E. coli while the test set contains those of B. subtilis. Riboswitches that did not exist in both gram-positive and gram-negatives were evenly distributed between the two sets. Division of data into training and test sets was a compromise between having as diverse riboswitches as possible and being able to assess significance of classification on riboswitches from phylogenetically distant organisms, namely the gram-positive $B$. subtilis and the gram-negative $E$. coli. In the column Grouping of Table 2, the categorization of each sequence is shown. There are a total of 29 sequences in the training set and 23 sequences, in the test set. The $30 \mathrm{E}$. coli UTRs were divided into sets of 17 and 13 for training and test sets, respectively. The categorization was selected such that for an extension of 100 nt UTRs upstream of their corresponding start codons, GC-composition, and the minimum free energy having similar distribution in both sets. A further internal control was the reverse-complement (or antisense) sequence of the riboswitch, adding additional sets of sequences of size 29 and 23 sequence to the training and test sets, respectively. Various classifications in this work always use antisense sequences of riboswitches of identical length for training and test sets, unless indicated otherwise. For the dinucleotide shuffling test, we originally generated 10 dinucleotide shuffles for each riboswitch using [60]. We then filtered the sequences and selected those having more similar MFE values to riboswitches based on the following criterion. For each of the training and test sets, we calculated average and standard deviation of the MFE values. We then eliminated those 
random shuffles having higher MFE values than average minus one standard deviation, for the training and test sets separately. The resulting statistics can be seen in Table 8. As we can see, the statistics for other features such as length and GC-composition were also altered.

\section{Classification criterion}

Classification probabilities of having an alternative fold (riboswitch), possibly only one fold (antisense), or no riboswitch structure (E. coli UTR) were assigned to each sequence based on multinomial logistic regression of sequences in the training. SPSS $16.0 \odot$ software was used to estimate the corresponding parameter vectors. Such parameter vectors were then used to calculate the probabilities of sequences in the test set to belong to each class using their features. All true and false positive rates presented (except the ROC curves) are based on maximum likelihood, where the probability with maximum value determines the class of the sequence in the test set. In this work and all presented tables, the term probability (or likelihood score) for being potential riboswitches refers to "trained output logistic model score" assigned to the sequence. Entropy calculations were done according to [55]. Two different lightweight context-free secondary structural models were used as folding distribution models. The first model, denoted here as BJK, was developed by Knudsen/Hein and originally used in the Pfold package [77,78]. The structurally unambiguous grammar was subsequently used in [79] under the name G6 to predict RNA secondary structure using different training sets for RNA secondary structures. Model parameters used here correspond to the benchmark-trained version of this grammar [79] and will be referred to as the BJK model. Average sensitivity and specificity values for the BJK model on the test set of riboswitches are 75.6 and 76.3, respectively. The second model, denoted here as RND was introduced in [55] under the name RND10. This model consists of a structurally ambiguous simple grammar with symmetric rules and probabilities set according to [55]. Also, an effort was done to convert non-stacking heavyweight grammars from $[13,59]$. Such grammars aim at mirroring the state-of-the-art thermodynamic folding models and are extremely sophisticated, requiring their specific software implementation. The translation of these models into our simple implementation eliminated many of its features. The resulting model did not yield the original accuracy to predict RNA secondary structure, nor was its entropy showing any significant performance in the classifier (data not shown). Minimum-free-energy calculation was done by Vienna@Software Package [58] using default parameters. Base-pairing entropy for the BJK model, denoted here as BJKbp, was calculated as defined in [54] (implementation by [55]). BJKbp calculation is according to ([61] Eq. 3), where natural logarithm is used for base-pairing entropy calculations: $-(1 / n) \times \sum_{i<j} P_{i, j} \times \log P_{i, j}$. Symbol $n$ denotes length of the RNA sequence and $P_{i, j}$ denotes the probability of pairing in positions $i$ and $j$, under the given SCFG model. The two-cluster average Silhouette index of energy landscape, denoted here as Sil, was calculated according to the pipeline used in [46] with the exception that we did not account for pseudoknots and only used MFE predictions of the Vienna@Software Package for prediction of structures. Also, in the case of random shuffles, we only used the fixed number of 500 structural samples to do the calculations. RNAShapes [49] was used to derive an array of most possible abstract shapes resembling RNA secondary structure. We used parameters -e $[-500,10]-p$ to calculate 
different conformation probabilities. we used the ratio of the two probabilities $p 1 / p 2$, where $p 1$ and $p 2$ are the highest and the second highest probabilities associated with the predictions. One sequence lead to only one possible conformation as an output, for which we arbitrary chose 100. A similar trend was followed using FFTbor [52] software package.

We also tried to explore $\mathrm{GC}$ composition information upstream of gathered sequences relative to that in the riboswitch which did not lead to significantly better results. Sequence-similarity method such, as BLAST $\odot$ and profile Hidden Markov Models were also examined as classifiers with the mentioned training and test sets. The pipeline was implemented according to [80]. These methods did not result in significant classification performance even after lowering the corresponding threshold to insignificant values.

\section{Genome-wide scan of the $B$. subtilis, $E$. coli, and S. elongatus intergenic regions}

Bacillus subtilis subsp. subtilis str. 168 (taxid:224308), Escherichia coli str. K-12 substr. MG1655 (GenBank@ID: U00096.2) and Synechococcus elongatus PCC 6301 were downloaded from the National Center for Biotechnology Information (NCBI)@ [81,82]. The newer version of $E$. coli str. K-12 genome (gb|U00096.3) was not used, since operon and $\sigma-70$ UTR locations were given in the old version. Corresponding locations of $E$. coli riboswitches in the old version were used, where necessary. The operon-location information file for B. subtilis was downloaded from [83]. Candidates consisted of sequence segments of lengths $100 \mathrm{nt}, 150 \mathrm{nt}$, and $157 \mathrm{nt}$. Each intergenic region was divided into segments of such length such that the most downstream segment in each intergenic region ends at the start codon. Only intergenic regions higher than $150 \mathrm{nt}$ were considered. True positives were defined based on sequence segments that had maximum overlap with the original coordinates of the riboswitches. The same process was applied to the $E$. coli genome. Operon locations for the $E$. coli genome were downloaded from the RegulonDB website [84]. Operon locations in E. coli also contained RNA elements in our data set. Hence, results for the genome-wide scan of $E$. coli did not contain any sequence within the operon locations and only contained non annotated regions. S. elongatus gene locations were downloaded from the MicrobesOnline Operon Predictions website $[85,86]$. $S$. elongatus intergenic regions were chosen in a similar fashion. Computational complexity of the genome-wide scan on the available cluster and using parallel computation took roughly several days for a window size of $200 \mathrm{nt}$ and overlap of $100 \mathrm{nt}$.

\section{Cross-organism riboswitch candidate selection}

We specifically looked for homologous genes that have riboswitch candidates in their upstream region in different organisms. First, from the top 100 hits in B. subtilis, we collected the genes that are associated with hits having maximum distance of $600 \mathrm{nt}$ to the start codon. We then used tblastx to find their homologues in E. coli with an e-value threshold of $1 \mathrm{e}-6$.

\section{Endnotes}

aPseudoknots, another RNA structural feature, are a kind of base-pairings that resemble structural knots and cannot be predicted via context-free grammars. Predictions of pseudoknots based on minimum free energy and context-sensitive grammars are possible, though computationally expensive [87]. 
${ }^{\text {b}}$ Structural entropy was observed to be positively correlated with the MFE for random computer-generated sequences and structures under the BJK model (the lower the MFE the lower its entropy). While structural entropy under the RND model was observed to be independent of MFE (data not shown). Hence, higher entropy (structural entropy) and lower MFE of riboswitches is an unexpected observation, at least from a random sequence perspective.

'Structure partially validated, partially predicted via Vienna Software where not available. ${ }^{\mathrm{d}}$ Ten nucleotides added to the structure with no structure.

epartially predicted.

f Structural Homology Inferred.

gredicted by pknotsRG@program [88].

${ }^{\mathrm{h}}$ Structural Homology Inferred.

i Predicted by Vienna.

jPartially predicted by Vienna.

\section{Appendix}

\section{Additional tables}

Performance of different classifiers was evaluated for both constant and variable choices of length for the riboswitches (Table 14). The performance of classifiers that substitute centroid free energy for minimum free energy is shown in Table 15.

Table 14 Classification performance for different choices of length

\begin{tabular}{|c|c|c|c|c|c|}
\hline \multicolumn{6}{|c|}{ Variable Length } \\
\hline Features & $T P \%$ & $F P_{1} \%$ & $F P_{2} \%$ & Sensitivity & Sig. \\
\hline L,MFE,GC,RND & 69.6 & 39.1 & 7.7 & 61 & MFE,GC \\
\hline L,MFE,GC,BJK & 87.0 & 34.8 & 0.0 & 71.2 & GC \\
\hline L,MFE,GC & 87.0 & 30.4 & 0.0 & 76.3 & L,MFE \\
\hline \multicolumn{6}{|l|}{100} \\
\hline Features & $T P \%$ & $F P_{1} \%$ & $F P_{2} \%$ & Sensitivity & Sig. \\
\hline MFE,GC,RND & 69.6 & 26.1 & 7.7 & 66.1 & - \\
\hline MFE,GC,BJK & 65.2 & 21.7 & 7.7 & 64.4 & - \\
\hline $\mathrm{MFE}, \mathrm{GC}$ & 56.5 & 21.7 & 15.4 & 64.4 & - \\
\hline \multicolumn{6}{|l|}{150} \\
\hline Features & $T P \%$ & $F P_{1} \%$ & $F P_{2} \%$ & Sensitivity & Sig. \\
\hline MFE,GC,RND & 69.6 & 26.1 & 23.1 & 57.6 & MFE,RND \\
\hline MFE,GC,BJK & 69.6 & 39.1 & 7.7 & 59.3 & MFE \\
\hline MFE,GC & 69.6 & 39.1 & 0.0 & 64.4 & - \\
\hline \multicolumn{6}{|l|}{200} \\
\hline Features & $T P \%$ & $F P_{1} \%$ & $F P_{2} \%$ & Sensitivity & Sig. \\
\hline MFE,GC,RND & 65.2 & 34.8 & 7.7 & 62.7 & MFE \\
\hline MFE,GC,BJK & 78.3 & 34.8 & 7.7 & 66.1 & MFE \\
\hline $\mathrm{MFE}, \mathrm{GC}$ & 82.6 & 39.1 & 0.0 & 76.3 & MFE \\
\hline
\end{tabular}

Sub-section Variable Length refers to results of actual sequence lengths for both training and test sets (equal number of varying sequence lengths of 100, 150, and 200 from E. coli UTR chosen as negative set). Sub-sections 100, 150, and 200 refers to results where all sequences in the training and test sets have a constant length. Column Features denotes features used from the training set. $T P \%$ denotes percentage of true positives. $F P_{1} \%$ and $F P_{2} \%$ represent the percentages of antisense sequences and $E$. coli UTRs that are misclassified as riboswitches, respectively. Sensitivity denotes overall percentage of correctly classified sequences. Sig. denotes significant (less than 0.05 in the training set) features of the multinomial classifier. 
Table 15 Classification performance using centroid free energy

\begin{tabular}{lccccc}
\hline Classifier & $\boldsymbol{T P} \%$ & $\boldsymbol{F P}_{\mathbf{1}} \%$ & $\boldsymbol{F P}_{\mathbf{2}} \%$ & Sensitivity & Sig. \\
\hline LCFEGCRND & 65.2 & 30.4 & 15.4 & 66.1 & CFE \\
LCFEGC & 78.3 & 56.5 & 15.4 & 61 & L,CFE \\
LCFEGCBJK & 82.6 & 65.2 & 15.4 & 59.3 & GC
\end{tabular}

Classifier Performance. Actual length of sequences used. Feature CFE denotes centroid free energy as calculated by CentroidFold@ [62]. Column Classifier denotes features used from the training set. TP\% denotes percentage of true positives. $F P_{1} \%$ and $F P_{2} \%$ represent the percentages of antisense sequences and $E$. coli UTRs that are misclassified as riboswitches, respectively. Sensitivity denotes overall percentage of correctly classified sequences. Sig. denotes significant (less than 0.05 in the training set) features of the multinomial classifier.

\section{Collected riboswitch sequences}

Riboswitch sequences and their corresponding secondary structures were collected from the literature. Riboswitches used in the training set are shown in Training set in Appendix. Riboswitches used in the test set are shown in Test set in Appendix. Sequences excluded from classification are shown in Excluded set.

\section{Training set}

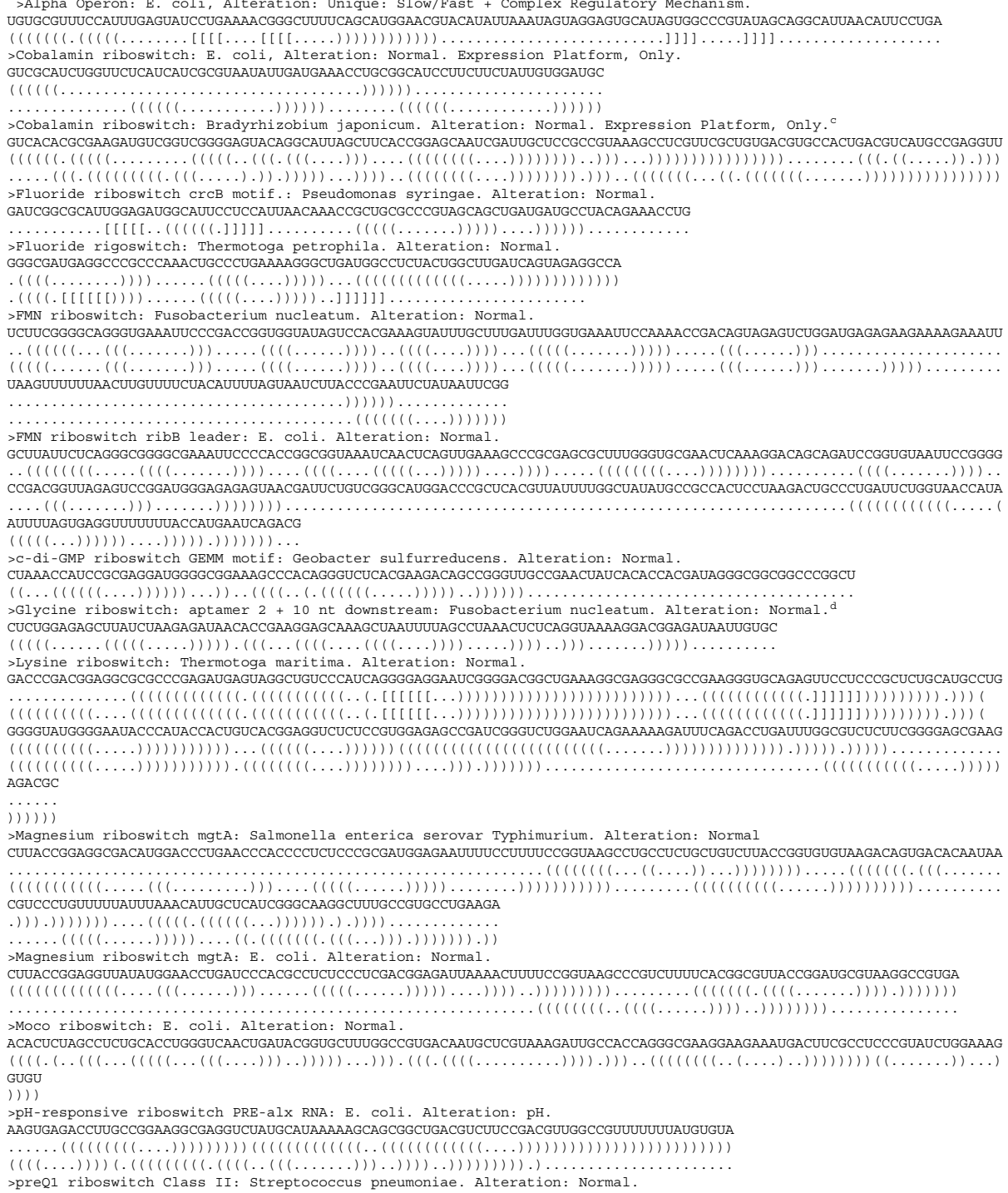


GUUGAAUGAAUCAACCCUUGGUGCUUAGCUUCUUUCACCAAGCAUAUUACACGCGGAUAACCGCCAAAGGAGAAAAGAUG

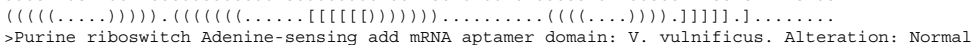

CGCUUCAUAUAAUCCUAAUGAUAUGGUUUGGGAGUUUCUACCAAGAGCCUUAAACUCUUGAUUAUGAAGUCUGUCGCUUUAUCCGAAAUUUUUAUUAAAGAGAAGACUCAUGAAU

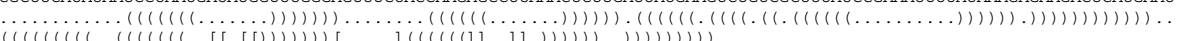

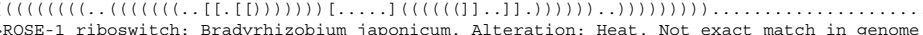

ROSE-1 Tiboswitch: Bradyrhizobium japonicum. Alteration: Heat. Not exact match in genome

GCGCGACAAGCGGUCCGGGCCCCUAGGGGCCCGGCGGAGACGGGCGCCGGAGGUGUCCGACGCCUGCUCGUACCCAUCUUGCUCCUUGGAGGAUUUGGCUAUGAGGA

ROSE-P2 riboswitch: Bradyrhizobium. Alteration: Heat. Not exact match with genome

GCCGCUUACGGGCGGUGCGCGGGCGCCAGGUUGGGCUCGGCCAAAGCAACCAGGGCGCCGGACAGGUGUCUUGCACCGAUCGUCUUGCGCUCCUUCGUAUCCAUCUUGCUCUCUG

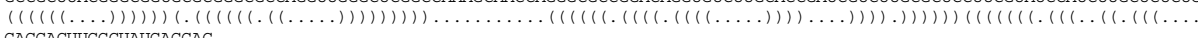
GAGGACUUGGCUAUGAGGAC

$(1) 11)(.11) .1111111)$.

ROSE-2 riboswitch: E. coli. Alteration: Heat.

UCUGGCUACCUGACCUGUCCAUUGUGGAAGGUCUUACAUUCUCGCUGAUUUCAGGAGCUAUUGAUUAUGCGUA

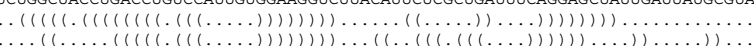

\SAH riboswitch upstream of ahCY: Ralstonia solanacearum. Alteration: Normal. Inferred structural homology.

AAGUUUGCGAUCCGCUAACCGGUCAAGCCGUGUCGCGGAAGGUUGAUGAACCCGCUGAACUCCGGCAGACCCGGAGAAAGGUGAGCGCCCCAUGACU

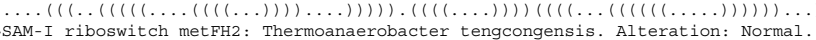

S

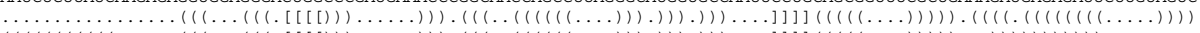

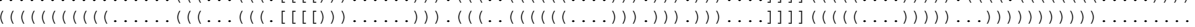
UCUUCUUUU

>SAM-II riboswitch metA: Agrobacterium tumefaciens. Alteration: Normal. Structural Homology Inferred. AGUGGUGAUUUGGCGGACCGGCUUGCAGCCACUUUAAAAGAAGUCGCUAAAGGGUCGAGGAAAAGGGCAAUUUCCUGGGACCGGCCGCGAUUUUCGCUGCCGG (2) $>$ SAM-III riboswitch SMK: Streptococcus gordonii. Alteration: Normal. AGUUAUAUAGUCCCGAUAAGAUGGUAGGAAACUUCUAUCAGUUCUUUGUAACUCUAUAAACAAUAUUUUUUUUAAAGGGGGGAC

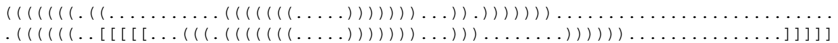
>SAM-IV riboswitch xylE gene: Streptomyces coelicolor. Alteration: Normal. Structural Homology Inferred. GGUCAUGAGUGACAGUCAUGAGGCCCCGGCCGACUGUCCGGCAACCCUCCGUCCGUGGCGGGGUGCCCCGGGUGAAGACCAGGUCGUGGACAGCAAGGUCCACGGCAAGCGCGGA

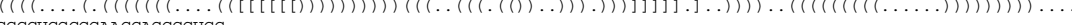
CCCCUCGCGGAACCAGGGGUCC

$>$ SAM-V riboswitch 62 metY: Candidatus Pelagibacter ubique. Alteration: Normal.

AGGCGCAUUUGAACUGUAUUGUACGCCUUGCAUAAAGCAAAAGUACUAAAAAA

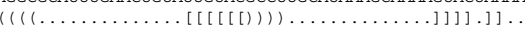

$>$ SAM-SAH riboswitch metK: Roseobacter sp. SK209-2-6. Alteration: Normal. Structural Homology Inferred.

CCUGUCACARCGGCUUCCUGGCGUGACG

$((((()((\ldots \ldots[[[[\ldots))))))).) \ldots \ldots \ldots .]]]]$.

$\rightarrow$ THF riboswitch folT: Eubacterium siraeum. Alteration: Normal.

ACAGAGUAGGUAAACGUGCGUAAAGUGCCUGAGGGACGGGGAGUUGUCCUCAGGACGAACACCGAAAGGUGGCGGUACGUUUACCGCAUCUCGCUGUUCCAUAUAAGAGAUAA

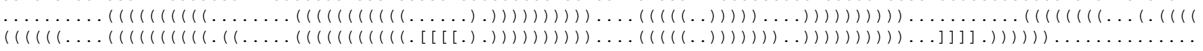
(I)

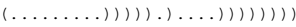

>TPP riboswitch thiM mRNA: E. coli. Alteration: Normal.

GACUCGGGGUGCCCUUCUGCGUGAAGGCUGAGAAAUACCCGUAUCACCUGAUCUGGAUAAUGCCAGCGUAGGGAAGUCACGGACCACCAGGUCAUUGCUUCUUCACGUUAUGGCA

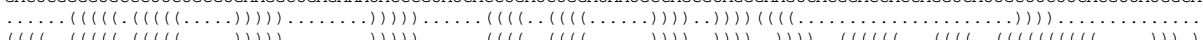
GGAGCAAACUAUGCAAGUCGACCUGCUGGGUUC

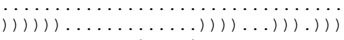

Tryptophan riboswitch trp Operon: E. coli. Alteration: Normal.

UGGUGGCGCACUUCCUGAAACGGGCAGUGUAUUCACCAUGCGUAAAGCAAUCAGAUACCCAGCCCGCCUAAUGAGCGGGCU

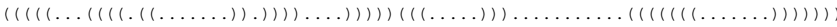

Test set

$>$ ATP riboswitch: B. subtilis. ydao Motif. Alteration: Normal.

AAUCGCUUAAUCUGAAAUCAGAGCGGGGGACCCAAUAGAACGGGUUUUUCCCGUAGGGGUGAAUCCUUUUUAGGUAGGGCUAACUCUCAUAUGCCCGAAUCCGUCAGCUAACCUC

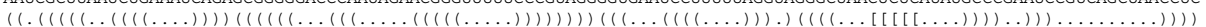
GUAAGCGUUCGUGAGAG

Cobalamin riboswitch: Salmonella. Alteration: Normal. Expression Platform, Only.

ACUUCGGUGGGAAGUGGGUUGCGAAGACGCGUACAGUCGAAAGACUGAACAUGCGCGUACCUGUAUACCCCUACCACCCUGACACAGGAUCAGGGU

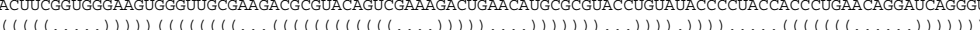

$\rightarrow$ Fluoride riboswitch: Bacillus cereus. Alteration: Normal.

UAGGCGAUGGAGUUCGCCAUAAACGCUGCUUAGCUAAUGACUCCUACCAGUAUCACUACUGGUAGGAGUCUAUU

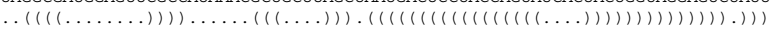

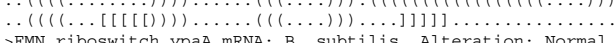

$>$ FMN riboswitch YpaA mRNA: B. subtilis. Alteration: Normal:

UAUCCUUCGGGGCAGGGUGGAAAUCCCGACCGGCGGUAGUAAAGCACAUUUGCUUUAGAGCCCGUGACCCGUGUGCAUAAGCACGCGGUGGAUUCAGUUUAAGGCUGAAGCCGACA

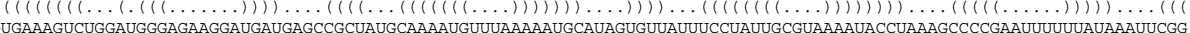

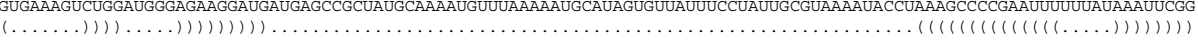
GGCUUU

$1) 1) 1$

$>$ C-di-GMP riboswitch GEMM motif: Candidatus Desulforudis. Alteration: Normal. ${ }^{\ddagger}$

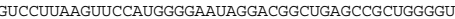

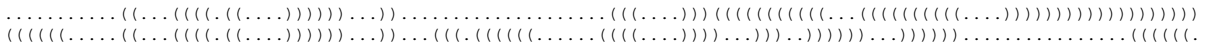
AUGGGGCGG

)$\ldots \ldots$

>Glycine riboswitch aptamer 2 + alteration of termination: Bacillus subtilis. Alteration: Normal.

.

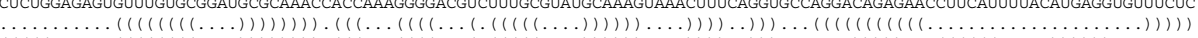

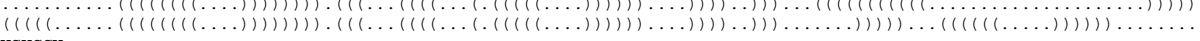
JGUCCU

>Lysine riboswitch: B. subtilis. Alteration: Normal. Not the genome version.

>LYsine riboswitch: B. subtilis. Alteration: Normal. Not the genome version. GGAUAGAGGUGCGAACUUCAAGAGUAUGCCUUUGGAGAAAGAUGGAUUCUGUGAAAAAGGCUGAAAGGGGAGCGUCGCCGAAGCAAAUAAAACCCCAUCGGUAUUAUUUGGCUGGC 
CGGGCAUUGAAUAAAUGUCAGGCUGUCAAGAAAUCAUUUUUCUUGGAGGGCUAUCC

$(((((((2 \ldots .)))))))))).(((((\ldots \ldots . . . . .1)))))))))))$.

$>$ Magnesium riboswitch M-box of Mgte gene: B. subtilis. Alteration: Normal.

CUUCGUUAGGUGAGGCUCCUGUAUGGAGAUACGCUGCUGCCCAAAAAUGUCCAAAGACGCCAAUGGGUCAACAGAAAUUCAUCGACAUAAGGUGAUUUUUUAAUGCAGCUGGAUGCU

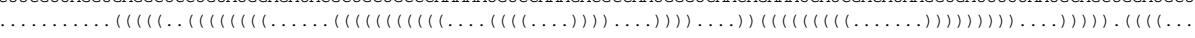

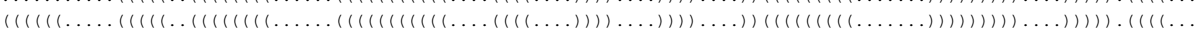
UGUCCUAUGGCAUACAGUGCUAAAGCUCUACGAUUGGAAGGCGCCCGCACGCUUUUUUUUGCCGUGCUUCUUUCACCUUCAAUCCCGAAGG

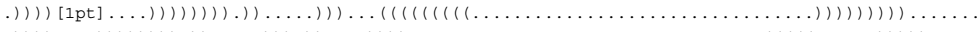

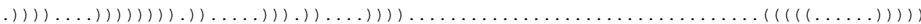

$>$ Tuco riboswitch. Geobacter metallireducens. Alteration: Normal.

UAGUUUUUUUCUCCGAUCCGUCAUACCUACCAGGCGCAGAGCCUCACGGUAUGCGGUCAACGGGUUCCGCUGGAAACGGCGGUGCCUCCCUUUUGGAAAGGAGAACUCUUUA

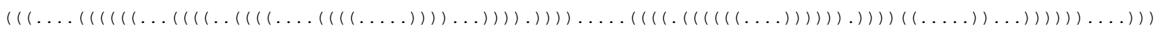

$>$ pH-responsive riboswitch: Serratia marcescens. Alteration: pH.

AAGUGAGACCUUGCCGGAAGGCGAGGUUUGCUUGCAGCGUUCAUAGAGCGGCUGGCGUCUUCCGACGUUGGCCGU

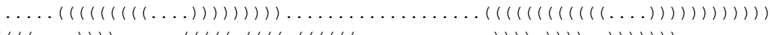

>preQ1 riboswitch Clo

AGAGGUUCUAGCUACACCCUCUAUAAAAAACUAAGGACGAGCUGUAUCCUUGGAUACGGC

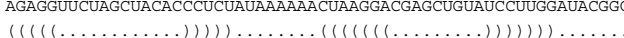

$((((([[[[\ldots . .)))).) \ldots \ldots]]]] \ldots \ldots((((((()((\ldots)))))))))$

>Purine riboswitch Adenine-sensing ydhL mRNA aptamer domain: B. subtilis. Alteration: Normal.

UUGUAUAACCUCAAUAAUAUGGUUUGAGGGUGUCUACCAGGAACCGUAAAAUCCUGAUUACAAAAUUUGUUUUUGACAUUUUUUGUUAAUCAGGAUUUUU

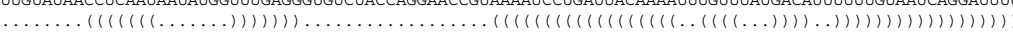

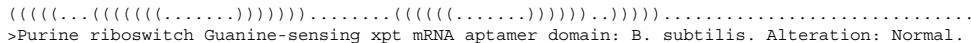

>Purine riboswitch Guanine-sensing xpt mRNA aptamer domain: B. subtilis. Alteration: Normal.

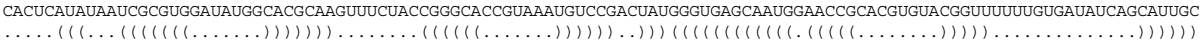

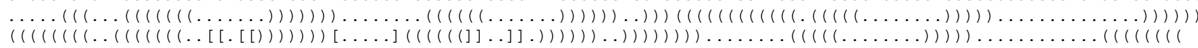

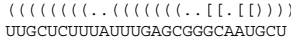

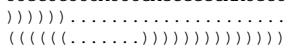

$((((() \ldots \ldots(\ldots)))()))()))))$
$>$ ROSE-N1 riboswitch: Rhizobium. Alteration: Heat.

>ROSE-N1 riboswitch: Rhizobium. Alteration: Heat.

GCCGAUGCCAAUUGGGUCGGCAUGGUCAGGGAGCGCCACGCUUCUUGGCGCUUCCUCGUAUCUAUGUUGCUCUACGGAGGAUGUAGCUAUGAGAAC
$(((((()(((\ldots)))))))) \ldots(((((((((1(\ldots)))))))))) \ldots \ldots(((((1 .((((((().(((\ldots))))))))))))).) \ldots$

$>$ ROSE-2387 riboswitch: Mesorhizobium loti. Alteration: Heat.

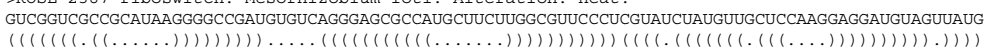

$>$ SAM-I riboswitch apo yitJ S-box: $B$ subtilis. Alteration. Normal.

>SAM-I riboswitch apo yitJ S-box: B. subtilis. Alteration: Normal.

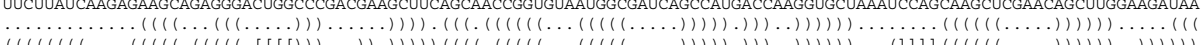

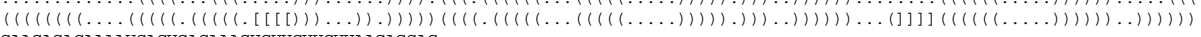
GAAGAGACAAAAUCACUGACAAAGUCUUCUUCUUAAGAGGAC

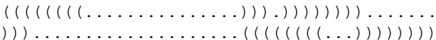

>SAM-III riboswitch metK SMK box: Enterococcus faecalis. Alteration: Normal.

SSAM-III riboswitch metK SMK box: Enterococcus faecalis. Alteration: Normal.

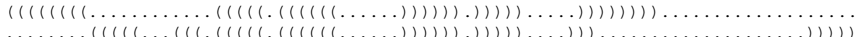

>SAM-IV riboswitch: Mycobacterium tuberculosis. Alteration: Normal.

CUAGGCUUCGAGUCGGUCAUGAGCGCCAGCGUCAAGCCCCGGCUUGCUGGCCGGCAACCCUCCAACCGCGGUGGGGUGCCCCGGGUGAUGACCAGGUUGAGUAGCCAUCGCCGGC

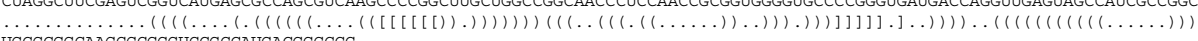
UGCGCGGCAAGCGCGGGUCCGCCAUGACGGGCCC

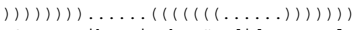

$>$ SAM-V riboswitch: Candidatus Pelagibacter ubique. Alteration: Normal. 9

AAUUAAGCCGGGCAGUUGAACCAUAUUGUGCGCCCUGCAUUUGCUUAAGCACUAAAAAGGAGAAA

$\ldots \ldots(().((().((\ldots \ldots \ldots \ldots \ldots)$
$>$ SAM-SAH riboswitch: Oceanibulbus indolifex. Alteration: Normal.

AGAGCAUCACAACGGCUUCCUGACGUGGUGCGUAAUUUUUUAUUGGAGCA

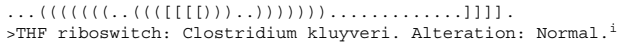

$>$ THF riboswitch: Clostridium kluyveri. Alteration: Normal. ${ }^{i}$

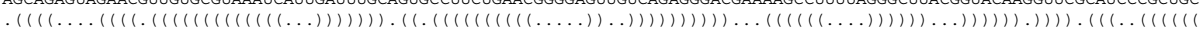
UGCAUAAGGAGAAGCGGUGUAAUGAUUGU

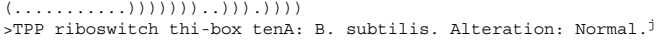

AACCACUAGGGGUGUCCUUCAUAAGGGCUGAGAUAAAAGUGUGACUUUUAGACCCUCAUAACUUGAACAGGUUCAGACCUGCGUAGGGAAGUGGAGCGGUAUUUGGUGUUAUUUUA

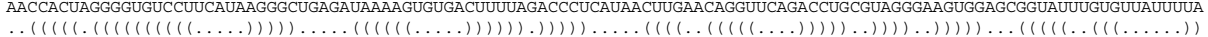
CUAUGCCAAUUCCAAACCACUUUUUCCUUGCGGGAAAGUGGUUUU

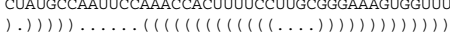

$>$ Tryptophan riboswitch trp Operon: B. subtilis. Alteration: Normal.

>Tryptophan riboswitch trp Operon: B. subtilis. Alteration: Normal.

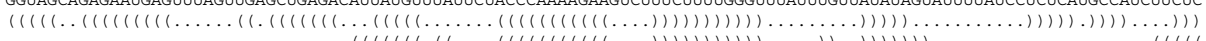
AUUCUCCUUGCCAUAAGGAGUGAGAG

$111) 11.11) 11) \ldots \ldots \ldots \ldots$

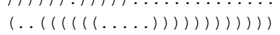

\section{Excluded set}

$>$ ATP Operon: Salmonella, Aleration: Unique: Temperature + Overlap with codons of mgtM. UGGCAAGUUAACGCACGCUAUUCCUGCGCUGCUUGCCGAACCGGUGGGCAGC

$((((((1(\ldots)(((1 \ldots \ldots \ldots)))) \ldots)))))))) \ldots \ldots \ldots \ldots$

>glms riboswitch: Thermoanaerobacter tengcongensis. Alteration: none.

AGCGCCUGGACUUAAAGCCTTAAGGCUUUAAGUUGACGAGGGCAGGGUUUAUCGAGACAUCGGCGGGUGCCCUGCGGUCUUCCUGCGACCGUUAGAGGACUGGUAAAACCACAGG

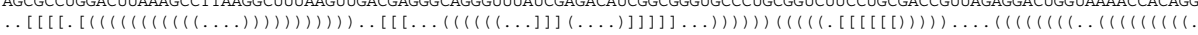
CGACUGUGGCAUAGAGCAGUCCGGGCAGGA

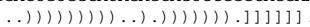

$>$ GlnA riboswitch: Synechococcus elongatus. Alteration: motif.

CGUUGGCCCAGUUUAUCUGGGUGGAAGUAAGGUCUUUGGCCUGAAGCAACGCGCCUCUCA

$(((((.(((((\ldots .)))).) \ldots[\ldots \ldots(((((\ldots))))) \ldots .)))))$.

>Downstream-peptide motif: Synechococcus sp. CC9902. Alteration: motif.

CGUUGAGCUUCCAAUCGAAGCUGCAGUCAGACCCAUGCCAAGCAACGGGGGCGUGGG

$(((()(((() \ldots \ldots))))) \ldots \ldots \ldots[[[[[[[[[\ldots)))))]]]]]]]$.

>Hammerhead ribozyme Type I: Schistosoma Mansoni. Alteration: none. Tertiary stability.

GCAGGUACAUCCAGCUGAUGAGUCCCAAAUAGGACGAAAUGCCGGCAUCCUGGAUUCCACUGC

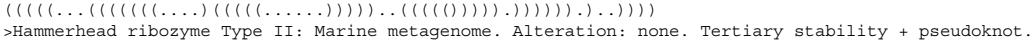

>Hammerhead ribozyme Type II: Marine metagenome. Alteration: none. Tertiar

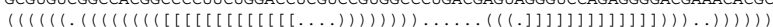




\section{Genome-wide scan results Bacillus subtilis}

In order to gain a better insight into the structural entropy of various riboswitches within their genomic context, in an exploratory attempt, we examined both the performance of classifiers and the high entropy (structural entropy) hypothesis on a genome level. In order to assess the performance of the classifier, we used regression values derived from the same training set to assign likelihood of being a riboswtich to various genomic locations of the B. subtilis genome. As a departure from classification method, we also searched for sequence segments that support our hypothesis; for that we collected sequence segments having both significantly high structural entropy and low MFE. Finally we explored the high structural entropy hypothesis in an inter-organism test, where we looked for homologous genes across the three distant organisms of B. subtilis, E. coli, and S. elongatus, that are all being associated with significantly high structural entropy values upstream of their coding region.

Eleven of the 23 riboswitches of the test set were also located in B. subtilis. The performance of the three tri-state classifiers on the eleven riboswitches and all other intergenic regions of the gram-positive bacterium, Bacillus subtilis are shown in Tables 16 and 17 in Appendix, for the actual variable lengths and constant lengths of the test set, respectively. In order to have a broader view of classification performance for the B. subtilis, we also replaced the antisense with sequences from intergenic regions, having same length and GC-composition as the sense sequences. The negative set consisted of eleven sequences and denoted here as $\mathrm{FP}_{3}$. Performance values are reported in Table 18 in Appendix.

Operon coordinates were taken from [83]. Performance of classifiers was higher for length $157 \mathrm{nt}$ rather than lengths $100 \mathrm{nt}, 150 \mathrm{nt}$, or $200 \mathrm{nt}$. This was true even though overlapping sliding windows were used for those lengths (sequence segment with highest overlap was selected as positive hit). In addition, we can see from Table 17 in Appendix that as window size increased, the number of intergenic regions classified as riboswitches $\left(T P_{2} \%\right)$ decreased. The classification performance of the LMFEGCRND model, however, was maximum at length $157 \mathrm{nt}$. (Length 157 was found using a rough optimization of various constant-length sequence selection and under the LMFEGCBJK model). We further examined the 157 nt length for two different sets of tests. In the first case, 157 nt-long segments were selected centered at the riboswitch (routine procedure) and in the second case, 157 nt extension of the 5' start of sequences were chosen. Classification performance is shown in Tables 16 and 19 in Appendix. Performance was very sensitive to positioning of the sequence segment of constant length. For the case of 5' selections, the LMFEGCBJK model outperformed other models having $T P \%=90.9$ while the centeredsegment test had a performance even lower than choosing random positioning. Hence,

Table 16 Classification performance in B. subtilis

\begin{tabular}{lcr}
\hline Classifier & $\boldsymbol{T P} \%$ & $\boldsymbol{F P _ { 1 } \%}$ \\
\hline LMFEGCBJK & 91.1 & 54.5 \\
LMFEGC & 81.2 & 36.4 \\
LMFEGCRND & 72.7 & 36.4 \\
\hline
\end{tabular}

Classifier Performance on the eleven B. subtilis riboswitches. Actual length of sequences used. Column Features denotes features used from the training set. TP\% denotes percentage of true positives. $F P_{1} \%$ represent the percentages of antisense sequences that are misclassified as riboswitches. 
Table 17 Classification performance for different choices of length in B. subtilis

\begin{tabular}{|c|c|c|}
\hline $100 \mathrm{nt}$ window & $T P \%$ & $F P_{2} \%$ \\
\hline LMFEGCRND & 63.6 & 29.8 \\
\hline LMFEGCBJK & 27.3 & 15.4 \\
\hline LMFEGC & 18.2 & 9.0 \\
\hline $150 \mathrm{nt}$ window & $T P \%$ & $F P_{2} \%$ \\
\hline LMFEGCRND & 72.7 & 20.5 \\
\hline LMFEGCBJK & 63.6 & 9.6 \\
\hline LMFEGC & 45.5 & 3.2 \\
\hline $157 \mathrm{nt}$ window & $T P \%$ & $F P_{2} \%$ \\
\hline LMFEGCRND & 81.8 & 19.5 \\
\hline LMFEGCBJK & 54.5 & 8.3 \\
\hline LMFEGC & 63.6 & 2.1 \\
\hline 200 nt window & $T P \%$ & $F P_{2} \%$ \\
\hline LMFEGCRND & 72.7 & 14.2 \\
\hline LMFEGCBJK & 45.5 & 6.7 \\
\hline LMFEGC & 18.2 & 1.3 \\
\hline
\end{tabular}

Classifier Performance on the eleven B. subtilis riboswitches. Constant length of $100 \mathrm{nt}, 150 \mathrm{nt}, 157 \mathrm{nt}$, and $200 \mathrm{nt}$ used for test. TP\% denotes percentage of true positives. $F P_{2} \%$ represent the overall percentage of sequences that are classified as riboswitches within the $B$. subtilis genome. $50 \mathrm{nt}, 75 \mathrm{nt}$, and $100 \mathrm{nt}$ overlaps were used for for sliding windows of lengths $100 \mathrm{nt}, 150 \mathrm{nt}$, and $200 \mathrm{nt}$, respectively. No overlaps were used for the $157 \mathrm{nt}$ window. True Positive sequences were according to maximum overlap with the location of the actual length of riboswitches.

Table 18 Classification performance in B. subtilis under 157 nt Length

\begin{tabular}{lccc}
\hline Classifier & $\boldsymbol{T P \%}$ & $\boldsymbol{F \boldsymbol { P } _ { \mathbf { 1 } } \%}$ & $\boldsymbol{F \boldsymbol { P } _ { \mathbf { 3 } } \%}$ \\
\hline L,MFE,GC,RND & 81.8 & 19.5 & 18.2 \\
L,MFE,GC & 63.6 & 2.1 & 0 \\
L,MFE,GC,BJK & 54.5 & 8.3 & 18.2 \\
\hline
\end{tabular}

Classifier performance on the eleven riboswitches in B. subtilis. Constant length of $157 \mathrm{nt}$ used. Sequence segments chosen according to the scanning procedure. Intergenic regions were divided into non-overlapping $157 \mathrm{nt}$ segments with most downstream segment ending at its corresponding downstream opron. Segments with maximum overlap with riboswitches were chosen as true positives. TP\% denotes percentages of correctly classified riboswitches. $F P_{1} \%$ denotes percentage of misclassified antisense. $F P_{3} \%$ denotes percentage of misclassified negative control segments. Average and standard deviation of the MFE values for the negative control segments are -30.7 and 8.2 , respectively.

Table 19 Classification performance in B. subtilis under constant length

\begin{tabular}{|c|c|c|c|c|}
\hline \multirow{2}{*}{$\begin{array}{l}\text { Segment } \\
\text { Classifier }\end{array}$} & \multicolumn{2}{|c|}{$5^{\prime}$} & \multicolumn{2}{|c|}{ Center } \\
\hline & $T P \%$ & $F P_{1} \%$ & $T P \%$ & $F P_{1} \%$ \\
\hline L,MFE,GC,BJK & 90.9 & 9.1 & 63.4 & 18.2 \\
\hline L,MFE,GC,RND & 54.5 & 0 & 63.4 & 36.4 \\
\hline L,MFE,GC & 72.7 & 9.1 & 63.4 & 18.2 \\
\hline
\end{tabular}

Classifier performance on the eleven riboswitches in B. subtilis. Constant length of $157 \mathrm{nt}$ from 5 ' of riboswitch downstream is used for riboswitches (first two columns). Constant length of $157 \mathrm{nt}$ centered at the center of riboswitches used (last two columns). $T P \%$ denotes percentages of correctly classified riboswitches. $F P_{1} \%$ denotes percentage of misclassified antisense. 
the LMFEGCBJK is more suitable for high performance where computational complexity is not an issue. For faster genome-wide tests where examining all sequence positions is not possible the LMFEGCRND seemed more appropriate $(T P=81.8 \%)$ and was based on selection of segments in a non-overlapping fashion, starting at the start codon for each operon. Selecting segments centered at riboswitches resulted in poor performance in $B$. subtilis.

The performance of classifiers on the eleven riboswitches were highly dependent on the length and positioning of sequence segments to be tested (see Tables 16, 17 and 19). Furthermore, various riboswitches had different sensitivities to such features (data not shown). We found that sequence segments of length $157 \mathrm{nt}$ resulted in higher performance compared to other lengths tested. Also, without knowledge of the exact location of the riboswitch, the LMFEGCRND model outperformed the LMFEGCBJK model, though the LMFEGCBJK model had a significantly higher performance if sequence segments were positioned at the right locations on the riboswitch. The likelihood for such desired positioning is very low; $1 / W L$ for each riboswitch, where $W L$ is the length of the non-overlapping sliding window.

The ranking of $B$. subtilis riboswitches using their actual length and constant length of 157 nt are shown in Tables 20 and 21, respectively. Classification probability of the LMFEGCRND model corresponding to the sequence segment overlapping with the TPP riboswitch (0.76) was higher than that of other riboswitches with ranking empirical pvalue 0.0122 . Results for the SAM-I riboswitch, however, were very poor. The actual length of the SAM-I riboswitch used in this study was also $157 \mathrm{nt}$.

Table 22 contains the top 50 best hits from each strand of the B. subtilis intergenic regions and their corresponding probability values. Sequence segments having classification probabilities higher than or equal to 0.8 fall in the top 50. Plot of structural entropy under the RND model and uracil composition of the sequence segments form the $B$. subtilis showed that structural entropy values were correlated with higher uracil composition (see Figure 5). This may have been partly due to the fact that uracil can bind with more nucleotides to form base-pairs under folding models. In order to suppress the effect of high uracil composition, we sorted the top hits having uracil compositions within the

Table 20 B. subtilis riboswitches ranking under actual-length test

\begin{tabular}{lr}
\hline Name & Probability \\
\hline Adenine & 0.82 \\
\hline FMN & 0.70 \\
\hline TPP & 0.68 \\
\hline Tryptophan & 0.67 \\
\hline Glycine & 0.63 \\
\hline Lysine & 0.63 \\
\hline Guanine & 0.60 \\
\hline ATP & 0.58 \\
\hline Magnesium & 0.54 \\
\hline SAM-I & 0.53 \\
\hline preQ1 & 0.451 \\
\hline
\end{tabular}

Ranking probabilities of the eleven $B$. subtilis riboswitches of $B$. subtilis under the LMFEGCBJK classifier. Actual sequence length used as test. Column Probability is the classification probability that the sequence is a riboswitch. 
Table 21 B. subtilis riboswitches ranking under constant-length test

\begin{tabular}{|c|c|c|c|c|}
\hline Name & Overlap & Rank & p-Value & Probability \\
\hline TPP & 82.9 & 347 & 0.0122 & 0.76 \\
\hline Guanine & 90.1 & 535 & 0.0189 & 0.735 \\
\hline ATP & 85.6 & 1159 & 0.0409 & 0.676 \\
\hline Lysine & 83.5 & 2278 & 0.0804 & 0.612 \\
\hline Adenine & 100 & 2459 & 0.0868 & 0.604 \\
\hline FMN & 51.7 & 3880 & 0.1369 & 0.547 \\
\hline preQ1 & 80 & 4051 & 0.1429 & 0.541 \\
\hline Magnesium & 62.3 & 4212 & 0.1486 & 0.536 \\
\hline Glycine & 91.7 & 5200 & 0.1835 & 0.508 \\
\hline Tryptophan & 100 & 6074 & 0.2143 & 0.484 \\
\hline SAM-I & 68.8 & 12330 & 0.4351 & 0.356 \\
\hline
\end{tabular}

Ranking probabilities of the eleven $B$. subtilis riboswitches within the $157 \mathrm{nt}$ non-overlaping window scan of the intergenic regions of $B$. subtilis under the LMFEGCRND classifier. Total of 28340 sequence segments belonging to intergenic regions longer than $150 \mathrm{nt}$ were analyzed. Operon coordinates: [83]. Overlap denotes the percentage of overlap of the sequence segment with the riboswitch. Column p-Value is the ranking divided by 28340 . Column Probabil ity is the classification probability that the sequence is a riboswitch.

range of known riboswitches in B. subtilis in Table 23. The location distribution of these hits can be seen in Figure 6. As we can see, the top hits do not seem to be associated with a specific genomic region as a whole. Sequence segments predicted to be riboswitches were not uniformly distributed across the genome. In order to investigate sequence location of segments with significant structural entropy values, regardless of their regression probabilities, we sorted segments having significantly low MFE (empirical p-value $<0.05$ ) while also having structural entropy values on the high 50 percentile. Hits with significant values that had GC and $\mathrm{U}$ compositions within the range of known riboswitches in B. subtilis are shown in Table 24. Interestingly, all of the hits also had significant structural entropy $\mathrm{p}$-values $(<0.05)$. P-values are calculated empirically and separately for each choice of window size in the genome-wide scan. Finally, significantly high structural entropy values of the 200 nt window scan that also have probability values higher than 0.8 , along with other significant hits, are available in Tables 25 and 26 regardless of their MFE or nucleotide compositions.

\section{Escherichia coli and Synechococcus elongatus}

Nine out of the 29 riboswitches in the training set are from the E. coli genome. As a test of the generality of the results on B. subtilis, we evaluated the performance of the three classifiers on various constant-length riboswitches, $100 \mathrm{nt}, 150 \mathrm{nt}, 157 \mathrm{nt}$, and 200 nt on E. coli. The performance of the LMFEGCRND classifier for the $100 \mathrm{nt}$-constant length was slightly higher than other tests (data not shown). Hence, the $100 \mathrm{nt}$ constantlength window scan of $50 \mathrm{nt}$ overlap was used to examine the intergenic regions of $E$. coli. The operon coordinates were taken from RegulonDB website [84]. Top 50 hits on each strands are available in Table 27. Top 50 hits having uracil compositions within the range of known riboswitches are organized in Table 28. The genomic distribution of the latter set is shown in Figure 6. Sequence segments having significant MFE and high structural entropy values are sorted in Tables 29 and 30 for significant and insignificant structural entropy values, respectively. 


\section{Table 22 Top classification hits in B. subtilis}

\begin{tabular}{|c|c|c|c|c|c|c|c|c|c|c|c|}
\hline$R$ & Start & End & Strand & Upstream operon & Upstream gene & Dist. to upstream & Uracil & Dist. to downstream & Downstream gene & Downstream operon & Probability \\
\hline 1 & 3717569 & 3717725 & reverse & BSU36100 & $y w r D$ & -1486 & 0.4076 & 550 & $\mathrm{cotH}$ & BSU36060 & 0.943 \\
\hline 2 & 3717412 & 3717568 & reverse & BSU36100 & $y w r D$ & -1643 & 0.4076 & 393 & $\cot \mathrm{H}$ & BSU36060 & 0.935 \\
\hline 3 & 4134175 & 4134331 & reverse & BSU40230 & yydA & -182 & 0.3439 & 79 & yydB & BSU40220 & 0.931 \\
\hline 4 & 3714883 & 3715039 & forward & BSU36030 & ywrk & -859 & 0.3949 & 2277 & $\cot G$ & BSU36070 & 0.922 \\
\hline 5 & 748990 & 749146 & forward & BSU06780 & yeec & -2912 & 0.414 & 707 & yeeG & BSU06820 & 0.919 \\
\hline 6 & 3666640 & 3666796 & reverse & BSU35680 & ggaB & -490 & 0.4968 & 1335 & mnaA & BSU35660 & 0.908 \\
\hline 7 & 3866327 & 3866483 & reverse & BSU37690 & $y w f G$ & -1881 & 0.3503 & 79 & eutD & BSU37660 & 0.903 \\
\hline 8 & 681153 & 681309 & forward & BSU06260 & ydjN & -201 & 0.3885 & 5731 & yeaB & BSU06320 & 0.899 \\
\hline 9 & 2987548 & 2987704 & reverse & BSU29200 & $\mathrm{acc} A$ & -104 & 0.4268 & 79 & pfkA & BSU29190 & 0.898 \\
\hline 10 & 1680274 & 1680430 & forward & BSU16080 & ylaH & 63 & 0.3822 & 79 & sucC & BSU16090 & 0.897 \\
\hline 11 & 2730227 & 2730383 & reverse & BSU26730 & yrdF & -254 & 0.4204 & 79 & $a z \mid B$ & BSU26720 & 0.896 \\
\hline 12 & 2316268 & 2316424 & reverse & BSU22040 & ypbQ & -99 & 0.363 & 236 & ypbR & BSU22030 & 0.896 \\
\hline 13 & 2219985 & 2220141 & forward & BSU20929 & yoyl & -6828 & 0.4204 & 2277 & yonP & BSU21030 & 0.896 \\
\hline 14 & 688027 & 688183 & forward & BSU06320 & yeaB & -114 & 0.3885 & 79 & yeac & BSU06330 & 0.893 \\
\hline 15 & 243578 & 243734 & forward & BSU02170 & $y b f B$ & -5370 & 0.363 & 236 & purT & BSU02230 & 0.89 \\
\hline 16 & 984466 & 984622 & reverse & BSU09120 & yhck & -1189 & 0.3885 & 79 & $\operatorname{cspB}$ & BSU09100 & 0.889 \\
\hline 17 & 2376780 & 2376936 & forward & BSU22510 & ypjC & -15199 & 0.4395 & 16564 & ypzl & BSU22869 & 0.888 \\
\hline 18 & 748205 & 748361 & forward & BSU06780 & yeeC & -2127 & 0.4395 & 1492 & yeeG & BSU06820 & 0.886 \\
\hline 19 & 3421066 & 3421222 & reverse & BSU33340 & sspJ & -320 & 0.3312 & 79 & lysP & BSU33330 & 0.885 \\
\hline 20 & 2093235 & 2093391 & forward & BSU19200 & desR & -852 & 0.4331 & 4789 & уоув & BSU19259 & 0.88 \\
\hline 21 & 3941212 & 3941368 & reverse & BSU38430 & gspA & -3269 & 0.4777 & 1649 & $y w b A$ & BSU38390 & 0.879 \\
\hline 22 & 1493630 & 1493786 & forward & BSU14230 & ykuV & -230 & 0.3503 & 79 & rok & BSU14240 & 0.879 \\
\hline 23 & 2531945 & 2532101 & forward & BSU24210 & yqiG & -14308 & 0.3439 & 9028 & yqhQ & BSU24490 & 0.879 \\
\hline 24 & 746478 & 746634 & forward & BSU06780 & yeeC & -400 & 0.5095 & 3219 & yeeG & BSU06820 & 0.878 \\
\hline 25 & 2096100 & 2096256 & reverse & BSU19230 & yocJ & -171 & 0.4268 & 393 & yocl & BSU19220 & 0.877 \\
\hline 26 & 300673 & 300829 & forward & BSU02770 & ycck & -1196 & 0.3822 & 79 & $y c d B$ & BSU02790 & 0.875 \\
\hline 27 & 3373963 & 3374119 & reverse & BSU32890 & yusQ & -2575 & 0.4076 & 393 & fadM & BSU32850 & 0.874 \\
\hline
\end{tabular}


Table 22 Top classification hits in B. subtilis (Continued)

\begin{tabular}{|c|c|c|c|c|c|c|c|c|c|c|c|}
\hline 28 & 3686143 & 3686299 & forward & BSU35770 & $\operatorname{tag} C$ & -1298 & 0.4586 & 2591 & gerBA & BSU35800 & 0.87 \\
\hline 29 & 1335487 & 1335643 & reverse & BSU12820 & spollsB & -12876 & 0.5032 & 13895 & xre & BSU12510 & 0.868 \\
\hline 30 & 4139318 & 4139474 & forward & BSU40240 & yycs & -3475 & 0.3567 & 864 & $\operatorname{rapG}$ & BSU40300 & 0.865 \\
\hline 31 & 1268672 & 1268828 & forward & BSU11970 & yjcS & -1 & 0.4458 & 79 & yjdA & BSU11980 & 0.865 \\
\hline 32 & 3685829 & 3685985 & forward & BSU35770 & $\operatorname{tag} C$ & -984 & 0.414 & 2905 & gerBA & BSU35800 & 0.865 \\
\hline 33 & 3681213 & 3681369 & forward & BSU35670 & gtaB & -14627 & 0.363 & 79 & $\operatorname{tag} \mathrm{A}$ & BSU35750 & 0.864 \\
\hline 34 & 1122705 & 1122861 & forward & BSU10490 & sipV & 55 & 0.4013 & 79 & yhjG & BSU10500 & 0.86 \\
\hline 35 & 3671690 & 3671846 & reverse & BSU35700 & $\operatorname{tag} \mathrm{H}$ & -1795 & 0.3694 & 393 & ggaA & BSU35690 & 0.859 \\
\hline 36 & 2160701 & 2160857 & forward & BSU20000 & yosU & -1938 & 0.4395 & 9028 & yosA & BSU20190 & 0.859 \\
\hline 37 & 1097850 & 1098006 & reverse & BSU10230 & yhfH & -191 & 0.465 & 79 & gltT & BSU10220 & 0.858 \\
\hline 38 & 1467020 & 1467176 & forward & BSU13960 & ykwC & -342 & 0.414 & 707 & $\mathrm{pbpH}$ & BSU13980 & 0.857 \\
\hline 39 & 191850 & 192006 & forward & BSU01590 & ybas & -12186 & 0.3057 & 2277 & $\operatorname{trnSL-Glu2~}$ & BSU_tRNA_75 & 0.856 \\
\hline 40 & 20723 & 20879 & forward & BSU00120 & yaaE & -86 & 0.3185 & 79 & serS & BSU00130 & 0.856 \\
\hline 41 & 2691445 & 2691601 & reverse & BSU26240 & yqaO & -1121 & 0.3439 & 79 & yqaQ & BSU26220 & 0.852 \\
\hline 42 & 3158851 & 3159007 & reverse & BSU30890 & $\mathrm{yt} \times \mathrm{O}$ & -328 & 0.363 & 3376 & ytdA & BSU30850 & 0.852 \\
\hline 43 & 1958206 & 1958362 & forward & BSU18190 & yngC & -9863 & 0.3949 & 44353 & iseA & BSU18380 & 0.852 \\
\hline 44 & 557716 & 557872 & forward & BSU05100 & yddT & -188 & 0.3503 & 79 & $y d z N$ & BSU05109 & 0.851 \\
\hline 45 & 3907629 & 3907785 & reverse & BSU38100 & ywcH & -2594 & 0.3567 & 393 & $y w c l$ & BSU38080 & 0.851 \\
\hline 46 & 1926523 & 1926679 & forward & BSU17950 & yneJ & -1482 & 0.3949 & 79 & citB & BSU18000 & 0.851 \\
\hline 47 & 1017271 & 1017427 & forward & BSU09400 & spoVR & -139 & 0.363 & 1649 & lytE & BSU09420 & 0.85 \\
\hline 48 & 1493595 & 1493751 & reverse & BSU14250 & yknT & -729 & 0.4522 & 1649 & ykuT & BSU14210 & 0.85 \\
\hline 49 & 2477743 & 2477899 & forward & BSU23830 & yqjL & 66 & 0.4076 & 1335 & zwf & BSU23850 & 0.849 \\
\hline 50 & 2769617 & 2769773 & reverse & BSU27160 & сурB & -4194 & 0.3185 & 1021 & yrhP & BSU27100 & 0.849 \\
\hline 51 & 2739991 & 2740147 & reverse & BSU26830 & yrpE & -1287 & 0.3694 & 3533 & aadk & BSU26790 & 0.849 \\
\hline 52 & 644384 & 644540 & forward & BSU05940 & $g c p$ & -7 & 0.3694 & 2120 & moac & BSU05960 & 0.848 \\
\hline 53 & 4039599 & 4039755 & forward & BSU39100 & yxiO & -23552 & 0.4713 & 1806 & hutP & BSU39340 & 0.847 \\
\hline 54 & 2203622 & 2203778 & forward & BSU20580 & yogM & -7279 & 0.363 & 79 & yops & BSU20780 & 0.847 \\
\hline 55 & 3014345 & 3014501 & reverse & BSU29460 & moaB & -90 & 0.3694 & 79 & $\operatorname{argG}$ & BSU29450 & 0.847 \\
\hline
\end{tabular}


Table 22 Top classification hits in B. subtilis (Continued)

\begin{tabular}{|c|c|c|c|c|c|c|c|c|c|c|c|}
\hline 56 & 749147 & 749303 & forward & BSU06780 & yeeC & -3069 & 0.363 & 550 & yeeG & BSU06820 & 0.846 \\
\hline 57 & 665425 & 665581 & forward & BSU06130 & ydjC & -677 & 0.3439 & 1963 & gutB & BSU06150 & 0.846 \\
\hline 58 & 2106272 & 2106428 & reverse & BSU19360 & odhB & -1154 & 0.3949 & 79 & yocR & BSU19340 & 0.846 \\
\hline 59 & 226409 & 226565 & forward & BSU02050 & ybdO & -82 & 0.3885 & 79 & $y b x G$ & BSU02060 & 0.844 \\
\hline 60 & 2106333 & 2106489 & forward & BSU19330 & sodF & -1353 & 0.3885 & 79 & yocs & BSU19350 & 0.844 \\
\hline 61 & 308175 & 308331 & forward & BSU02840 & $y c d G$ & 48 & 0.3503 & 79 & $\operatorname{adcA}$ & BSU02850 & 0.843 \\
\hline 62 & 2678925 & 2679081 & forward & BSU26050 & yqdB & -427 & 0.363 & 12639 & yqaP & BSU26230 & 0.843 \\
\hline 63 & 3875571 & 3875727 & reverse & BSU37760 & rocC & -130 & 0.3121 & 79 & ywfA & BSU37750 & 0.843 \\
\hline 64 & 2433680 & 2433836 & reverse & BSU23340 & ypuB & -384 & 0.3885 & 236 & ypzl & BSU23328 & 0.843 \\
\hline 65 & 2879134 & 2879290 & reverse & BSU28190 & engB & -669 & 0.4013 & 79 & hemA & BSU28170 & 0.842 \\
\hline 66 & 1533806 & 1533962 & forward & BSU14610 & pdhD & -445 & 0.3503 & 236 & ykzW & BSU14629 & 0.841 \\
\hline 67 & 368137 & 368293 & forward & BSU03360 & yciC & -802 & 0.3312 & 1021 & yckC & BSU03390 & 0.841 \\
\hline 68 & 447000 & 447156 & forward & BSU03930 & gdh & -792 & 0.3121 & 2120 & $y c n L$ & BSU03970 & 0.84 \\
\hline 69 & 3726630 & 3726786 & forward & BSU36160 & ywqM & -2216 & 0.4331 & 7144 & $y w q B$ & BSU36270 & 0.84 \\
\hline 70 & 543132 & 543288 & reverse & BSU05000 & yddK & -2955 & 0.4904 & 11697 & immR & BSU04820 & 0.84 \\
\hline 71 & 3268320 & 3268476 & forward & BSU31810 & yuzE & -4017 & 0.4522 & 8557 & yukF & BSU31920 & 0.84 \\
\hline 72 & 2065804 & 2065960 & forward & BSU18960 & yozM & -348 & 0.3758 & 8557 & yobN & BSU19020 & 0.839 \\
\hline 73 & 45296 & 45452 & reverse & BSU01550 & gerD & -113140 & 0.3822 & 236 & $a b r B$ & BSU00370 & 0.837 \\
\hline 74 & 2048779 & 2048935 & reverse & BSU18810 & yobA & -1092 & 0.363 & 550 & yoaZ & BSU18790 & 0.836 \\
\hline 75 & 3153718 & 3153874 & reverse & BSU30850 & ytdA & -938 & 0.3439 & 79 & menF & BSU30830 & 0.836 \\
\hline 76 & 3388260 & 3388416 & reverse & BSU33040 & fumc & -685 & 0.3312 & 393 & yuzO & BSU33029 & 0.834 \\
\hline 77 & 205252 & 205408 & forward & BSU01820 & $\mathrm{adaB}$ & -283 & 0.3248 & 79 & ndhF & BSU01830 & 0.834 \\
\hline 78 & 469269 & 469425 & forward & BSU04160 & $m t / R$ & 24 & 0.3121 & 79 & ydaB & BSU04170 & 0.834 \\
\hline 79 & 1868460 & 1868616 & forward & BSU17360 & $y m z A$ & -7 & 0.3885 & 79 & $\mathrm{nrdl}$ & BSU17370 & 0.834 \\
\hline 80 & 3746069 & 3746225 & forward & BSU36380 & rapD & -577 & 0.3822 & 2905 & $y w o H$ & BSU36440 & 0.833 \\
\hline 81 & 3467327 & 3467483 & reverse & BSU33800 & opuCD & -140 & 0.3376 & 79 & sdpR & BSU33790 & 0.832 \\
\hline 82 & 1264932 & 1265088 & reverse & BSU11990 & yjdB & -4722 & 0.5223 & 79 & yjcM & BSU11910 & 0.832 \\
\hline 83 & 1903262 & 1903418 & reverse & BSU17690 & yncm & -170 & 0.3822 & 1963 & $\cot U$ & BSU17670 & 0.831 \\
\hline
\end{tabular}


Table 22 Top classification hits in B. subtilis (Continued)

\begin{tabular}{|c|c|c|c|c|c|c|c|c|c|c|c|}
\hline 84 & 4204441 & 4204597 & reverse & BSU40960 & parB & -1036 & 0.414 & 79 & yyaD & BSU40940 & 0.831 \\
\hline 85 & 1017114 & 1017270 & forward & BSU09400 & spoVR & 18 & 0.3376 & 1806 & lytE & BSU09420 & 0.831 \\
\hline 86 & 2709577 & 2709733 & reverse & BSU26490 & yrkJ & -346 & 0.3503 & 236 & yrkK & BSU26480 & 0.829 \\
\hline 87 & 955738 & 955894 & forward & BSU08780 & ygaJ & -74 & 0.3822 & 79 & thiC & BSU08790 & 0.828 \\
\hline 88 & 554386 & 554542 & reverse & BSU05130 & $y d e B$ & -5686 & 0.4395 & 1963 & $\operatorname{IrpB}$ & BSU05060 & 0.828 \\
\hline 89 & 3988764 & 3988920 & reverse & BSU38860 & gale & -1105 & 0.293 & 79 & $y x k D$ & BSU38840 & 0.825 \\
\hline 90 & 2186812 & 2186968 & reverse & BSU20420 & yorD & -94 & 0.2611 & 79 & yorE & BSU20410 & 0.825 \\
\hline 91 & 2926840 & 2926996 & reverse & BSU28630 & pheT & -89 & 0.3185 & 1021 & yshA & BSU28610 & 0.823 \\
\hline 92 & 2054401 & 2054557 & reverse & BSU18840 & xynA & -119 & 0.3822 & 550 & pps & BSU18830 & 0.822 \\
\hline 93 & 610963 & 611119 & reverse & BSU05660 & ydgl & -1149 & 0.3121 & 2277 & $\operatorname{din} B$ & BSU05630 & 0.822 \\
\hline 94 & 3457144 & 3457300 & reverse & BSU33700 & opuBD & -2583 & 0.3185 & 707 & $y v z C$ & BSU33650 & 0.821 \\
\hline 95 & 736435 & 736591 & reverse & BSU06740 & yefB & -2481 & 0.3376 & 3690 & yerO & BSU06700 & 0.82 \\
\hline 96 & 2061478 & 2061634 & reverse & BSU18930 & yobH & -1953 & 0.3439 & 864 & yozJ & BSU18900 & 0.82 \\
\hline 97 & 2262616 & 2262772 & reverse & BSU21440 & $b d b B$ & -2530 & 0.3885 & 15151 & youB & BSU21329 & 0.819 \\
\hline 98 & 4118717 & 4118873 & reverse & BSU40110 & bglA & -2370 & 0.3758 & 5574 & glxK & BSU40040 & 0.818 \\
\hline 99 & 4204755 & 4204911 & reverse & BSU40960 & parB & -722 & 0.3949 & 393 & yyaD & BSU40940 & 0.818 \\
\hline 100 & 3648264 & 3648420 & reverse & BSU35530 & $\operatorname{tagO}$ & -311 & 0.363 & 1806 & $\operatorname{degs}$ & BSU35500 & 0.818 \\
\hline
\end{tabular}

Top 50 hits of the forward and reverse strands of the $B$. subtilis intergenic regions using no-overlap $157 \mathrm{nt}$ window and under the LMFEGCRND model. The ranking of each hit is denoted in column R. Distance from upstream and downstream operons are the distance from the center of the hit to the stop and start codons of upstream and downstream operons, respectively. Probability denotes the multinomial regression likelihood of being a riboswitch under the LMFEGCRND model.

Interesting hits are shown in bold. 


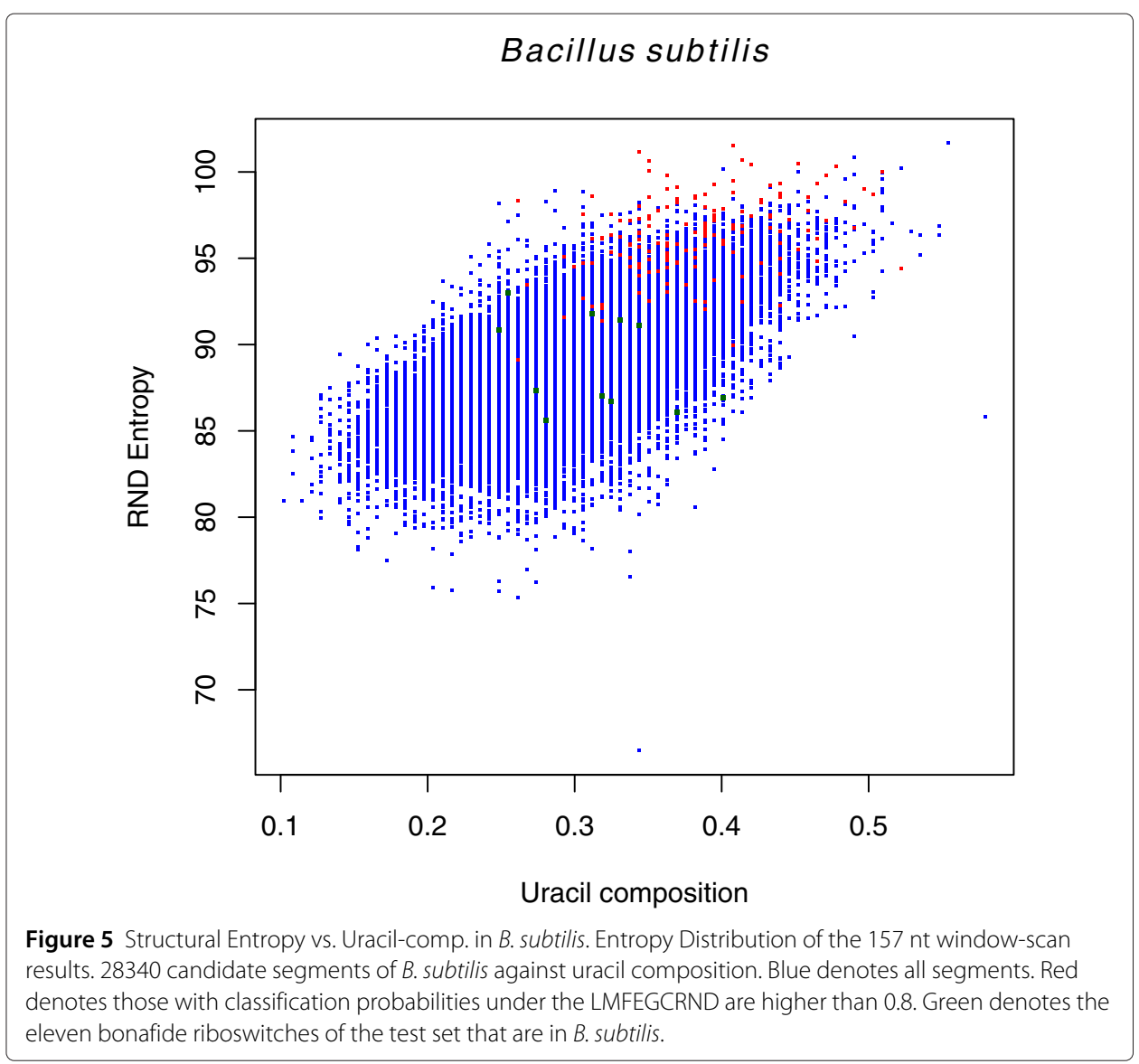

Synechococcus elongatus is another gram-negative bacterium which belongs to cyanobacteria. This organism is able to survive in freshwater environments with low nutrients. Intergenic regions of the sequenced strain Synechococcus elongatus PCC 6301 were scanned for riboswitch identification using the LMFEGCRND classifier with sliding window of $150 \mathrm{nt}$ and $75 \mathrm{nt}$ overlaps. Scanning procedure was similar to other organisms. The resulting top 100 hits (top 50 hits of the forward and reverse strands) are sorted in Table 31 according to their classification probabilities of being riboswitches under the LMFEGCRND classifier.

\section{Genome-wide scan results: tables}

Classification performance on the B. subtilis riboswitches is shown in Table 16. Classification performance on $B$. subtilis riboswitches using different choices of length is shown in Table 17. Performance values for the choice of $157 \mathrm{nt}$ as the riboswitch length are shown in Tables 18 and 19 for maximum overlap and relative positioning, respectively. Ranking of probabilities associated with different riboswitches are shown in Tables 20 and 21. Genome-wide scan results for B. subtilis are shown in Tables 22, 23, 24, 25 and 26 (please refer to Table captions for further explanation). Results for E. coli are shown in Tables 27, 28, 29 and 30. Results for S. elongatus are shown in Table 31. 
Table 23 Top classification hits in B. subtilis uracil-comp. constrained

\begin{tabular}{|c|c|c|c|c|c|c|c|c|c|c|c|}
\hline $\mathbf{R}$ & Start & End & Strand & Upstream operon & Upstream gene & Dist. to upstream & Uracil & Dist. to downstream & Downstream gene & Downstream operon & Probability \\
\hline 1 & 4134175 & 4134331 & reverse & BSU40230 & $y y d A$ & -182 & 0.3439 & 79 & yydB & BSU40220 & 0.931 \\
\hline 2 & 3714883 & 3715039 & forward & BSU36030 & ywrk & -859 & 0.3949 & 2277 & $\cot G$ & BSU36070 & 0.922 \\
\hline 3 & 3866327 & 3866483 & reverse & BSU37690 & ywfG & -1881 & 0.3503 & 79 & eutD & BSU37660 & 0.903 \\
\hline 4 & 681153 & 681309 & forward & BSU06260 & ydjN & -201 & 0.3885 & 5731 & yeaB & BSU06320 & 0.899 \\
\hline 5 & 1680274 & 1680430 & forward & BSU16080 & $\mathrm{ylqH}$ & 63 & 0.3822 & 79 & succ & BSU16090 & 0.897 \\
\hline 6 & 2316268 & 2316424 & reverse & BSU22040 & ypbQ & -99 & 0.363 & 236 & ypbR & BSU22030 & 0.896 \\
\hline 7 & 688027 & 688183 & forward & BSU06320 & yeaв & -114 & 0.3885 & 79 & yeaC & BSU06330 & 0.893 \\
\hline 8 & 243578 & 243734 & forward & BSU02170 & ybfB & -5370 & 0.363 & 236 & purT & BSU02230 & 0.89 \\
\hline 9 & 984466 & 984622 & reverse & BSU09120 & yhck & -1189 & 0.3885 & 79 & $\operatorname{cspB}$ & BSU09100 & 0.889 \\
\hline 10 & 3421066 & 3421222 & reverse & BSU33340 & sspJ & -320 & 0.3312 & 79 & lysP & BSU33330 & 0.885 \\
\hline 11 & 1493630 & 1493786 & forward & BSU14230 & ykuV & -230 & 0.3503 & 79 & rok & BSU14240 & 0.879 \\
\hline 12 & 2531945 & 2532101 & forward & BSU24210 & yqiG & -14308 & 0.3439 & 9028 & yqhQ & BSU24490 & 0.879 \\
\hline 13 & 300673 & 300829 & forward & BSU02770 & ycck & -1196 & 0.3822 & 79 & $y c d B$ & BSU02790 & 0.875 \\
\hline 14 & 4139318 & 4139474 & forward & BSU40240 & yycs & -3475 & 0.3567 & 864 & rapG & BSU40300 & 0.865 \\
\hline 15 & 3681213 & 3681369 & forward & BSU35670 & gtaB & -14627 & 0.363 & 79 & $\operatorname{tag} \mathrm{A}$ & BSU35750 & 0.864 \\
\hline 16 & 3671690 & 3671846 & reverse & BSU35700 & $\operatorname{tagH}$ & -1795 & 0.3694 & 393 & ggaA & BSU35690 & 0.859 \\
\hline 17 & 191850 & 192006 & forward & BSU01590 & ybas & -12186 & 0.3057 & 2277 & trnSL-Glu2 & BSU_tRNA_75 & 0.856 \\
\hline 18 & 20723 & 20879 & forward & BSU00120 & yaaE & -86 & 0.3185 & 79 & serS & BSU00130 & 0.856 \\
\hline 19 & 2691445 & 2691601 & reverse & BSU26240 & yqaO & -1121 & 0.3439 & 79 & yqaQ & BSU26220 & 0.852 \\
\hline 20 & 3158851 & 3159007 & reverse & BSU30890 & $y t x O$ & -328 & 0.363 & 3376 & $y t d A$ & BSU30850 & 0.852 \\
\hline 21 & 1958206 & 1958362 & forward & BSU18190 & yngC & -9863 & 0.3949 & 44353 & iseA & BSU18380 & 0.852 \\
\hline 22 & 557716 & 557872 & forward & BSU05100 & yddT & -188 & 0.3503 & 79 & $y d z N$ & BSU05109 & 0.851 \\
\hline 23 & 3907629 & 3907785 & reverse & BSU38100 & $y w c H$ & -2594 & 0.3567 & 393 & $y w c l$ & BSU38080 & 0.851 \\
\hline 24 & 1926523 & 1926679 & forward & BSU17950 & yneJ & -1482 & 0.3949 & 79 & citB & BSU18000 & 0.851 \\
\hline 25 & 1017271 & 1017427 & forward & BSU09400 & spoVR & -139 & 0.363 & 1649 & lytE & BSU09420 & 0.85 \\
\hline 26 & 2769617 & 2769773 & reverse & BSU27160 & сурB & -4194 & 0.3185 & 1021 & yrhP & BSU27100 & 0.849 \\
\hline 27 & 2739991 & 2740147 & reverse & BSU26830 & yrpE & -1287 & 0.3694 & 3533 & aadk & BSU26790 & 0.849 \\
\hline
\end{tabular}


Table 23 Top classification hits in B. subtilis uracil-comp. constrained (Continued)

\begin{tabular}{|c|c|c|c|c|c|c|c|c|c|c|c|}
\hline 28 & 644384 & 644540 & forward & BSU05940 & gcp & -7 & 0.3694 & 2120 & moaC & BSU05960 & 0.848 \\
\hline 29 & 2203622 & 2203778 & forward & BSU20580 & yogM & -7279 & 0.363 & 79 & yops & BSU20780 & 0.847 \\
\hline 30 & 3014345 & 3014501 & reverse & BSU29460 & moaB & -90 & 0.3694 & 79 & $\operatorname{argG}$ & BSU29450 & 0.847 \\
\hline 31 & 749147 & 749303 & forward & BSU06780 & yeec & -3069 & 0.363 & 550 & yeeG & BSU06820 & 0.846 \\
\hline 32 & 665425 & 665581 & forward & BSU06130 & ydjC & -677 & 0.3439 & 1963 & gutB & BSU06150 & 0.846 \\
\hline 33 & 2106272 & 2106428 & reverse & BSU19360 & odhB & -1154 & 0.3949 & 79 & yocR & BSU19340 & 0.846 \\
\hline 34 & 226409 & 226565 & forward & BSU02050 & ybdO & -82 & 0.3885 & 79 & $y b x G$ & BSU02060 & 0.844 \\
\hline 35 & 2106333 & 2106489 & forward & BSU19330 & sodF & -1353 & 0.3885 & 79 & yocs & BSU19350 & 0.844 \\
\hline 36 & 308175 & 308331 & forward & BSU02840 & $y c d G$ & 48 & 0.3503 & 79 & $\operatorname{adc} A$ & BSU02850 & 0.843 \\
\hline 37 & 2678925 & 2679081 & forward & BSU26050 & yqdB & -427 & 0.363 & 12639 & yqaP & BSU26230 & 0.843 \\
\hline 38 & 3875571 & 3875727 & reverse & BSU37760 & rocC & -130 & 0.3121 & 79 & ywfA & BSU37750 & 0.843 \\
\hline 39 & 2433680 & 2433836 & reverse & BSU23340 & ypuB & -384 & 0.3885 & 236 & ypz」 & BSU23328 & 0.843 \\
\hline 40 & 1533806 & 1533962 & forward & BSU14610 & $\mathrm{pdhD}$ & -445 & 0.3503 & 236 & ykzW & BSU14629 & 0.841 \\
\hline 41 & 368137 & 368293 & forward & BSU03360 & yciC & -802 & 0.3312 & 1021 & yckC & BSU03390 & 0.841 \\
\hline 42 & 447000 & 447156 & forward & BSU03930 & gdh & -792 & 0.3121 & 2120 & $y c n L$ & BSU03970 & 0.84 \\
\hline 43 & 2065804 & 2065960 & forward & BSU18960 & yozM & -348 & 0.3758 & 8557 & yobN & BSU19020 & 0.839 \\
\hline 44 & 45296 & 45452 & reverse & BSU01550 & gerD & -113140 & 0.3822 & 236 & abrB & BSU00370 & 0.837 \\
\hline 45 & 2048779 & 2048935 & reverse & BSU18810 & yobA & -1092 & 0.363 & 550 & yoaZ & BSU18790 & 0.836 \\
\hline 46 & 3153718 & 3153874 & reverse & BSU30850 & $y t d A$ & -938 & 0.3439 & 79 & menF & BSU30830 & 0.836 \\
\hline 47 & 3388260 & 3388416 & reverse & BSU33040 & fumc & -685 & 0.3312 & 393 & yuzO & BSU33029 & 0.834 \\
\hline 48 & 205252 & 205408 & forward & BSU01820 & $\mathrm{adaB}$ & -283 & 0.3248 & 79 & ndhF & BSU01830 & 0.834 \\
\hline 49 & 469269 & 469425 & forward & BSU04160 & $m t \mid R$ & 24 & 0.3121 & 79 & $y d a B$ & BSU04170 & 0.834 \\
\hline 50 & 1868460 & 1868616 & forward & BSU17360 & $y m z A$ & -7 & 0.3885 & 79 & nrdl & BSU17370 & 0.834 \\
\hline 51 & 3746069 & 3746225 & forward & BSU36380 & rapD & -577 & 0.3822 & 2905 & ywoH & BSU36440 & 0.833 \\
\hline 52 & 3467327 & 3467483 & reverse & BSU33800 & opuCD & -140 & 0.3376 & 79 & $s d p R$ & BSU33790 & 0.832 \\
\hline 53 & 1903262 & 1903418 & reverse & BSU17690 & yncM & -170 & 0.3822 & 1963 & $\cot U$ & BSU17670 & 0.831 \\
\hline 54 & 1017114 & 1017270 & forward & BSU09400 & spoVR & 18 & 0.3376 & 1806 & lytE & BSU09420 & 0.831 \\
\hline 55 & 2709577 & 2709733 & reverse & BSU26490 & yrkJ & -346 & 0.3503 & 236 & yrkK & BSU26480 & 0.829 \\
\hline
\end{tabular}


Table 23 Top classification hits in B. subtilis uracil-comp. constrained (Continued)

\begin{tabular}{|c|c|c|c|c|c|c|c|c|c|c|c|}
\hline 56 & 955738 & 955894 & forward & BSU08780 & ygaJ & -74 & 0.3822 & 79 & thiC & BSU08790 & 0.828 \\
\hline 57 & 1283149 & 1283305 & forward & BSU12100 & yjeA & -539 & 0.3758 & 236 & yjfC & BSU12130 & 0.827 \\
\hline 58 & 3988764 & 3988920 & reverse & BSU38860 & galE & -1105 & 0.293 & 79 & yxkD & BSU38840 & 0.825 \\
\hline 59 & 200120 & 200276 & forward & BSU01770 & glmM & -198 & 0.2611 & 79 & $\mathrm{glmS}$ & BSU01780 & 0.825 \\
\hline 60 & 2186812 & 2186968 & reverse & BSU20420 & yorD & -94 & 0.2611 & 79 & yorE & BSU20410 & 0.825 \\
\hline 61 & 2926840 & 2926996 & reverse & BSU28630 & pheT & -89 & 0.3185 & 1021 & yshA & BSU28610 & 0.823 \\
\hline 62 & 2054401 & 2054557 & reverse & BSU18840 & xynA & -119 & 0.3822 & 550 & pps & BSU18830 & 0.822 \\
\hline 63 & 610963 & 611119 & reverse & BSU05660 & ydgl & -1149 & 0.3121 & 2277 & $\operatorname{din} B$ & BSU05630 & 0.822 \\
\hline 64 & 3457144 & 3457300 & reverse & BSU33700 & opuBD & -2583 & 0.3185 & 707 & $y v z C$ & BSU33650 & 0.821 \\
\hline 65 & 736435 & 736591 & reverse & BSU06740 & yefB & -2481 & 0.3376 & 3690 & yerO & BSU06700 & 0.82 \\
\hline 66 & 2061478 & 2061634 & reverse & BSU18930 & yobH & -1953 & 0.3439 & 864 & yozJ & BSU18900 & 0.82 \\
\hline 67 & 3268477 & 3268633 & forward & BSU31810 & yuzE & -4174 & 0.3949 & 8400 & yukF & BSU31920 & 0.82 \\
\hline 68 & 3107044 & 3107200 & forward & BSU30340 & ytvA & 30 & 0.2675 & 1492 & yttA & BSU30360 & 0.82 \\
\hline 69 & 2262616 & 2262772 & reverse & BSU21440 & $b d b B$ & -2530 & 0.3885 & 15151 & youB & BSU21329 & 0.819 \\
\hline 70 & 4118717 & 4118873 & reverse & BSU40110 & bglA & -2370 & 0.3758 & 5574 & glxk & BSU 40040 & 0.818 \\
\hline 71 & 4204755 & 4204911 & reverse & BSU40960 & parB & -722 & 0.3949 & 393 & yyaD & BSU40940 & 0.818 \\
\hline 72 & 252357 & 252513 & forward & BSU02320 & ybfP & 36 & 0.3822 & 79 & ybfQ & BSU02330 & 0.818 \\
\hline 73 & 3648264 & 3648420 & reverse & BSU35530 & $\operatorname{tagO}$ & -311 & 0.363 & 1806 & $\operatorname{deg} S$ & BSU35500 & 0.818 \\
\hline 74 & 850053 & 850209 & forward & BSU07750 & yflA & -3789 & 0.3694 & 236 & treP & BSU07800 & 0.817 \\
\hline 75 & 255279 & 255435 & forward & BSU02330 & ybfQ & -1718 & 0.2994 & 2434 & ybgA & BSU02370 & 0.816 \\
\hline 76 & 1541729 & 1541885 & forward & BSU14680 & $y k z C$ & -2958 & 0.3376 & 79 & ylaA & BSU14710 & 0.816 \\
\hline 77 & 909862 & 910018 & forward & BSU08330 & yfiN & -658 & 0.3503 & 79 & estB & BSU08350 & 0.816 \\
\hline 78 & 4109617 & 4109773 & reverse & BSU40030 & yxaB & -1253 & 0.3885 & 79 & $y x a D$ & BSU40010 & 0.813 \\
\hline 79 & 3252983 & 3253139 & forward & BSU31660 & mrpG & -538 & 0.3949 & 4632 & yuzC & BSU31730 & 0.811 \\
\hline 80 & 4066294 & 4066450 & reverse & BSU39600 & yxeC & -234 & 0.3567 & 864 & yxeF & BSU39570 & 0.81 \\
\hline 81 & 1923077 & 1923233 & forward & BSU17910 & yneF & -231 & 0.3248 & 79 & $\operatorname{ccdA}$ & BSU17930 & 0.809 \\
\hline 82 & 1540787 & 1540943 & forward & BSU14680 & $y k z C$ & -2016 & 0.3503 & 1021 & ylaA & BSU14710 & 0.809 \\
\hline 83 & 3665472 & 3665628 & forward & BSU35650 & lytR & -1192 & 0.3376 & 79 & gtaB & BSU35670 & 0.808 \\
\hline
\end{tabular}


Table 23 Top classification hits in B. subtilis uracil-comp. constrained (Continued)

\begin{tabular}{|c|c|c|c|c|c|c|c|c|c|c|c|}
\hline 84 & 1679031 & 1679187 & reverse & BSU17060 & ymzD & -101508 & 0.3503 & 7458 & ylqB & BSU15960 & 0.808 \\
\hline 85 & 2698717 & 2698873 & reverse & BSU26360 & yqaD & -714 & 0.363 & 79 & yqaF & BSU26340 & 0.808 \\
\hline 86 & 3604725 & 3604881 & reverse & BSU35100 & $y v \mid D$ & -1958 & 0.363 & 236 & yvmC & BSU35070 & 0.808 \\
\hline 87 & 3354671 & 3354827 & forward & BSU32650 & yurs & -105 & 0.3057 & 17820 & yuzL & BSU32849 & 0.807 \\
\hline 88 & 3052234 & 3052390 & forward & BSU29710 & acuC & -9600 & 0.3503 & 2434 & ytoQ & BSU29850 & 0.806 \\
\hline 89 & 188867 & 189023 & forward & BSU01590 & ybas & -9203 & 0.3185 & 5260 & trnSL-Glu2 & BSU_tRNA_75 & 0.806 \\
\hline 90 & 245389 & 245545 & reverse & BSU02340 & gltP & -8050 & 0.363 & 1806 & ybfl & BSU02220 & 0.805 \\
\hline 91 & 1445373 & 1445529 & reverse & BSU13810 & ykvs & -2210 & 0.3439 & 2748 & $y k v N$ & BSU13760 & 0.804 \\
\hline 92 & 2249114 & 2249270 & reverse & BSU21440 & $b d b B$ & -16032 & 0.3439 & 1649 & youB & BSU21329 & 0.803 \\
\hline 93 & 3918262 & 3918418 & reverse & BSU38190 & galT & -752 & 0.3057 & 79 & qoxA & BSU38170 & 0.801 \\
\hline 94 & 933760 & 933916 & reverse & BSU08620 & yfhP & -618 & 0.3439 & 5574 & sspK & BSU08550 & 0.8 \\
\hline 95 & 201248 & 201404 & reverse & BSU01800 & alkA & -1220 & 0.293 & 7301 & ybbk & BSU01720 & 0.8 \\
\hline 96 & 3684268 & 3684424 & reverse & BSU35780 & lytD & -479 & 0.3439 & 3376 & $\operatorname{tag} \mathrm{D}$ & BSU35740 & 0.8 \\
\hline 97 & 2739834 & 2739990 & reverse & BSU26830 & yrpE & -1444 & 0.3439 & 3376 & aadk & BSU26790 & 0.799 \\
\hline 98 & 2252097 & 2252253 & reverse & BSU21440 & $b d b B$ & -13049 & 0.3949 & 4632 & youB & BSU21329 & 0.798 \\
\hline 99 & 1601271 & 1601427 & reverse & BSU15640 & yloA & -34781 & 0.3503 & 24100 & ylbP & BSU15100 & 0.797 \\
\hline 100 & 2111609 & 2111765 & reverse & BSU19380 & yojO & -149 & 0.3439 & 79 & sucA & BSU19370 & 0.796 \\
\hline
\end{tabular}

Top 50 hits of the forward and reverse strands of the B. subtilis intergenic regions using no-overlap $157 \mathrm{nt}$ window and under the LMFEGCRND model. Uracil composition constrained to that of the range of known riboswitches in B. subtilis (between 0.2484 and 0.40127 ). The ranking of each hit is denoted in column R. Distance from upstream and downstream operons are the distance from the center of the hit to the stop and start codons of upstream and downstream operons, respectively. Probability denotes the multinomial regression likelihood of being a riboswitch under the LMFEGCRND model.

Interesting hits are shown in bold. 


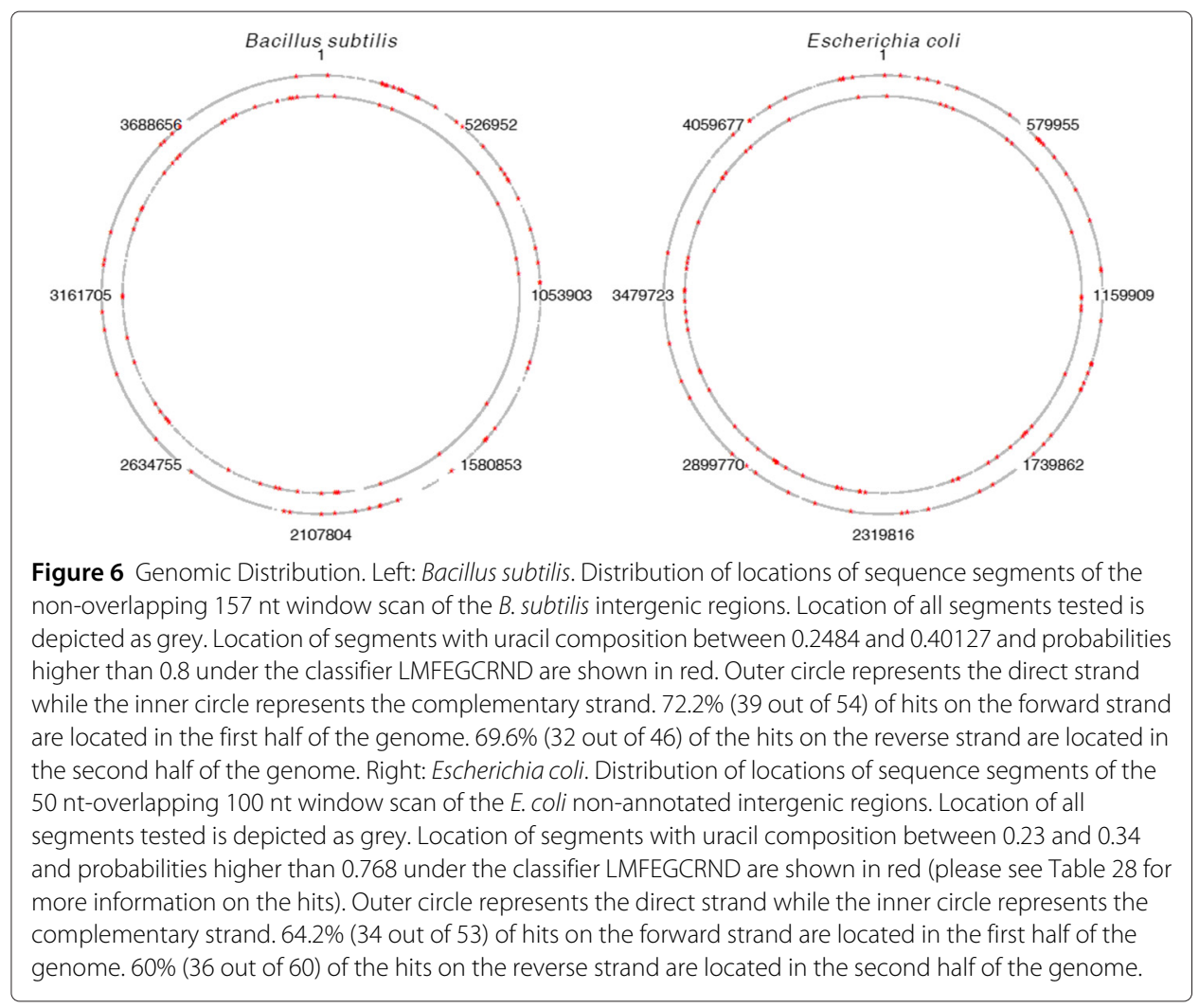


Table 24 Top entropy hits in B. subtilis filtered for GC-comp. and uracil-comp.

\begin{tabular}{|c|c|c|c|c|c|c|c|c|c|c|c|c|c|c|c|c|}
\hline B. subtilis & Start & End & Strand & Upstream operon & $\begin{array}{l}\text { Upstream } \\
\text { gene }\end{array}$ & $\begin{array}{l}\text { Dist. to } \\
\text { upstream }\end{array}$ & MFE & MFE p. Val. & $\mathrm{GC}$ & RND & RND p. Val. & Uracil & $\begin{array}{l}\text { Dist. to } \\
\text { downstream }\end{array}$ & $\begin{array}{l}\text { Downstream } \\
\text { gene }\end{array}$ & $\begin{array}{l}\text { Downstream } \\
\text { operon }\end{array}$ & Probability \\
\hline $157 \mathrm{nt}$ & 191850 & 192006 & forward & BSU01590 & ybas & -12186 & -54.16 & 0.01 & 0.4904 & 94.7470016 & 0.0359 & 0.3057 & 2277 & trnSL-Glu2 & BSU_tRNA_75 & 0.8561704159 \\
\hline $157 \mathrm{nt}$ & 749147 & 749303 & forward & BSU06780 & yeec & -3069 & -49.19 & - & 0.4458 & 94.8936005 & 0.0310 & 0.3630 & 550 & yeeG & BSU06820 & 0.8463344574 \\
\hline $157 \mathrm{nt}$ & 665425 & 665581 & forward & BSU06130 & ydjc & -677 & -51.50 & - & 0.4968 & 95.6813965 & 0.0169 & 0.3439 & 1963 & gutB & BSU06150 & 0.8462108970 \\
\hline $157 \mathrm{nt}$ & 1017114 & 1017270 & forward & BSU09400 & spoVR & 18 & -53.10 & - & 0.4968 & 94.5084991 & 0.0412 & 0.3376 & 1806 & lytE & BSU09420 & 0.8305525184 \\
\hline $157 \mathrm{nt}$ & 823013 & 823169 & forward & BSU07480 & yfmG & -604 & -48.60 & - & 0.5032 & 94.2139969 & 0.0507 & 0.2866 & 4161 & yfmA & BSU07540 & 0.7458834648 \\
\hline $157 \mathrm{nt}$ & 3421066 & 3421222 & reverse & BSU33340 & ssp」 & -320 & -49.40 & - & 0.4713 & 97.1984024 & 0.0049 & 0.3312 & 79 & lysP & BSU33330 & 0.8851321340 \\
\hline $157 \mathrm{nt}$ & 3158851 & 3159007 & reverse & BSU30890 & $y t x O$ & -328 & -48.50 & - & 0.4395 & 95.1657028 & 0.0250 & 0.3630 & 3376 & ytdA & BSU30850 & 0.8522043228 \\
\hline $157 \mathrm{nt}$ & 736435 & 736591 & reverse & BSU06740 & yefB & -2481 & -50.51 & - & 0.4904 & 95.0205994 & 0.0279 & 0.3376 & 3690 & yerO & BSU06700 & 0.8204180002 \\
\hline $157 \mathrm{nt}$ & 201248 & 201404 & reverse & BSU01800 & alkA & -1220 & -49.46 & - & 0.4968 & 95.0683975 & 0.0269 & 0.2930 & 7301 & ybbk & BSU01720 & 0.8003951907 \\
\hline $157 \mathrm{nt}$ & 4129689 & 4129845 & reverse & BSU40200 & yydD & -810 & -48.40 & - & 0.4904 & 94.8125000 & 0.0332 & 0.3567 & 2120 & yydF & BSU40180 & 0.7834032774 \\
\hline $150 \mathrm{nt}$ & 4134601 & 4134750 & forward & BSU40190 & $\mathrm{fbp}$ & -4483 & -50.91 & - & 0.4733 & 91.2214966 & - & 0.3800 & 677 & yycs & BSU40240 & 0.8779885173 \\
\hline $150 \mathrm{nt}$ & 3359819 & 3359968 & forward & BSU32650 & yurs & -5258 & -46.80 & - & 0.4600 & 92.3918991 & - & 0.3600 & 12677 & yuzL & BSU32849 & 0.8770275712 \\
\hline $150 \mathrm{nt}$ & 49175 & 749324 & forwa & BSU06780 & yeeC & -3102 & -46.50 & - & 0.4600 & 92.0363998 & - & 0.3867 & 527 & yeeG & BSU06820 & 0.8652582169 \\
\hline $150 \mathrm{nt}$ & 1958237 & 1958386 & forward & BSU18190 & yngC & -9899 & -48.30 & - & 0.4733 & 90.9682007 & - & 0.3533 & 44327 & iseA & BSU18380 & 0.8436317444 \\
\hline $150 \mathrm{nt}$ & 1540761 & 1540910 & forward & BSU14680 & ykzC & -1995 & -46.42 & & 0.4333 & 90.3455963 & - & 0.3400 & 1052 & ylaA & BSU14710 & 0.8428211212 \\
\hline $150 \mathrm{nt}$ & 3199841 & 3199990 & forward & BSU31170 & yulF & -1875 & -47.20 & - & 0.4467 & 89.9530029 & - & 0.3733 & 12677 & $\operatorname{tgl}$ & BSU31270 & 0.8267914653 \\
\hline $150 \mathrm{nt}$ & 3421066 & 3421215 & reverse & BSU33340 & sspJ & -325 & -49.40 & - & 0.4800 & 93.4540024 & - & 0.3333 & 74 & lysP & BSU33330 & 0.9072541595 \\
\hline $150 \mathrm{nt}$ & 933665 & 933814 & reverse & BSU08620 & yfhP & -718 & -49.54 & - & 0.4600 & 89.9813995 & - & 0.3733 & 5474 & sspK & BSU08550 & 0.8426564932 \\
\hline 200 nt & 3359769 & 3359968 & forward & BSU32650 & yurs & -5183 & -66.40 & - & 0.4600 & 123.4530029 & - & 0.3450 & 12702 & yuzL & BSU32849 & 0.9236087203 \\
\hline $200 \mathrm{nt}$ & 339225 & 339424 & forward & BSU03130 & nadE & & -63.60 & & 0.4700 & & - & & & & & \\
\hline $200 \mathrm{nt}$ & 1678852 & 1679051 & reverse & BSU17060 & ymzD & -101667 & -62.81 & & 0.4750 & 122.0299988 & - & 0.3300 & 7299 & ylqB & BSU15960 & 0.8517054319 \\
\hline $200 \mathrm{nt}$ & 3717398 & 3717597 & reverse & BSU36100 & ywrD & -1637 & -51. & & 0.3650 & 130.8540039 & & 0.3950 & 399 & & & 0.9702541828 \\
\hline & & 198425 & reverse & BSU01800 & alkA & -4222 & -30.81 & & 0.3750 & 130.7449951 & - & 0.5150 & 4299 & ybbk & BSU01720 & 0.8267450333 \\
\hline $157 \mathrm{nt}$ & 235800 & 235956 & reverse & BSU02180 & ybfE & -2285 & -54.99 & - & 0.3312 & 66.4815979 & $0^{1}$ & 0.3439 & 550 & glpT & BSU02140 & 0.0401644297 \\
\hline $200 \mathrm{nt}$ & 3236257 & 3236456 & forward & BSU31500 & yuxk & 61 & -82.70 & - & 0.4200 & 93.3933029 & $0^{2}$ & 0.2650 & 802 & yufL & BSU31520 & 0.0853443071 \\
\hline
\end{tabular}

Significant hits of the forward and reverse strands of the B. subtilis intergenic regions having significantly high RND entropy (p-Val. < 0.0500), significantly low (p.Val. $<0.050)$, GC and uracil compositions within the range of those for known riboswitches Threshold values and their corresponding p-values have been calculated separately for each genome-wide test. No overlap used for $157 \mathrm{nt}$ scan ( 28340 segments). $175 \mathrm{nt}$ overlap used for $150 \mathrm{nt}$ scan (60204 segments). $100 \mathrm{nt}$ overlap used for $200 \mathrm{nt}$ scan (44847 segments). Distance from upstream and downstream operons are the distance from the center of the hit to the stop and start codons of upstream and downstream operons, respectively. Probability denotes the multinomial regression likelihood of being a riboswitch under the LMFEGCRND model. Negative values indicate distance to upstream operon. Columns Upsream/Downstream Operon show gene ID within the operon.

${ }^{1}$ Table 24: The entropy of this sequence is the lowest within the test. The significance of this value is also shown in Figure 5 as the lowest blue point on the graph.

${ }^{2}$ Table 24: The entropy of this sequence is the lowest within the test. 
Table 25 Top entropy hits in B. subtilis forward strand

\begin{tabular}{|c|c|c|c|c|c|c|c|c|c|c|c|c|c|c|}
\hline B. subtilis & Start & End & Strand Upstream operon & $\begin{array}{l}\text { Upstream } \\
\text { gene }\end{array}$ & $\begin{array}{l}\text { Dist. to } \\
\text { upstream }\end{array}$ & MFE & MFE p. Val. C & GC & RND & RND p. Val. Uracil & $\begin{array}{l}\text { Dist. to } \\
\text { downstream }\end{array}$ & $\begin{array}{l}\text { Downstream } \\
\text { gene }\end{array}$ & $\begin{array}{l}\text { Downstream } \\
\text { operon }\end{array}$ & Probability \\
\hline $200 \mathrm{nt}$ & 3714838 & 3715037 & forward BSU36030 & ywrk & -794 & -49.70 & - & 0.3300 & 126.0619965 & 0.3850 & 2302 & $\cot G$ & BSU36070 & 0.9275143743 \\
\hline $200 \mathrm{nt}$ & 3359769 & 3359968 & forward BSU32650 & yurs & -5183 & -66.40 & - & 0.4600 & 123.4530029 & 0.3450 & 12702 & yuzL & BSU32849 & 0.9236087203 \\
\hline $200 \mathrm{nt}$ & 243592 & 243791 & forward BSU02170 & $y b f B$ & -5364 & -57.00 & - & 0.4450 & 126.5479965 & 0.3700 & 202 & purT & BSU02230 & 0.9204539061 \\
\hline $200 \mathrm{nt}$ & 2093202 & 2093401 & forward BSU19200 & desR & -799 & -55.20 & - & 0.4350 & 126.5859985 & 0.4400 & 4802 & уоув & BSU19259 & 0.9146069884 \\
\hline $200 \mathrm{nt}$ & 749075 & 749274 & forward BSU06780 & yeec & -2977 & -58.19 & - & 0.4450 & 125.6159973 & 0.3900 & 602 & yeeG & BSU06820 & 0.9128865004 \\
\hline $200 \mathrm{nt}$ & 1467005 & 1467204 & forward BSU13960 & ykwC & -307 & -60.40 & - & 0.4100 & 123.2139969 & 0.4150 & 702 & $\mathrm{pbpH}$ & BSU13980 & 0.9070840478 \\
\hline $200 \mathrm{nt}$ & 2281367 & 2281566 & forward BSU21620 & yokE & 95 & -44.60 & - & 0.2600 & 124.4599991 & 0.4300 & 202 & yokD & BSU21630 & 0.9058990479 \\
\hline $200 \mathrm{nt}$ & 850067 & 850266 & forward BSU07750 & yflA & -3783 & -57.20 & - & 0.4000 & 124.1029968 & 0.3800 & 202 & treP & BSU07800 & 0.9058393836 \\
\hline $200 \mathrm{nt}$ & 1466905 & 1467104 & forward BSU13960 & ykwC & -207 & -56.93 & - & 0.3900 & 123.7220001 & 0.4200 & 802 & $\mathrm{pbpH}$ & BSU13980 & 0.9029595852 \\
\hline $200 \mathrm{nt}$ & 3759694 & 3759893 & forward BSU36530 & bcrc & -467 & -62.40 & - & 0.3850 & 121.3089981 & 0.4500 & 902 & ywnH & BSU36560 & 0.9023656249 \\
\hline $200 \mathrm{nt}$ & 3268355 & 3268554 & forward BSU31810 & yuzE & -4032 & -44.66 & - & 0.3750 & 127.7630005 & 0.4450 & 8502 & yukF & BSU31920 & 0.8946693540 \\
\hline $200 \mathrm{nt}$ & 2073039 & 2073238 & forward BSU18960 & yozM & -7563 & -52.00 & - & 0.3250 & 123.0869980 & 0.4450 & 1302 & yobN & BSU19020 & 0.8944090009 \\
\hline $200 \mathrm{nt}$ & 748975 & 749174 & forward BSU06780 & yeec & -2877 & -58.80 & - & 0.4750 & 125.1039963 & 0.3750 & 702 & yeeG & BSU06820 & 0.8876969814 \\
\hline $200 \mathrm{nt}$ & 432172 & 432371 & forward BSU03780 & phrc & -1988 & -51.70 & - & 0.3050 & 122.0960007 & 0.3450 & 102 & yclN & BSU03800 & 0.8850299716 \\
\hline $200 \mathrm{nt}$ & 4039583 & 4039782 & forward BSU39100 & yxiO & -23516 & -53.00 & - & 0.4350 & 125.9430008 & 0.4350 & 1802 & hutP & BSU39340 & 0.8843178153 \\
\hline $200 \mathrm{nt}$ & 531587 & 531786 & forward BSU_tRNA_51 & trnS-Leu2 & -2066 & -46.60 & - & 0.2650 & 122.5979996 & 0.3850 & 102 & sacV & BSU04830 & 0.8803396225 \\
\hline $200 \mathrm{nt}$ & 665366 & 665565 & forward BSU06130 & ydjc & -598 & -64.50 & - & 0.4900 & 122.9229965 & 0.3300 & 2002 & gutB & BSU06150 & 0.8790112138 \\
\hline $200 \mathrm{nt}$ & 3714938 & 3715137 & forward BSU36030 & ywrk & -894 & -41.70 & - & 0.3350 & 126.8040009 & 0.3550 & 2202 & $\cot G$ & BSU36070 & 0.8768669963 \\
\hline $200 \mathrm{nt}$ & 3685812 & 3686011 & forward BSU35770 & $\operatorname{tag} C$ & -947 & -51.80 & - & 0.3900 & 124.4950027 & 0.4150 & 2902 & gerBA & BSU35800 & 0.8747953176 \\
\hline $200 \mathrm{nt}$ & 2093302 & 2093501 & forward BSU19200 & desR & -899 & -61.40 & - & 0.4400 & 122.2649994 & 0.3700 & 4702 & уоуB & BSU19259 & 0.8738172650 \\
\hline $200 \mathrm{nt}$ & 1012447 & 1012646 & forward BSU09360 & yhdC & -598 & -51.20 & - & 0.3650 & 123.8079987 & 0.4100 & 3102 & spoVR & BSU09400 & 0.8727893829 \\
\hline $200 \mathrm{nt}$ & 955595 & 955794 & forward BSU08780 & ygaJ & 89 & -59.79 & - & 0.4050 & 121.6190033 & 0.3600 & 202 & thic & BSU08790 & 0.8710888028 \\
\hline $200 \mathrm{nt}$ & 3685912 & 3686111 & forward BSU35770 & $\operatorname{tag} C$ & -1047 & -53.60 & - & 0.3950 & 123.7610016 & 0.3950 & 2802 & gerBA & BSU35800 & 0.8705116510 \\
\hline $200 \mathrm{nt}$ & 4134651 & 4134850 & forward BSU40190 & $\mathrm{fbp}$ & -4508 & -58.20 & - & 0.4900 & 125.0049973 & 0.3900 & 602 & yycs & BSU40240 & 0.8676616549 \\
\hline $200 \mathrm{nt}$ & 45433 & 45632 & forward BSU00360 & yabC & -535 & -42.70 & - & 0.3000 & 124.7969971 & 0.4200 & 102 & mets & BSU00380 & 0.8666248322 \\
\hline $200 \mathrm{nt}$ & 2531951 & 2532150 & forward BSU24210 & yqiG & -14294 & -62.90 & - & 0.5050 & 123.56999997 & 0.3450 & 9002 & yqhe & BSU24490 & 0.8666005135 \\
\hline
\end{tabular}




\section{Table 25 Top entropy hits in B. subtilis forward strand (Continued)}

\begin{tabular}{|c|c|c|c|c|c|c|c|c|c|c|c|c|c|c|}
\hline $200 \mathrm{nt}$ & 2531951 & 2532150 & forward & BSU24210 & yqiG & -14294 & -62.90 & - 0.5050 & 123.5699997 & 0.3450 & 9002 & yqhe & BSU24490 & 0.8666005135 \\
\hline $200 \mathrm{nt}$ & 1540786 & 1540985 & forward & BSU14680 & $y k z C$ & -1995 & -57.52 & - 0.4350 & 123.3629990 & 0.3750 & 1002 & ylaA & BSU14710 & 0.8663020134 \\
\hline $200 \mathrm{nt}$ & 1406312 & 1406511 & forward & BSU13390 & ykoT & -1721 & -71.55 & - 0.5450 & 121.3809967 & 0.3250 & 3502 & ykoX & BSU13430 & 0.8654530644 \\
\hline $200 \mathrm{nt}$ & 4029283 & 4029482 & forward & BSU39100 & yxiO & -13216 & -50.90 & -0.3850 & 124.3079987 & 0.4000 & 12102 & hutP & BSU39340 & 0.8653051257 \\
\hline $200 \mathrm{nt}$ & 1526531 & 1526730 & forward & BSU14550 & ykrA & -273 & -63.20 & - 0.4800 & 122.5350037 & 0.3350 & 602 & ykyA & BSU14570 & 0.8648978472 \\
\hline $200 \mathrm{nt}$ & 2220040 & 2220239 & forward & BSU20929 & yoyl & -6863 & -38.72 & - 0.3800 & 129.0460052 & 0.3700 & 2202 & yonP & BSU21030 & 0.8647925854 \\
\hline $200 \mathrm{nt}$ & 192105 & 192304 & forward & BSU01590 & ybas & -12421 & -51.20 & - 0.4350 & 125.8399963 & 0.4100 & 2002 & $\operatorname{trnSL-Glu2}$ & BSU_tRNA_75 & 0.8642573953 \\
\hline $200 \mathrm{nt}$ & 1780406 & 1780605 & forward & BSU17050 & mutL & -87 & -52.60 & - 0.3550 & 122.5059967 & 0.3650 & 1402 & pksA & BSU17080 & 0.8627771139 \\
\hline $200 \mathrm{nt}$ & 4037783 & 4037982 & forward & BSU39100 & yxiO & -21716 & -57.74 & -0.4400 & 123.2249985 & 0.3950 & 3602 & hutP & BSU39340 & 0.8606380820 \\
\hline $200 \mathrm{nt}$ & 1264357 & 1264556 & forward & BSU_tRNA_83 & $\operatorname{trnSL-Val2}$ & -1397 & -33.05 & - $\quad 0.2700$ & 127.4010010 & 0.4200 & 602 & yjcN & BSU11920 & 0.8592507839 \\
\hline $200 \mathrm{nt}$ & 847767 & 847966 & forward & BSU07750 & yflA & -1483 & -59.62 & - $\quad 0.4500$ & 122.6080017 & 0.3550 & 2502 & treP & BSU07800 & 0.8554052114 \\
\hline $200 \mathrm{nt}$ & 226266 & 226465 & forward & BSU02050 & ybdO & 81 & -37.20 & - 0.2300 & 124.2030029 & 0.3550 & 202 & ybxG & BSU02060 & 0.8548350334 \\
\hline $200 \mathrm{nt}$ & 3052246 & 3052445 & forward & BSU29710 & acuC & -9592 & -61.90 & - 0.4900 & 123.0039978 & 0.3400 & 2402 & ytoQ & BSU29850 & 0.8543979526 \\
\hline $200 \mathrm{nt}$ & 530887 & 531086 & forward & BSU_tRNA_51 & trnS-Leu2 & -1366 & -42.20 & - 0.3250 & 125.3190002 & 0.4000 & 802 & sacV & BSU04830 & 0.8526363969 \\
\hline $200 \mathrm{nt}$ & 2617117 & 2617316 & forward & BSU25220 & antE & -13743 & -59.90 & - 0.4500 & 122.3389969 & 0.4200 & 3502 & yqeW & BSU25420 & 0.8511158824 \\
\hline $200 \mathrm{nt}$ & 2221540 & 2221739 & forward & BSU20929 & yoyl & -8363 & -46.30 & $-\quad 0.2850$ & 122.2210007 & 0.3450 & 702 & yonP & BSU21030 & 0.8502966762 \\
\hline $200 \mathrm{nt}$ & 2054178 & 2054377 & forward & BSU18820 & yobB & -3127 & -48.70 & - 0.3450 & 123.2819977 & 0.4000 & 2002 & yobD & BSU18850 & 0.8502687216 \\
\hline $200 \mathrm{nt}$ & 3042946 & 3043145 & forward & BSU29710 & acuC & -292 & -49.94 & - 0.4350 & 125.8190002 & 0.4150 & 11702 & ytoQ & BSU29850 & 0.8499680161 \\
\hline $200 \mathrm{nt}$ & 2780909 & 2781108 & forward & BSU27150 & yrhK & -7164 & -47.80 & - 0.3250 & 122.8980026 & 0.3750 & 202 & yrhE & BSU27220 & 0.8482846022 \\
\hline $200 \mathrm{nt}$ & 2723337 & 2723536 & forward & BSU26630 & yrdQ & -594 & -44.00 & - 0.2950 & 123.3580017 & - 0.4300 & 2402 & gltR & BSU26670 & 0.8465546370 \\
\hline $200 \mathrm{nt}$ & 683762 & 683961 & forward & BSU06260 & ydjN & -2790 & -59.40 & - $\quad 0.4800$ & 123.3310013 & - $\quad 0.3600$ & 3102 & yeaB & BSU06320 & 0.8446838856 \\
\hline $200 \mathrm{nt}$ & 3052346 & 3052545 & forward & BSU29710 & acuc & -9692 & -56.62 & - 0.4400 & 123.0849991 & 0.3800 & 2302 & ytoQ & BSU29850 & 0.8442399502 \\
\hline $200 \mathrm{nt}$ & 2405829 & 2406028 & forward & BSU22869 & ypzl & -12171 & -60.30 & - 0.4650 & 122.4049988 & 0.4150 & 3802 & fer & BSU23040 & 0.8431282043 \\
\hline $200 \mathrm{nt}$ & 579341 & 579540 & forward & BSU05329 & $y d z O$ & -10 & -47.40 & - 0.3850 & 124.9130020 & 0.3050 & 102 & aseR & BSU05330 & 0.8431255221 \\
\hline $200 \mathrm{nt}$ & 748875 & 749074 & forward & BSU06780 & yeeC & -2777 & -60.60 & - 0.4600 & 122.0989990 & 0.3950 & 802 & yeeG & BSU06820 & 0.8426845670 \\
\hline $200 \mathrm{nt}$ & 339225 & 339424 & forward & BSU03130 & nadE & -20 & -63.60 & - 0.4700 & 121.1809998 & 0.3250 & 702 & arok & BSU03150 & 0.8414211273 \\
\hline $200 \mathrm{nt}$ & 1251377 & 1251576 & forward & BSU11730 & cotO & -1930 & -35.49 & - 0.3200 & 127.4260025 & 0.4800 & 702 & yjcA & BSU11790 & 0.8401102424 \\
\hline $200 \mathrm{nt}$ & 3640353 & 3640552 & forward & BSU35210 & yvkA & -19956 & -52.60 & - 0.3600 & 121.8539963 & - $\quad 0.4250$ & 6302 & yvyE & BSU35510 & 0.8399478197 \\
\hline $200 \mathrm{nt}$ & 3686112 & 3686311 & forward & BSU35770 & $\operatorname{tag} C$ & -1247 & -51.99 & - $\quad 0.3750$ & 122.5879974 & - $\quad 0.4300$ & 2602 & gerBA & BSU35800 & 0.8393257856 \\
\hline
\end{tabular}


Table 25 Top entropy hits in B. subtilis forward strand (Continued)

\begin{tabular}{|c|c|c|c|c|c|c|c|c|c|c|c|c|c|c|c|}
\hline $200 \mathrm{nt}$ & 3686112 & 3686311 & forward & BSU35770 & $\operatorname{tag} C$ & -1247 & -51.99 & - & 0.3750 & 122.5879974 & 0.4300 & 2602 & gerBA & BSU35800 & 0.8393257856 \\
\hline $200 \mathrm{nt}$ & 1494405 & 1494604 & forward & BSU14240 & rok & 56 & -52.70 & - & 0.4100 & 123.4729996 & 0.4150 & 1002 & mobA & BSU14260 & 0.8389207721 \\
\hline $200 \mathrm{nt}$ & 373532 & 373731 & forward & BSU03410 & bglc & -1741 & -56.90 & - & 0.4500 & 123.1060028 & 0.3800 & 2402 & hxIR & BSU03470 & 0.8382304311 \\
\hline $200 \mathrm{nt}$ & 3686212 & 3686411 & forward & BSU35770 & $\operatorname{tag} C$ & -1347 & -45.70 & - & 0.4000 & 125.9280014 & 0.4250 & 2502 & gerBA & BSU35800 & 0.8377878666 \\
\hline $200 \mathrm{nt}$ & 374532 & 374731 & forward & BSU03410 & bglC & -2741 & -56.99 & - & 0.3750 & 120.5049973 & 0.3400 & 1402 & $h \times \mid R$ & BSU03470 & 0.8375294805 \\
\hline $200 \mathrm{nt}$ & 1540186 & 1540385 & forward & BSU14680 & $y k z C$ & -1395 & -61.11 & - & 0.4650 & 121.7969971 & 0.3500 & 1602 & ylaA & BSU14710 & 0.8347978592 \\
\hline $200 \mathrm{nt}$ & 360837 & 361036 & forward & BSU03270 & yсg & -6870 & -59.70 & - & 0.5000 & 123.5510025 & 0.3300 & 2002 & nas A & BSU03330 & 0.8347288966 \\
\hline $200 \mathrm{nt}$ & 213641 & 213840 & forward & BSU01900 & ybcM & -73 & -36.30 & - & 0.2750 & 125.3079987 & 0.3950 & 202 & skfA & BSU01910 & 0.8321032524 \\
\hline $200 \mathrm{nt}$ & 739678 & 739877 & forward & BSU06730 & yefA & -597 & -51.16 & - & 0.3900 & 123.1309967 & 0.4150 & 102 & yefC & BSU06750 & 0.8301935792 \\
\hline $200 \mathrm{nt}$ & 1495005 & 1495204 & forward & BSU14240 & rok & -544 & -50.26 & - & 0.4150 & 124.2959976 & 0.3800 & 402 & mobA & BSU14260 & 0.8287579417 \\
\hline $200 \mathrm{nt}$ & 1541686 & 1541885 & forward & BSU14680 & $y k z C$ & -2895 & -43.10 & - & 0.3250 & 124.1309967 & 0.3650 & 102 & ylaA & BSU14710 & 0.8283772469 \\
\hline $200 \mathrm{nt}$ & 1268629 & 1268828 & forward & BSU11970 & yjcS & 62 & -40.70 & - & 0.2700 & 123.2060013 & 0.4250 & 102 & yjdA & BSU11980 & 0.8273611665 \\
\hline $200 \mathrm{nt}$ & 652232 & 652431 & forward & BSU06030 & groEL & -265 & -37.70 & - & 0.2950 & 125.2659988 & 0.4500 & 1102 & ydiM & BSU06040 & 0.8273396492 \\
\hline $200 \mathrm{nt}$ & 2108093 & 2108292 & forward & BSU19350 & yocs & -539 & -59.80 & - & 0.4700 & 122.2429962 & 0.3700 & 11202 & yojl & BSU19440 & 0.8269666433 \\
\hline $200 \mathrm{nt}$ & 728532 & 728731 & forward & BSU06640 & yerl & -2436 & -46.30 & - & 0.3100 & 122.2779999 & 0.3600 & 102 & gatC & BSU06670 & 0.8268005848 \\
\hline $200 \mathrm{nt}$ & 1540686 & 1540885 & forward & BSU14680 & $y k z C$ & -1895 & -60.72 & - & 0.4350 & 120.6729965 & 0.3350 & 1102 & ylaA & BSU14710 & 0.8265900016 \\
\hline $200 \mathrm{nt}$ & 3746052 & 3746251 & forward & BSU36380 & rapD & -540 & -57.10 & - & 0.4350 & 122.1200027 & 0.3850 & 2902 & ywoH & BSU36440 & 0.8260388970 \\
\hline $200 \mathrm{nt}$ & 1495105 & 1495304 & forward & BSU14240 & rok & -644 & -55.30 & - & 0.3950 & 121.4899979 & 0.4000 & 302 & mobA & BSU14260 & 0.8259468675 \\
\hline $200 \mathrm{nt}$ & 1923034 & 1923233 & forward & BSU17910 & yneF & -168 & -40.60 & - & 0.2500 & 122.5039978 & 0.3500 & 102 & $\mathrm{ccdA}$ & BSU17930 & 0.8253148198 \\
\hline $200 \mathrm{nt}$ & 746475 & 746674 & forward & BSU06780 & yeec & -377 & -31.51 & - & 0.2650 & 126.6760025 & 0.5000 & 3202 & yeeG & BSU06820 & 0.8248795867 \\
\hline $200 \mathrm{nt}$ & 2625315 & 2625514 & forward & BSU25420 & yqeW & -3576 & -54.30 & - & 0.4850 & 124.8850021 & 0.3550 & 10402 & rpsT & BSU25550 & 0.8240758777 \\
\hline $200 \mathrm{nt}$ & 4007404 & 4007603 & forward & BSU39020 & $y x j A$ & -360 & -48.97 & - & 0.4150 & 124.6660004 & 0.3950 & 2902 & $\mathrm{citH}$ & BSU39060 & 0.8239642382 \\
\hline $200 \mathrm{nt}$ & 2376722 & 2376921 & forward & BSU22510 & ypjC & -15121 & -44.40 & - & 0.3450 & 124.1429977 & 0.4000 & 16602 & ypzl & BSU22869 & 0.8239628077 \\
\hline $200 \mathrm{nt}$ & 3640453 & 3640652 & forward & BSU35210 & yvkA & -20056 & -45.52 & - & 0.3450 & 123.6029968 & 0.4400 & 6202 & yvyE & BSU35510 & 0.8211722970 \\
\hline $200 \mathrm{nt}$ & 530787 & 530986 & forward & BSU_tRNA_51 & trnS-Leu2 & -1266 & -47.10 & - & 0.3350 & 122.6100006 & 0.3850 & 902 & sacV & BSU04830 & 0.8206871748 \\
\hline $200 \mathrm{nt}$ & 4139260 & 4139459 & forward & BSU40240 & yycs & -3397 & -60.10 & - & 0.5050 & 123.1039963 & 0.3450 & 902 & rapG & BSU40300 & 0.8204663396 \\
\hline $200 \mathrm{nt}$ & 184305 & 184504 & forward & BSU01590 & ybas & -4621 & -44.20 & - & 0.4000 & 125.9649963 & 0.4400 & 9802 & trnSL-Glu2 & BSU_tRNA_75 & 0.8200225234 \\
\hline $200 \mathrm{nt}$ & 792182 & 792381 & forward & BSU07230 & yetM & -656 & -69.00 & - & 0.5400 & 120.5859985 & 0.3000 & 402 & yetO & BSU07250 & 0.8170907497 \\
\hline $200 \mathrm{nt}$ & 4007304 & 4007503 & forward & BSU39020 & yxjA & -260 & -49.30 & - & 0.4200 & 124.4300003 & -0.3950 & 3002 & $\mathrm{citH}$ & BSU39060 & 0.8151187301 \\
\hline
\end{tabular}


Table 25 Top entropy hits in B. subtilis forward strand (Continued)

\begin{tabular}{|c|c|c|c|c|c|c|c|c|c|c|c|c|c|c|c|c|}
\hline $200 \mathrm{nt}$ & 3726352 & 3726551 & forward & BSU36160 & ywqM & -1918 & -63.90 & - & 0.5250 & 122.0380020 & - & 0.3200 & 7402 & $y w q B$ & BSU36270 & 0.8136813045 \\
\hline $200 \mathrm{nt}$ & 3201391 & 3201590 & forward & BSU31170 & yulf & -3400 & -50.17 & - & 0.4150 & 123.8629990 & - & 0.3950 & 11102 & $\operatorname{tgl}$ & BSU31270 & 0.8136008978 \\
\hline $200 \mathrm{nt}$ & 3714738 & 3714937 & forward & BSU36030 & ywrk & -694 & -38.82 & - & 0.3050 & 124.7269974 & - & 0.4300 & 2402 & $\cot G$ & BSU36070 & 0.8135811090 \\
\hline $200 \mathrm{nt}$ & 2160707 & 2160906 & forward & BSU20000 & yosU & -1924 & -34.70 & - & 0.3050 & 126.3850021 & - & 0.4300 & 9002 & yosA & BSU20190 & 0.8132891059 \\
\hline $200 \mathrm{nt}$ & 192805 & 193004 & forward & BSU01590 & ybas & -13121 & -48.80 & - & 0.4150 & 124.4029999 & - & 0.3650 & 1302 & trnSL-Glu2 & BSU_tRNA_75 & 0.8131257892 \\
\hline $200 \mathrm{nt}$ & 2217640 & 2217839 & forward & BSU20929 & yoyl & -4463 & -44.10 & - & 0.2800 & 121.7109985 & - & 0.4100 & 4602 & yonP & BSU21030 & 0.8124790788 \\
\hline $200 \mathrm{nt}$ & 182405 & 182604 & forward & BSU01590 & ybas & -2721 & -53.80 & - & 0.4150 & 122.3399963 & - & 0.3550 & 11702 & trnSL-Glu2 & BSU_tRNA_75 & 0.8117757440 \\
\hline $200 \mathrm{nt}$ & 2276877 & 2277076 & forward & BSU21520 & yolc & -4287 & -38.90 & - & 0.3250 & 125.3180008 & - & 0.4500 & 3002 & yokF & BSU21610 & 0.8117634654 \\
\hline $200 \mathrm{nt}$ & 1997137 & 1997336 & forward & BSU18190 & yngC & -48774 & -50.97 & - & 0.4000 & 122.9609985 & - & 0.3250 & 5402 & iseA & BSU18380 & 0.8112495542 \\
\hline $200 \mathrm{nt}$ & 2276977 & 2277176 & forward & BSU21520 & yolc & -4387 & -39.30 & - & 0.3200 & 124.9540024 & - & 0.4200 & 2902 & yokF & BSU21610 & 0.8106592894 \\
\hline $200 \mathrm{nt}$ & 749175 & 749374 & forward & BSU06780 & yeec & -3077 & -58.49 & - & 0.4600 & 121.9140015 & - & 0.3700 & 502 & yeeG & BSU06820 & 0.8099753261 \\
\hline $200 \mathrm{nt}$ & 3726652 & 3726851 & forward & BSU36160 & ywqM & -2218 & -52.11 & - & 0.4550 & 124.3000031 & - & 0.3950 & 7102 & $y w q B$ & BSU36270 & 0.8091073036 \\
\hline $200 \mathrm{nt}$ & 1251277 & 1251476 & forward & BSU11730 & $\operatorname{cotO}$ & -1830 & -37.50 & - & 0.3650 & 127.1539993 & - & 0.5150 & 802 & yjcA & BSU11790 & 0.8088676929 \\
\hline $200 \mathrm{nt}$ & 1405212 & 1405411 & forward & BSU13390 & ykoT & -621 & -56.26 & - & 0.4050 & 120.9140015 & - & 0.3500 & 4602 & ykoX & BSU13430 & 0.8086636066 \\
\hline $200 \mathrm{nt}$ & 3268455 & 3268654 & forward & BSU31810 & yuzE & -4132 & -52.90 & - & 0.3900 & 121.7509995 & - & 0.4200 & 8402 & yukF & BSU31920 & 0.8081850410 \\
\hline $200 \mathrm{nt}$ & 1467105 & 1467304 & forward & BSU13960 & ykwC & -407 & -41.17 & - & 0.3700 & 125.7789993 & - & 0.4650 & 602 & $\mathrm{pbpH}$ & BSU13980 & 0.8068280816 \\
\hline $200 \mathrm{nt}$ & 1406412 & 1406611 & forward & BSU13390 & ykoT & -1821 & -67.24 & - & 0.5600 & 121.6240005 & - & 0.3150 & 3402 & ykoX & BSU13430 & 0.8052443266 \\
\hline $200 \mathrm{nt}$ & 2897488 & 2897687 & forward & BSU28180 & ysxD & -17529 & -28.00 & - & 0.2150 & 125.7649994 & - & 0.4100 & 202 & ysnD & BSU28320 & 0.8036175966 \\
\hline $200 \mathrm{nt}$ & 1474672 & 1474871 & forward & BSU14010 & cheV & -57 & -50.60 & - & 0.3800 & 122.1959991 & - & 0.3650 & 3302 & ykuF & BSU14060 & 0.8031342626 \\
\hline $200 \mathrm{nt}$ & 746375 & 746574 & forward & BSU06780 & yeec & -277 & -31.50 & - & 0.2800 & 126.4580002 & - & 0.5000 & 3302 & yeeG & BSU06820 & 0.8004485369 \\
\hline
\end{tabular}

Significant hits of the forward and reverse strands (only showing forward strand here) of the B. subtilis intergenic regions having significantly high RND entropy (p-Val.< 0.0500 ) and LMFEGCRND probability higher than 0.8 . $100 \mathrm{nt}$ overlap used for $200 \mathrm{nt}$ scan (44847 segments). Distance from upstream and downstream operons are the distance from the center of the hit to the stop and start codons of upstream and downstream operons,

respectively. Probability denotes the likelihood of being a riboswitch under the LMFEGCRND model. Negative values indicate distance to upstream operon. Columns Ups seam/Downst ream Operon show gene ID within the operon. 
Table 26 Top entropy hits in B. subtilis reverse strand

\begin{tabular}{|c|c|c|c|c|c|c|c|c|c|c|c|c|c|c|c|}
\hline B. subtilis & Start & End & Strand Upstream operon & $\begin{array}{l}\text { Upstream } \\
\text { gene }\end{array}$ & $\begin{array}{l}\text { Dist. to } \\
\text { upstream }\end{array}$ & MFE & MFE p. Val. G & GC & RND & RND p. Val. & Uracil & $\begin{array}{l}\text { Dist. to } \\
\text { downstream }\end{array}$ & $\begin{array}{l}\text { Downstream } \\
\text { gene }\end{array}$ & $\begin{array}{l}\text { Downstream } \\
\text { operon }\end{array}$ & Probability \\
\hline $200 \mathrm{nt}$ & 3717398 & 3717597 & reverse BSU36100 & ywrD & -1637 & -51.30 & - & 0.3650 & 130.8540039 & - & 0.3950 & 399 & $\mathrm{cotH}$ & BSU36060 & 0.9702541828 \\
\hline $200 \mathrm{nt}$ & 3717498 & 3717697 & reverse BSU36100 & ywrD & -1537 & -50.60 & - & 0.3500 & 129.2720032 & - & 0.4000 & 499 & $\mathrm{cotH}$ & BSU36060 & 0.9603169560 \\
\hline $200 \mathrm{nt}$ & 4066209 & 4066408 & reverse BSU39600 & yxeC & -299 & -67.50 & - & 0.4900 & 125.4860001 & - & 0.3650 & 799 & yxeF & BSU39570 & 0.9434255362 \\
\hline $200 \mathrm{nt}$ & 786306 & 786505 & reverse BSU07220 & yetL & -3247 & -72.30 & - & 0.5600 & 125.9049988 & - & 0.3050 & 499 & yetH & BSU07160 & 0.9432973266 \\
\hline $200 \mathrm{nt}$ & 2249144 & 2249343 & reverse BSU21440 & $b d b B$ & -15982 & -49.00 & - & 0.3700 & 127.3170013 & - & 0.3850 & 1699 & youB & BSU21329 & 0.9216341376 \\
\hline $200 \mathrm{nt}$ & 2596201 & 2596400 & reverse BSU25170 & yqfo & -1202 & -42.96 & - & 0.3650 & 129.5290070 & - & 0.4950 & 799 & $\operatorname{csh} B$ & BSU25140 & 0.9206426144 \\
\hline $200 \mathrm{nt}$ & 3717298 & 3717497 & reverse BSU36100 & ywrD & -1737 & -45.43 & - & 0.3750 & 128.8240051 & - & 0.4200 & 299 & $\cot H$ & BSU36060 & 0.9199228883 \\
\hline $200 \mathrm{nt}$ & 3671576 & 3671775 & reverse BSU35700 & $\operatorname{tagH}$ & -1889 & -33.00 & - & 0.2350 & 128.6049957 & - & 0.4550 & 299 & ggaA & BSU35690 & 0.9113640189 \\
\hline $200 \mathrm{nt}$ & 3717598 & 3717797 & reverse BSU36100 & $y w r D$ & -1437 & -50.50 & - & 0.3850 & 126.3860016 & - & 0.4350 & 599 & $\cot H$ & BSU36060 & 0.9073441625 \\
\hline $200 \mathrm{nt}$ & 3373949 & 3374148 & reverse BSU32890 & yusQ & -2569 & -67.60 & - & 0.4800 & 122.0930023 & - & 0.3600 & 399 & fadM & BSU32850 & 0.8957566023 \\
\hline $200 \mathrm{nt}$ & 3666584 & 3666783 & reverse BSU35680 & ggaB & -526 & -44.06 & - & 0.3300 & 126.5329971 & - & 0.4650 & 1299 & mnaA & BSU35660 & 0.8957416415 \\
\hline $200 \mathrm{nt}$ & 3941142 & 3941341 & reverse BSU38430 & gspA & -3319 & -43.33 & - & 0.3950 & 128.7100067 & - & 0.4800 & 1599 & ywbA & BSU38390 & 0.8889677525 \\
\hline $200 \mathrm{nt}$ & 2879134 & 2879333 & reverse BSU28190 & engB & -649 & -38.80 & - & 0.2800 & 126.4779968 & - & 0.4100 & 99 & hemA & BSU28170 & 0.8852627277 \\
\hline $200 \mathrm{nt}$ & 3907615 & 3907814 & reverse BSU38100 & $y w c H$ & -2588 & -46.12 & - & 0.2950 & 123.9560013 & - & 0.3600 & 399 & ywcl & BSU38080 & 0.8837128878 \\
\hline $200 \mathrm{nt}$ & 1248822 & 1249021 & reverse BSU11740 & $\cot Z$ & -521 & -54.90 & - & 0.5300 & 128.1889954 & - & 0.4000 & 8799 & yjbP & BSU11630 & 0.8796436787 \\
\hline $200 \mathrm{nt}$ & 2004047 & 2004246 & reverse BSU18400 & yoeD & -116 & -40.10 & - & 0.3150 & 126.8629990 & - & 0.3900 & 199 & yoec & BSU18390 & 0.8789740205 \\
\hline $200 \mathrm{nt}$ & 3671676 & 3671875 & reverse BSU35700 & $\mathrm{tagH}$ & -1789 & -35.80 & - & 0.2600 & 126.5380020 & - & 0.3900 & 399 & ggaA & BSU35690 & 0.8741892576 \\
\hline $200 \mathrm{nt}$ & 2257744 & 2257943 & reverse BSU21440 & $b d b B$ & -7382 & -38.35 & - & 0.3350 & 128.0359955 & - & 0.4600 & 10299 & youB & BSU21329 & 0.8739098310 \\
\hline $200 \mathrm{nt}$ & 2294238 & 2294437 & reverse BSU21800 & ypkP & -1645 & -57.60 & - & 0.3900 & 122.0520020 & - & 0.3950 & 299 & ilvA & BSU21770 & 0.8724481463 \\
\hline $200 \mathrm{nt}$ & 791156 & 791355 & reverse BSU07240 & yetN & -207 & -70.84 & - & 0.5850 & 123.2480011 & - & 0.2900 & 1099 & yetL & BSU07220 & 0.8711011410 \\
\hline $200 \mathrm{nt}$ & 494742 & 494941 & reverse BSU04430 & $y d b D$ & -899 & -52.90 & - & 0.4250 & 125.0230026 & - & 0.4350 & 1399 & ydaT & BSU04380 & 0.8695754409 \\
\hline $200 \mathrm{nt}$ & 1355092 & 1355291 & reverse BSU12900 & htrA & -2745 & -71.91 & - & 0.5400 & 121.2139969 & - & 0.3050 & 2499 & $y \mathrm{kbA}$ & BSU12860 & 0.8692007065 \\
\hline $200 \mathrm{nt}$ & 737924 & 738123 & reverse BSU06740 & yefB & -972 & -70.30 & - & 0.5400 & 121.8629990 & - & 0.3150 & 5199 & yerO & BSU06700 & 0.8691427112 \\
\hline $200 \mathrm{nt}$ & 937998 & 938197 & reverse BSU08700 & ygaE & -3071 & -54.20 & - & 0.4350 & 124.7170029 & - & 0.4200 & 1099 & yfhs & BSU08640 & 0.8665797114 \\
\hline $200 \mathrm{nt}$ & 3421066 & 3421265 & reverse BSU33340 & sspJ & -300 & -64.50 & - & 0.4850 & 122.1780014 & - & 0.3150 & 99 & lysP & BSU33330 & 0.8648849726 \\
\hline $200 \mathrm{nt}$ & 2739937 & 2740136 & reverse BSU26830 & yrpE & -1321 & -49.69 & - & 0.4200 & 125.9660034 & - & 0.3800 & 3499 & aadk & BSU26790 & 0.8648592830 \\
\hline
\end{tabular}


Table 26 Top entropy hits in B. subtilis reverse strand (Continued)

\begin{tabular}{|c|c|c|c|c|c|c|c|c|c|c|c|c|c|c|c|c|}
\hline $200 \mathrm{nt}$ & 1097850 & 1098049 & reverse & BSU10230 & yhfH & -171 & -36.90 & - & 0.2550 & 125.4639969 & - & 0.4450 & 99 & gltT & BSU10220 & 0.8626419306 \\
\hline $200 \mathrm{nt}$ & 3851617 & 3851816 & reverse & BSU37520 & ywhD & -470 & -68.42 & - & 0.5500 & 122.7009964 & - & 0.3000 & 2099 & speE & BSU37500 & 0.8624630570 \\
\hline $200 \mathrm{nt}$ & 2724828 & 2725027 & reverse & BSU26660 & $\operatorname{yrdN}$ & -187 & -37.44 & - & 0.2200 & 124.0479965 & - & 0.3800 & 99 & $\mathrm{czcD}$ & BSU26650 & 0.8623370528 \\
\hline $200 \mathrm{nt}$ & 1204503 & 1204702 & reverse & BSU11270 & yjzD & -129 & -59.84 & - & 0.5050 & 124.6380005 & - & 0.3600 & 13299 & yitU & BSU11140 & 0.8622197509 \\
\hline $200 \mathrm{nt}$ & 2692345 & 2692544 & reverse & BSU26240 & yqaO & -201 & -47.40 & - & 0.3350 & 123.8980026 & - & 0.4100 & 999 & yqaQ & BSU26220 & 0.8618891239 \\
\hline $200 \mathrm{nt}$ & 3108926 & 3109125 & reverse & BSU30370 & bceB & -372 & -32.52 & - & 0.3250 & 129.4949951 & - & 0.5250 & 599 & $y t t B$ & BSU30350 & 0.8596788645 \\
\hline $200 \mathrm{nt}$ & 1493525 & 1493724 & reverse & BSU14250 & yknT & -779 & -39.60 & - & 0.2150 & 122.8750000 & - & 0.4750 & 1599 & ykuT & BSU14210 & 0.8589127660 \\
\hline $200 \mathrm{nt}$ & 2111609 & 2111808 & reverse & BSU19380 & yojO & -129 & -37.20 & - & 0.2700 & 125.5370026 & - & 0.4050 & 99 & sucA & BSU19370 & 0.8542534709 \\
\hline $200 \mathrm{nt}$ & 1678852 & 1679051 & reverse & BSU17060 & $y m z D$ & -101667 & -62.81 & - & 0.4750 & 122.0299988 & - & 0.3300 & 7299 & ylqB & BSU15960 & 0.8517054319 \\
\hline $200 \mathrm{nt}$ & 4109617 & 4109816 & reverse & BSU40030 & yхaB & -1233 & -52.14 & - & 0.3400 & 121.7229996 & - & 0.3900 & 99 & $y x a D$ & BSU40010 & 0.8503260016 \\
\hline $200 \mathrm{nt}$ & 3373849 & 3374048 & reverse & BSU32890 & yusQ & -2669 & -56.90 & - & 0.5000 & 125.1539993 & - & 0.3500 & 299 & fadM & BSU32850 & 0.8485122919 \\
\hline $200 \mathrm{nt}$ & 1886780 & 1886979 & reverse & BSU17590 & xylR & -3633 & -42.30 & - & 0.3150 & 124.7409973 & - & 0.3500 & 13299 & cwlC & BSU17410 & 0.8470579386 \\
\hline $200 \mathrm{nt}$ & 3153718 & 3153917 & reverse & BSU30850 & $y t d A$ & -918 & -51.80 & - & 0.3600 & 122.3779984 & - & 0.3500 & 99 & menF & BSU30830 & 0.8457853198 \\
\hline $200 \mathrm{nt}$ & 984466 & 984665 & reverse & BSU09120 & yhck & -1169 & -40.11 & - & 0.2800 & 124.3939972 & - & 0.3750 & 99 & $\operatorname{cspB}$ & BSU09100 & 0.8457109332 \\
\hline $200 \mathrm{nt}$ & 3464551 & 3464750 & reverse & BSU33780 & sdpl & -1784 & -40.60 & - & 0.3100 & 125.1740036 & - & 0.4450 & 1399 & opuBA & BSU33730 & 0.8446103930 \\
\hline $200 \mathrm{nt}$ & 3684271 & 3684470 & reverse & BSU35780 & lytD & -456 & -43.90 & - & 0.3450 & 125.0149994 & - & 0.3500 & 3399 & $\operatorname{tag} D$ & BSU35740 & 0.8443253636 \\
\hline $200 \mathrm{nt}$ & 2770775 & 2770974 & reverse & BSU27160 & сурB & -3016 & -41.20 & - & 0.3550 & 126.4410019 & - & 0.4050 & 2199 & yrhP & BSU27100 & 0.8441781402 \\
\hline $200 \mathrm{nt}$ & 1666288 & 1666487 & reverse & BSU15960 & ylqB & -4779 & -56.60 & - & 0.4500 & 123.3949966 & - & 0.3650 & 10599 & rpmB & BSU15820 & 0.8431691527 \\
\hline $200 \mathrm{nt}$ & 2343862 & 2344061 & reverse & BSU22330 & ypoC & -303 & -43.50 & - & 0.3800 & 126.2779999 & - & 0.4850 & 3199 & yppC & BSU22300 & 0.8418609500 \\
\hline $200 \mathrm{nt}$ & 3158854 & 3159053 & reverse & BSU30890 & $y t x O$ & -305 & -56.71 & - & 0.4200 & 122.1419983 & - & 0.3650 & 3399 & $y t d A$ & BSU30850 & 0.8375009298 \\
\hline $200 \mathrm{nt}$ & 4129648 & 4129847 & reverse & BSU40200 & yydD & -831 & -66.70 & - & 0.5050 & 120.9280014 & - & 0.3350 & 2099 & yydF & BSU40180 & 0.8359215260 \\
\hline $200 \mathrm{nt}$ & 4134175 & 4134374 & reverse & BSU40230 & yydA & -162 & -30.70 & - & 0.2450 & 126.6350021 & - & 0.3400 & 99 & yydB & BSU 40220 & 0.8344783783 \\
\hline $200 \mathrm{nt}$ & 245362 & 245561 & reverse & BSU02340 & gltP & -8057 & -57.10 & - & 0.4500 & 122.8600006 & - & 0.3500 & 1799 & ybfl & BSU02220 & 0.8332447410 \\
\hline $200 \mathrm{nt}$ & 3996389 & 3996588 & reverse & BSU38970 & $y x j F$ & -4051 & -40.50 & - & 0.3750 & 126.9710007 & - & 0.4250 & 4899 & yxkA & BSU38870 & 0.8313001394 \\
\hline $200 \mathrm{nt}$ & 2739837 & 2740036 & reverse & BSU26830 & yrpE & -1421 & -64.40 & - & 0.4950 & 121.3069992 & - & 0.3300 & 3399 & aadK & BSU26790 & 0.8294041157 \\
\hline $200 \mathrm{nt}$ & 1903178 & 1903377 & reverse & BSU17690 & yncM & -234 & -35.20 & - & 0.2700 & 125.4830017 & - & 0.4250 & 1899 & $\cot U$ & BSU17670 & 0.8289884329 \\
\hline $200 \mathrm{nt}$ & 933665 & 933864 & reverse & BSU08620 & yfhP & -693 & -62.04 & - & 0.4700 & 121.3809967 & - & 0.3750 & 5499 & sspK & BSU08550 & 0.8283531070 \\
\hline $200 \mathrm{nt}$ & 2054430 & 2054629 & reverse & BSU18840 & xynA & -70 & -52.10 & - & 0.3850 & 122.5159988 & - & 0.3450 & 599 & pps & BSU18830 & 0.8281581998 \\
\hline
\end{tabular}




\section{Table 26 Top entropy hits in B. subtilis reverse strand (Continued)}

\begin{tabular}{|c|c|c|c|c|c|c|c|c|c|c|c|c|c|c|c|}
\hline $200 \mathrm{nt}$ & 737824 & 738023 & reverse & BSU06740 & yefB & -1072 & -69.00 & - & 0.5350 & 120.7480011 & 0.3100 & 5099 & yerO & BSU06700 & 0.8277365565 \\
\hline $200 \mathrm{nt}$ & 198226 & 198425 & reverse & BSU01800 & alkA & -4222 & -30.81 & - & 0.3750 & 130.7449951 & 0.5150 & 4299 & ybbk & BSU01720 & 0.8267450333 \\
\hline $200 \mathrm{nt}$ & 3604668 & 3604867 & reverse & BSU35100 & $y v \mid D$ & -1995 & -39.30 & - & 0.2750 & 123.9219971 & 0.3550 & 199 & yvmC & BSU35070 & 0.8267388940 \\
\hline $200 \mathrm{nt}$ & 3098465 & 3098664 & reverse & BSU30310 & $y t w F$ & -3637 & -48.30 & - & 0.3850 & 123.9609985 & 0.4100 & 1999 & ytaP & BSU30250 & 0.8252500296 \\
\hline $200 \mathrm{nt}$ & 419514 & 419713 & reverse & BSU03690 & $y c z F$ & -150 & -54.00 & - & 0.4400 & 123.4860001 & 0.3300 & 1899 & dtpT & BSU03670 & 0.8242135644 \\
\hline $200 \mathrm{nt}$ & 2221888 & 2222087 & reverse & BSU21080 & yonl & -6769 & -36.60 & - & 0.3550 & 127.6279984 & 0.3150 & 599 & yonR & BSU21020 & 0.8236665726 \\
\hline $200 \mathrm{nt}$ & 2434023 & 2434222 & reverse & BSU23340 & урuв & -21 & -56.65 & - & 0.4750 & 123.5520020 & 0.3050 & 599 & ypz」 & BSU23328 & 0.8226841688 \\
\hline $200 \mathrm{nt}$ & 3241980 & 3242179 & reverse & BSU31590 & yufs & -4073 & -57.60 & - & 0.4600 & 122.6460037 & 0.3500 & 5099 & yufk & BSU31510 & 0.8222519755 \\
\hline $200 \mathrm{nt}$ & 1700752 & 1700951 & reverse & BSU17060 & $y m z D$ & -79767 & -52.70 & - & 0.4300 & 123.5100021 & 0.3150 & 29199 & ylqB & BSU15960 & 0.8189544678 \\
\hline $200 \mathrm{nt}$ & 2709820 & 2710019 & reverse & BSU26490 & yrkJ & -83 & -48.40 & - & 0.3600 & 122.8440018 & 0.3550 & 499 & yrkK & BSU26480 & 0.8178487420 \\
\hline $200 \mathrm{nt}$ & 153939 & 154138 & reverse & BSU01550 & gerD & -4477 & -50.70 & - & 0.4100 & 123.6019974 & 0.3700 & 108899 & abrB & BSU00370 & 0.8176639676 \\
\hline $200 \mathrm{nt}$ & 3239080 & 3239279 & reverse & BSU31590 & yufs & -6973 & -58.30 & - & 0.4150 & 120.6800003 & 0.3750 & 2199 & yufk & BSU31510 & 0.8171101809 \\
\hline $200 \mathrm{nt}$ & 3467327 & 3467526 & reverse & BSU33800 & opuCD & -120 & -32.10 & - & 0.2350 & 125.1719971 & 0.3550 & 99 & $\operatorname{sdpR}$ & BSU33790 & 0.8168275952 \\
\hline $200 \mathrm{nt}$ & 543114 & 543313 & reverse & BSU05000 & yddK & -2953 & -43.99 & - & 0.3600 & 124.5599976 & 0.4700 & 11699 & $\mathrm{immR}$ & BSU04820 & 0.8156080246 \\
\hline $200 \mathrm{nt}$ & 3108826 & 3109025 & reverse & BSU30370 & bceB & -472 & -37.52 & - & 0.3400 & 126.4919968 & 0.4900 & 499 & yttB & BSU30350 & 0.8153505921 \\
\hline $200 \mathrm{nt}$ & 3334388 & 3334587 & reverse & BSU32470 & pucE & -1264 & -54.50 & - & 0.4850 & 124.4670029 & 0.3050 & 5899 & pucH & BSU32410 & 0.8130649924 \\
\hline $200 \mathrm{nt}$ & 3684371 & 3684570 & reverse & BSU35780 & lytD & -356 & -42.00 & - & 0.3250 & 124.1009979 & 0.3650 & 3499 & $\operatorname{tag} \mathrm{D}$ & BSU35740 & 0.8130072355 \\
\hline $200 \mathrm{nt}$ & 881307 & 881506 & reverse & BSU08120 & yfjF & -4438 & -52.36 & - & 0.4850 & 125.3059998 & 0.3900 & 9099 & yfjQ & BSU08000 & 0.8121696115 \\
\hline $200 \mathrm{nt}$ & 2926798 & 2926997 & reverse & BSU28630 & pheT & -111 & -60.46 & - & 0.4950 & 122.2929993 & 0.3200 & 999 & yshA & BSU28610 & 0.8096395731 \\
\hline $200 \mathrm{nt}$ & 4066309 & 4066508 & reverse & BSU39600 & yxeC & -199 & -55.60 & - & 0.4600 & 123.0559998 & 0.3600 & 899 & yxeF & BSU39570 & 0.8090547919 \\
\hline $200 \mathrm{nt}$ & 3688648 & 3688847 & reverse & BSU35830 & ywtG & -3786 & -49.93 & - & 0.3300 & 120.9260025 & 0.3550 & 199 & yvyl & BSU35790 & 0.8084035516 \\
\hline $200 \mathrm{nt}$ & 1668788 & 1668987 & reverse & BSU15960 & ylaB & -2279 & -51.60 & - & 0.4050 & 122.7809982 & 0.4150 & 13099 & rpmB & BSU15820 & 0.8080439568 \\
\hline $200 \mathrm{nt}$ & 3334288 & 3334487 & reverse & BSU32470 & pucE & -1364 & -62.06 & - & 0.4700 & 120.7320023 & 0.2950 & 5799 & pucH & BSU32410 & 0.8073683381 \\
\hline $200 \mathrm{nt}$ & 3723732 & 3723931 & reverse & BSU36170 & ywqL & -589 & -55.50 & - & 0.4550 & 122.8669968 & 0.3300 & 499 & $y w q N$ & BSU36150 & 0.8070057034 \\
\hline $200 \mathrm{nt}$ & 899787 & 899986 & reverse & BSU08340 & padR & -9312 & -54.60 & - & 0.3900 & 121.0039978 & 0.3550 & 10199 & yfjA & BSU08170 & 0.8061554432 \\
\hline $200 \mathrm{nt}$ & 2813434 & 2813633 & reverse & BSU27540 & yrvM & -110 & -48.20 & - & 0.3850 & 123.3710022 & 0.4200 & 1399 & cymR & BSU27520 & 0.8043378592 \\
\hline $200 \mathrm{nt}$ & 3458216 & 3458415 & reverse & BSU33700 & opuBD & -1491 & -44.09 & - & 0.3500 & 123.8420029 & 0.4200 & 1799 & $y v z C$ & BSU33650 & 0.8041363358 \\
\hline $200 \mathrm{nt}$ & 3918262 & 3918461 & reverse & BSU38190 & galT & -732 & -37.50 & - & 0.2900 & 124.4739990 & 0.3100 & 99 & qoxA & BSU38170 & 0.8040998578 \\
\hline
\end{tabular}


Table 26 Top entropy hits in B. subtilis reverse strand (Continued)

\begin{tabular}{|c|c|c|c|c|c|c|c|c|c|c|c|c|c|c|c|}
\hline $200 \mathrm{nt}$ & 2576788 & 2576987 & reverse & BSU24950 & pstBB & -323 & -53.30 & - & 0.3700 & 120.7910004 & 0.4100 & 699 & yqgL & BSU24920 & 0.8040402532 \\
\hline $200 \mathrm{nt}$ & 2316211 & 2316410 & reverse & BSU22040 & ypbQ & -136 & -50.40 & - & 0.3350 & 120.7730026 & 0.3850 & 199 & ypbR & BSU22030 & 0.8038558364 \\
\hline $200 \mathrm{nt}$ & 2249344 & 2249543 & reverse & BSU21440 & $\mathrm{bdbB}$ & -15782 & -43.38 & - & 0.3500 & 124.1179962 & 0.4650 & 1899 & youB & BSU21329 & 0.8037469983 \\
\hline $200 \mathrm{nt}$ & 2333112 & 2333311 & reverse & BSU22210 & yprB & -113 & -43.21 & - & 0.3300 & 123.4840012 & 0.4200 & 199 & $\cot D$ & BSU22200 & 0.8028732538 \\
\hline $200 \mathrm{nt}$ & 2116670 & 2116869 & reverse & BSU19420 & yojk & -282 & -43.45 & - & 0.3300 & 123.3679962 & 0.3150 & 99 & cwls & BSU19410 & 0.8022140265 \\
\hline $200 \mathrm{nt}$ & 3941242 & 3941441 & reverse & BSU38430 & gspA & -3219 & -42.84 & - & 0.4300 & 126.9950027 & 0.4600 & 1699 & $y w b A$ & BSU38390 & 0.8019362092 \\
\hline $200 \mathrm{nt}$ & 1248722 & 1248921 & reverse & BSU11740 & $\cot Z$ & -621 & -64.39 & - & 0.5300 & 121.6650009 & 0.3550 & 8699 & yjbP & BSU11630 & 0.8019282818 \\
\hline $200 \mathrm{nt}$ & 3014345 & 3014544 & reverse & BSU29460 & moaB & -70 & -39.90 & - & 0.2550 & 122.2269974 & 0.3300 & 99 & $\operatorname{argG}$ & BSU29450 & 0.8009542227 \\
\hline $200 \mathrm{nt}$ & 2542225 & 2542424 & reverse & BSU24510 & yqho & -115 & -57.14 & - & 0.4800 & 122.8730011 & 0.4100 & 1499 & yqhR & BSU24480 & 0.8008475304 \\
\hline $200 \mathrm{nt}$ & 2096086 & 2096285 & reverse & BSU19230 & yoc」 & -165 & -39.50 & - & 0.2800 & 123.2190018 & 0.4150 & 399 & yocl & BSU19220 & 0.8003621101 \\
\hline $200 \mathrm{nt}$ & 4107928 & 4108127 & reverse & BSU40010 & $y x a D$ & -1158 & -51.01 & - & 0.4150 & 123.1330032 & 0.3800 & 99 & $y x a F$ & BSU39990 & 0.8002312183 \\
\hline $200 \mathrm{nt}$ & 2659771 & 2659970 & reverse & BSU25880 & yqxJ & -3681 & -49.60 & - & 0.3800 & 122.5149994 & 0.3600 & 1099 & yqcl & BSU25820 & 0.8001416922 \\
\hline
\end{tabular}

Significant hits of the forward and reverse strands (only showing reverse strand here) of the $B$. subtilis intergenic regions having significantly high RND entropy (p-Val.<0.0500) and LMFEGCRND probability higher than 0.8 . $100 \mathrm{nt}$ overlap used for $200 \mathrm{nt}$ scan (44847 segments). Distance from upstream and downstream operons are the distance from the center of the hit to the stop and start codons of upstream and downstream operons, respectively. Probability denotes the multinomial regression likelihood of being a riboswitch under the LMFEGCRND model. Negative values indicate distance to upstream operon. Columns Upsream/Downstream Operon show gene ID within the operon. 
Table 27 Top classification hits in E. coli

\begin{tabular}{|c|c|c|c|c|c|c|c|c|c|}
\hline R & Start & End & Strand & Upstream operon & Dist. to upstream & Uracil & Dist. to downstream & Downstream operon & Probability \\
\hline 1 & 384006 & 384105 & forward & ins $C-1$, ins CD-1, insD-1 & -2154 & 0.52 & 402 & tauA,tauB,tauC,tauD & 0.942 \\
\hline 2 & 237185 & 237284 & forward & aspV & -129 & 0.47 & 102 & yaft & 0.934 \\
\hline 3 & 2777119 & 2777218 & forward & yfjX,yfjY,yfjZ,ypjF,ypj」 & -1266 & 0.38 & 7252 & ygaQ_1,ygaQ_2 & 0.925 \\
\hline 4 & 2304856 & 2304955 & forward & eco & -2392 & 0.45 & 6202 & micF & 0.923 \\
\hline 5 & 83968 & 84067 & forward & setA,sgrS,sgrT & -5120 & 0.49 & 352 & leuO & 0.92 \\
\hline 6 & 2902496 & 2902595 & reverse & queE & -224 & 0.48 & 4249 & ygcw & 0.918 \\
\hline 7 & 294815 & 294914 & forward & yag」 & -3311 & 0.43 & 7352 & yagU & 0.914 \\
\hline 8 & 4554566 & 4554665 & forward & uxuR & -1145 & 0.48 & 402 & iraD & 0.913 \\
\hline 9 & 405479 & 405578 & forward & yail & 16 & 0.45 & 102 & aroL,aroM,yaiA & 0.908 \\
\hline 10 & 4570237 & 4570336 & forward & yjis & -250 & 0.38 & 152 & yjiT & 0.906 \\
\hline 11 & 754000 & 754099 & forward & nei,ybgl,ybgJ,ybgk,ybgL & -8002 & 0.4 & 352 & $\operatorname{sdh} A^{[1]}$ & 0.905 \\
\hline 12 & 2054653 & 2054752 & reverse & asnW & -1349 & 0.44 & 3349 & yeeL_1,yeeL_2 & 0.905 \\
\hline 13 & 2202241 & 2202340 & reverse & yehs & -7458 & 0.44 & 10099 & mrp & 0.903 \\
\hline 14 & 330995 & 331094 & forward & betT & -226 & 0.52 & 552 & yahA & 0.9 \\
\hline 15 & 3183291 & 3183390 & reverse & glgs & -6421 & 0.44 & 599 & ribB,sroG & 0.9 \\
\hline 16 & 384056 & 384155 & forward & ins $C-1$, ins CD-1, insD- 1 & -2204 & 0.43 & 352 & tauA,tauB,tauC,tauD & 0.898 \\
\hline 17 & 4570187 & 4570286 & forward & yjis & -200 & 0.42 & 202 & yjiT & 0.898 \\
\hline 18 & 557285 & 557384 & forward & cysS & -2017 & 0.35 & 102 & sfmA & 0.894 \\
\hline 19 & 3190062 & 3190161 & reverse & sibD & -2632 & 0.37 & 149 & glgs & 0.893 \\
\hline 20 & 1543575 & 1543674 & forward & nhoA & -10633 & 0.45 & 1802 & $\mathrm{fdnG}, \mathrm{fdnH}, \mathrm{fdnl}$ & 0.89 \\
\hline 21 & 2190295 & 2190394 & reverse & yehE & -193 & 0.46 & 99 & yehA,yehB,yehC,yehD & 0.89 \\
\hline 22 & 3181507 & 3181606 & reverse & ribB,sroG & -279 & 0.43 & 1099 & ygiD & 0.89 \\
\hline 23 & 3834703 & 3834802 & reverse & nlpA & -2446 & 0.37 & 799 & yicl,yicJ & 0.889 \\
\hline 24 & 1753166 & 1753265 & reverse & ynhG & -2530 & 0.45 & 49 & $y d h z$ & 0.888 \\
\hline 25 & 819916 & 820015 & forward & $y b h L$ & -56 & 0.41 & 52 & ybhM & 0.887 \\
\hline 26 & 2901746 & 2901845 & reverse & queE & -974 & 0.51 & 3499 & ygcw & 0.887 \\
\hline 27 & 651208 & 651307 & forward & $y b d R$ & -8610 & 0.49 & 202 & dpiA,dpiB & 0.886 \\
\hline
\end{tabular}


Table 27 Top classification hits in E. coli (Continued)

\begin{tabular}{|c|c|c|c|c|c|c|c|c|c|}
\hline 28 & 584973 & 585072 & forward & appY & -1271 & 0.57 & 9802 & cusA,cusB,cusC,cusF & 0.886 \\
\hline 29 & 2362398 & 2362497 & reverse & ais & -593 & 0.48 & 149 & yfaZ & 0.886 \\
\hline 30 & 1596214 & 1596313 & forward & osmC & -41085 & 0.39 & 3252 & IsrA,IsrB,IsrC,IsrD,IsrF,IsrG,tam & 0.882 \\
\hline 31 & 522335 & 522434 & forward & $y b b A, y b b P$ & -232 & 0.39 & 102 & rhsD,ybbC,ybbD,ylbH & 0.881 \\
\hline 32 & 3490420 & 3490519 & reverse & php,yhfS,yhfT,yhfU,yhfW,yhfx & -12488 & 0.39 & 149 & ppiA & 0.88 \\
\hline 33 & 1986023 & 1986122 & reverse & yecH & -1203 & 0.45 & 49 & isrB & 0.879 \\
\hline 34 & 3217299 & 3217398 & reverse & ygjH & -1589 & 0.42 & 249 & aer & 0.878 \\
\hline 35 & 2714626 & 2714725 & forward & eamB & -545 & 0.45 & 102 & ung & 0.877 \\
\hline 36 & 3984255 & 3984354 & forward & aslB & -1990 & 0.42 & 152 & glmZ & 0.877 \\
\hline 37 & 2651611 & 2651710 & reverse & sseB & -519 & 0.5 & 99 & C0614 & 0.877 \\
\hline 38 & 4516300 & 4516399 & forward & insO-2,yjhV,yjhW & -8095 & 0.39 & 202 & ins $A-7$ & 0.876 \\
\hline 39 & 3886253 & 3886352 & reverse & purP & -6993 & 0.31 & 4549 & dnaA,dnaN,recF & 0.876 \\
\hline 40 & 1577414 & 1577513 & forward & osmC & -22285 & 0.41 & 22052 & IsrA,IsrB,IsrC,IsrD,IsrF,IsrG,tam & 0.875 \\
\hline 41 & 776349 & 776448 & reverse & zitB & -6707 & 0.39 & 11299 & mngR & 0.874 \\
\hline 42 & 1543625 & 1543724 & forward & nhoA & -10683 & 0.46 & 1752 & $\mathrm{fdnG}, \mathrm{fdnH}, \mathrm{fdnl}$ & 0.871 \\
\hline 43 & 29201 & 29300 & forward & dapB & 43 & 0.49 & 402 & $\operatorname{car} A, \operatorname{carB}$ & 0.871 \\
\hline 44 & 1542975 & 1543074 & forward & nhoA & -10033 & 0.39 & 2402 & $\mathrm{fdnG}, \mathrm{fdnH}, \mathrm{fdnl}$ & 0.871 \\
\hline 45 & 3768179 & 3768278 & reverse & yibH,yibl & -38 & 0.41 & 8249 & yibF & 0.871 \\
\hline 46 & 3631114 & 3631213 & forward & yhhl & -7528 & 0.4 & 1702 & yhiM & 0.87 \\
\hline 47 & 2166486 & 2166585 & forward & cyaR & -1213 & 0.41 & 202 & yegs & 0.87 \\
\hline 48 & 4578972 & 4579071 & forward & symR & -989 & 0.38 & 5952 & mrr & 0.869 \\
\hline 49 & 522235 & 522334 & forward & $y b b A, y b b P$ & -132 & 0.39 & 202 & rhsD,ybbC,ybbD,ylbH & 0.869 \\
\hline 50 & 2383795 & 2383894 & reverse & $y f b N$ & -1888 & 0.48 & 99 & yfbK & 0.869 \\
\hline 51 & 4577258 & 4577357 & forward & yjiV & -2331 & 0.36 & 552 & symR & 0.868 \\
\hline 52 & 153855 & 153954 & forward & yadD & -5936 & 0.37 & 8202 & hrpB & 0.868 \\
\hline 53 & 3665704 & 3665803 & reverse & yhjA & -61 & 0.42 & 149 & $\operatorname{gadA}, \operatorname{gadW}, \operatorname{gadX}$ & 0.868 \\
\hline 54 & 3651672 & 3651771 & reverse & hdeA,hdeB,yhiD & -1557 & 0.45 & 499 & ins $\mathrm{H}-11$ & 0.868 \\
\hline 55 & 4619642 & 4619741 & forward & deoA,deoB,deoC,deoD & 32 & 0.38 & 102 & yjj」 & 0.867 \\
\hline
\end{tabular}


Table 27 Top classification hits in E. coli (Continued)

\begin{tabular}{|c|c|c|c|c|c|c|c|c|c|}
\hline 56 & 1645875 & 1645974 & reverse & ynfP & -4828 & 0.45 & 49 & dicC,ydfW,ydfx & 0.867 \\
\hline 57 & 4554516 & 4554615 & forward & uxuR & -1095 & 0.42 & 452 & iraD & 0.866 \\
\hline 58 & 4539860 & 4539959 & forward & fimB & -229 & 0.41 & 152 & fimE & 0.866 \\
\hline 59 & 1588711 & 1588810 & reverse & hipA,hipB & -118 & 0.4 & 199 & ynel & 0.866 \\
\hline 60 & 3181557 & 3181656 & reverse & ribB,sroG & -229 & 0.33 & 1149 & ygiD & 0.865 \\
\hline 61 & 269657 & 269756 & reverse & ins $\mathrm{H}-1$ & -3619 & 0.45 & 299 & perR & 0.864 \\
\hline 62 & 3925028 & 3925127 & forward & $\mathrm{cbrB}, \mathrm{cbrC}$ & -28347 & 0.47 & 102 & asnA & 0.863 \\
\hline 63 & 4538580 & 4538679 & forward & yjhR & -4477 & 0.43 & 352 & fimB & 0.863 \\
\hline 64 & 4077095 & 4077194 & reverse & fdhE,fdoG,fdoH,fdol & -1178 & 0.39 & 5549 & yihS,yihT,yihU & 0.863 \\
\hline 65 & 1210379 & 1210478 & reverse & iraM & -475 & 0.42 & 1549 & stfE,tfaE & 0.862 \\
\hline 66 & 4213351 & 4213450 & forward & metA & -70 & 0.37 & 102 & aceA,aceB,acek & 0.861 \\
\hline 67 & 578853 & 578952 & forward & essD,rrrD,rzpD & -1013 & 0.49 & 202 & $\mathrm{ybcW}$ & 0.861 \\
\hline 68 & 3755951 & 3756050 & reverse & selA,selB & -40 & 0.33 & 149 & yiaY & 0.861 \\
\hline 69 & 924768 & 924867 & forward & $\mathrm{clpA}$ & 44 & 0.36 & 7002 & $\operatorname{lrp}$ & 0.86 \\
\hline 70 & 2520650 & 2520749 & reverse & харА,харВ & -52 & 0.42 & 199 & xapR & 0.86 \\
\hline 71 & 582454 & 582553 & forward & $\operatorname{tfax}$ & -122 & 0.44 & 402 & appY & 0.859 \\
\hline 72 & 1049984 & 1050083 & forward & ins $A-4$,ins $A B-4$, ins $B-4$ & -182 & 0.38 & 652 & cspG & 0.859 \\
\hline 73 & 3767704 & 3767803 & forward & yibG & -994 & 0.36 & 2552 & $m t|A, m t| D, m t \mid R$ & 0.859 \\
\hline 74 & 2383745 & 2383844 & reverse & yfbN & -1938 & 0.45 & 49 & yfbK & 0.859 \\
\hline 75 & 1005025 & 1005124 & forward & pyrD & 25 & 0.46 & 102 & zapC & 0.858 \\
\hline 76 & 157105 & 157204 & forward & yadD & -9186 & 0.34 & 4952 & hrpB & 0.858 \\
\hline 77 & 1669567 & 1669666 & reverse & mdtl,mdt」 & -1228 & 0.46 & 1999 & ynfL & 0.858 \\
\hline 78 & 3800263 & 3800362 & forward & rfaD,waaC,waaF,waaL & -3984 & 0.35 & 6252 & coaD,waaA & 0.856 \\
\hline 79 & 2784960 & 2785059 & reverse & ygaU & -9350 & 0.41 & 1149 & ileY & 0.856 \\
\hline 80 & 2468130 & 2468229 & reverse & yfdK,yfdL,yfdM,yfdN,yfdO & -920 & 0.49 & 5149 & mlaA & 0.855 \\
\hline 81 & 2991883 & 2991982 & reverse & ygeK,ygeL & -550 & 0.39 & 3549 & yqeK & 0.855 \\
\hline 82 & 2859337 & 2859436 & reverse & nlpD,rpos & -5195 & 0.42 & 99 & ygbl & 0.855 \\
\hline 83 & 3490370 & 3490469 & reverse & php,yhfs,yhfT,yhfU,yhfW,yhfx & -12538 & 0.32 & 99 & ppiA & 0.854 \\
\hline
\end{tabular}


Table 27 Top classification hits in E. coli (Continued)

\begin{tabular}{|c|c|c|c|c|c|c|c|c|c|}
\hline 84 & 4220501 & 4220600 & forward & aceA,aceB,aceK & -2097 & 0.37 & 1302 & metH & 0.853 \\
\hline 85 & 715871 & 715970 & reverse & potE,speF & -249 & 0.37 & 99 & ybfG,ybfH & 0.853 \\
\hline 86 & 2461957 & 2462056 & reverse & mlaA & -268 & 0.45 & 3049 & $y f c Z$ & 0.852 \\
\hline 87 & 2876502 & 2876601 & reverse & cas1,cas2, casA,casB,casC, casD, casE & -40 & 0.34 & 2199 & cysC,cysD, cysN & 0.851 \\
\hline 88 & 4084875 & 4084974 & forward & fdhD & 46 & 0.42 & 102 & yiiG & 0.85 \\
\hline 89 & 4535630 & 4535729 & forward & yjhR & -1527 & 0.31 & 3302 & fimB & 0.849 \\
\hline 90 & 1635392 & 1635491 & reverse & gnsB & -192 & 0.42 & 1049 & nohA,tfaQ,ydfN & 0.849 \\
\hline 91 & 3497220 & 3497319 & reverse & php,yhfS,yhfT,yhfU,yhfW,yhfx & -5688 & 0.33 & 6949 & ppiA & 0.849 \\
\hline 92 & 3266938 & 3267037 & reverse & gark,garL,garP,garR,rnpB & -1251 & 0.35 & 1899 & $\operatorname{tdc} A, \operatorname{tdcB}, \operatorname{tdc} c, t d c D, t d c E, t d c F, t d c G$ & 0.848 \\
\hline 93 & 2267568 & 2267667 & reverse & yejG & -8298 & 0.34 & 4299 & yeiW & 0.848 \\
\hline 94 & 3580990 & 3581089 & reverse & ggt & -2065 & 0.4 & 1999 & ryhB & 0.848 \\
\hline 95 & 1811219 & 1811318 & reverse & cedA & -177 & 0.5 & 99 & ydjO & 0.845 \\
\hline 96 & 583210 & 583309 & reverse & envY,ompT & -644 & 0.4 & 1299 & $y b c Y$ & 0.845 \\
\hline 97 & 3576850 & 3576949 & reverse & yhhW & -74 & 0.33 & 149 & gntK,gntR,gntU & 0.844 \\
\hline 98 & 3266488 & 3266587 & reverse & gark,garL,garP,garR,rnpB & -1701 & 0.43 & 1449 & $\operatorname{tdcA}, \operatorname{tdcB}, t d c C, t d c D, t d c E, t d c F, t d c G$ & 0.843 \\
\hline 99 & 2055603 & 2055702 & reverse & asnW & -399 & 0.33 & 4299 & yeeL_1,yeeL_2 & 0.843 \\
\hline 100 & 2739273 & 2739372 & reverse & rimM,rplS,rpsP,trmD & -2883 & 0.3 & 149 & aroF,tyrA & 0.842 \\
\hline
\end{tabular}

Top 50 hits of the forward and reverse strands of the $E$. coli intergenic regions using $50 \mathrm{nt}$-overlap $100 \mathrm{nt}$ window and under the LMFEGCRND model. The ranking of each hit is denoted in column R. Distance from upstream and downstream operons are the distance from the center of the hit to the stop and start codons of upstream and downstream operons, respectively. Probability denotes the multinomial regression likelihood of being a riboswitch under the LMFEGCRND model. Positions are according to gbU00096.2 version of E. coli and not gbU00096.3 version.

${ }^{1}$ Table 27: Complete list of genes in this operon is sdhA,sdhB,sdhC,sdhD, sucA, sucB,sucC,sucD. 


\section{Table 28 Top classification hits in E. coli uracil-comp. constrained}

\begin{tabular}{|c|c|c|c|c|c|c|c|c|c|}
\hline $\mathbf{R}$ & Start & End & Strand & Upstream operon & $\begin{array}{l}\text { Dist. to } \\
\text { upstream }\end{array}$ & Uracil & $\begin{array}{l}\text { Dist. to } \\
\text { downstream }\end{array}$ & $\begin{array}{l}\text { Downstream } \\
\text { operon }\end{array}$ & Probability \\
\hline 1 & 3886253 & 3886352 & reverse & purP & -6993 & 0.31 & 4549 & dnaA,dnaN,recF & 0.876 \\
\hline 2 & 3181557 & 3181656 & reverse & ribB,sroG & -229 & 0.33 & 1149 & ygiD & 0.865 \\
\hline 3 & 3755951 & 3756050 & reverse & selA,selB & -40 & 0.33 & 149 & yiaY & 0.861 \\
\hline 4 & 3490370 & 3490469 & reverse & php,yhfS,yhfT,yhfU,yhfW,yhfx & -12538 & 0.32 & 99 & ppiA & 0.854 \\
\hline 5 & 4535630 & 4535729 & forward & yjhR & -1527 & 0.31 & 3302 & $\mathrm{fimB}$ & 0.849 \\
\hline 6 & 3497220 & 3497319 & reverse & php,yhfS,yhfT,yhfU,yhfW,yhfx & -5688 & 0.33 & 6949 & ppiA & 0.849 \\
\hline 7 & 3576850 & 3576949 & reverse & yhhw & -74 & 0.33 & 149 & gntK,gntR,gntU & 0.844 \\
\hline 8 & 2055603 & 2055702 & reverse & asnW & -399 & 0.33 & 4299 & yeeL_1,yeeL_2 & 0.843 \\
\hline 9 & 2739273 & 2739372 & reverse & rimM,rplS,rpsP,trmD & -2883 & 0.3 & 149 & aroF,tyrA & 0.842 \\
\hline 10 & 2698570 & 2698669 & reverse & acpS,era,pdxJ,recO,rnc & -21 & 0.31 & 399 & shoB & 0.84 \\
\hline 11 & 3945101 & 3945200 & reverse & hdfR & -1 & 0.3 & 5799 & hsrA,yieP & 0.835 \\
\hline 12 & 2739223 & 2739322 & reverse & rimM,rpls,rpsP,trmD & -2933 & 0.31 & 99 & aroF,tyrA & 0.832 \\
\hline 13 & 3453521 & 3453620 & reverse & $\mathrm{bfd}, \mathrm{bfr}$ & -10701 & 0.32 & 149 & gspA,gspB & 0.825 \\
\hline 14 & 4274265 & 4274364 & reverse & soxs & -769 & 0.32 & 1249 & yjcB & 0.822 \\
\hline 15 & 1467282 & 1467381 & forward & ydbA_1 & -1259 & 0.32 & 52 & insl-2 & 0.82 \\
\hline 16 & 3116880 & 3116979 & forward & pheV & -8368 & 0.33 & 2452 & C0719 & 0.819 \\
\hline 17 & 790896 & 790995 & forward & aroG & -4939 & 0.26 & 3052 & acrZ & 0.817 \\
\hline 18 & 2777069 & 2777168 & forward & yfjX,yfjY,yfjZ,ypjF,ypjJ & -1216 & 0.33 & 7302 & ygaQ_1,ygaQ_2 & 0.817 \\
\hline 19 & 3189691 & 3189790 & reverse & glgs & -21 & 0.31 & 6999 & ribB,sroG & 0.817 \\
\hline 20 & 1694096 & 1694195 & reverse & uidR & -341 & 0.33 & 49 & uidA,uidB, uidC & 0.815 \\
\hline 21 & 2823699 & 2823798 & reverse & norR & -5049 & 0.31 & 149 & $\mathrm{mltB}$ & 0.81 \\
\hline 22 & 1805258 & 1805357 & reverse & yniB & -1414 & 0.32 & 1199 & ydiY & 0.809 \\
\hline 23 & 3382541 & 3382640 & reverse & yhcO & -1289 & 0.33 & 299 & mdh & 0.809 \\
\hline 24 & 1868697 & 1868796 & reverse & yoal & -3356 & 0.29 & 4249 & mipA & 0.808 \\
\hline 25 & 2386449 & 2386548 & reverse & nuo $A^{1}$ & -1572 & 0.31 & 49 & yfbN & 0.807 \\
\hline 26 & 3617057 & 3617156 & reverse & rbbA,yhhJ,yhil & -6596 & 0.3 & 7349 & yhhs & 0.806 \\
\hline
\end{tabular}




\section{Table 28 Top classification hits in E. coli uracil-comp. constrained (Continued)}

\begin{tabular}{|c|c|c|c|c|c|c|c|c|c|}
\hline 27 & 2815604 & 2815703 & forward & micA & -2660 & 0.29 & 8202 & gutM,gutQ,srlA,srlB,srlD,srlE,srlR & 0.805 \\
\hline 28 & 2943865 & 2943964 & reverse & mltA & -189 & 0.31 & 49 & $\operatorname{tcd} A$ & 0.804 \\
\hline 29 & 1983499 & 1983598 & forward & uspC & -5245 & 0.31 & 1402 & $\mathrm{ftnB}$ & 0.803 \\
\hline 30 & 150155 & 150254 & forward & yadD & -2236 & 0.32 & 11902 & hrpB & 0.801 \\
\hline 31 & 2553093 & 2553192 & forward & amiA,hemF & -898 & 0.33 & 3652 & intZ & 0.8 \\
\hline 32 & 2876452 & 2876551 & reverse & $\operatorname{cas} 1, \operatorname{cas} 2, \operatorname{cas} A, \operatorname{cas} B, \operatorname{cas} C, \operatorname{cas} D, \operatorname{cas} E$ & -90 & 0.31 & 2149 & cysC,cysD,cysN & 0.8 \\
\hline 33 & 1217949 & 1218048 & reverse & ymgD,ymgG & -3530 & 0.33 & 3299 & bluF & 0.8 \\
\hline 34 & 3346238 & 3346337 & reverse & elbB,mtgA & -816 & 0.33 & 8199 & $\mathrm{mlaB}, \mathrm{mlaC}, \mathrm{mlaD}, \mathrm{mlaE}, \mathrm{mlaF}$ & 0.798 \\
\hline 35 & 1174989 & 1175088 & reverse & $y c f Z, y m f A$ & -4664 & 0.3 & 649 & yсfT & 0.798 \\
\hline 36 & 4298873 & 4298972 & forward & gltP & -5007 & 0.26 & 12452 & rpiB & 0.797 \\
\hline 37 & 585173 & 585272 & forward & appY & -1471 & 0.29 & 9602 & cusA,cusB,cusC,cusF & 0.796 \\
\hline 38 & 1397665 & 1397764 & forward & yna & -1970 & 0.32 & 5052 & abgR & 0.795 \\
\hline 39 & 4492546 & 4492645 & forward & IptF,IptG & -6074 & 0.29 & 52 & idnK & 0.795 \\
\hline 40 & 655892 & 655991 & reverse & $\mathrm{crcB}$ & -837 & 0.32 & 749 & dcuC & 0.795 \\
\hline 41 & 266028 & 266127 & reverse & yafZ,ykfA & -331 & 0.28 & 299 & yafw² & 0.795 \\
\hline 42 & 2033559 & 2033658 & reverse & yedV,yedW & -1210 & 0.32 & 2099 & yedJ,yedR & 0.794 \\
\hline 43 & 3963904 & 3964003 & reverse & aslA & -18422 & 0.32 & 299 & rhlB & 0.794 \\
\hline 44 & 1463517 & 1463616 & reverse & insC-2,insCD-2,insD-2 & -2379 & 0.33 & 11899 & paaZ & 0.794 \\
\hline 45 & 2650116 & 2650215 & forward & xseA & -16443 & 0.31 & 352 & sseA & 0.793 \\
\hline 46 & 582654 & 582753 & forward & $\operatorname{tfax}$ & -322 & 0.3 & 202 & appY & 0.793 \\
\hline 47 & 4076645 & 4076744 & reverse & fdhE,fdoG,fdoH,fdol & -1628 & 0.29 & 5099 & yihs,yihT,yihU & 0.793 \\
\hline 48 & 2033409 & 2033508 & reverse & yedV,yedW & -1360 & 0.32 & 1949 & yedJ,yedR & 0.793 \\
\hline 49 & 1762598 & 1762697 & forward & Ipp & -6868 & 0.27 & 4452 & ydik & 0.792 \\
\hline 50 & 4501881 & 4501980 & forward & yjgZ & -2220 & 0.32 & 152 & yjhB,yjhC & 0.792 \\
\hline 51 & 1676251 & 1676350 & forward & tqs $A$ & -3231 & 0.33 & 152 & ydgH & 0.791 \\
\hline 52 & 2166386 & 2166485 & forward & cyaR & -1113 & 0.32 & 302 & yegs & 0.789 \\
\hline 53 & 2429322 & 2429421 & forward & folX,yfcH & -8650 & 0.28 & 6602 & flk & 0.789 \\
\hline 54 & 4083889 & 4083988 & forward & yiiF & -5848 & 0.29 & 102 & fdhD & 0.789 \\
\hline
\end{tabular}


Table 28 Top classification hits in E. coli uracil-comp. constrained (Continued)

\begin{tabular}{|c|c|c|c|c|c|c|c|c|c|}
\hline 55 & 2491327 & 2491426 & reverse & $y f d X$ & -413 & 0.32 & 99 & $\mathrm{frc}$ & 0.789 \\
\hline 56 & 1165025 & 1165124 & reverse & comR & -2349 & 0.31 & 4299 & fhuE & 0.789 \\
\hline 57 & 1933126 & 1933225 & forward & purT & -2994 & 0.3 & 1502 & yebK & 0.787 \\
\hline 58 & 1204407 & 1204506 & reverse & stfE,tfaE & -3284 & 0.25 & 2299 & ymfK & 0.787 \\
\hline 59 & 1493112 & 1493211 & forward & $\operatorname{trg}$ & -929 & 0.32 & 152 & ydcJ & 0.786 \\
\hline 60 & 1714050 & 1714149 & forward & gstA & -995 & 0.31 & 3802 & slyB & 0.786 \\
\hline 61 & 1397615 & 1397714 & forward & ynaJ & -1920 & 0.32 & 5102 & abgR & 0.784 \\
\hline 62 & 5034 & 5133 & forward & thrA,thrB,thrC,thrL & 35 & 0.27 & 152 & yaax & 0.784 \\
\hline 63 & 4547775 & 4547874 & reverse & gntP & -152 & 0.25 & 10299 & nanC,nanM & 0.784 \\
\hline 64 & 2257385 & 2257484 & forward & yeiL & -3300 & 0.3 & 4452 & setB & 0.783 \\
\hline 65 & 660791 & 660890 & forward & tatE & -2369 & 0.29 & 13402 & ybel & 0.781 \\
\hline 66 & 3945051 & 3945150 & reverse & hdfR & -51 & 0.29 & 5749 & hsrA,yieP & 0.781 \\
\hline 67 & 2238382 & 2238481 & forward & preA,preT & -3811 & 0.33 & 3502 & yeiG & 0.779 \\
\hline 68 & 925014 & 925113 & reverse & serW & -44 & 0.31 & 3249 & cspD & 0.779 \\
\hline 69 & 13587 & 13686 & reverse & hokC,mokC & -3115 & 0.3 & 1849 & yaal & 0.779 \\
\hline 70 & 2734984 & 2735083 & reverse & arof,tyrA & -1937 & 0.33 & 999 & rluD,yfiH & 0.778 \\
\hline 71 & 2880686 & 2880785 & forward & iap & -4997 & 0.32 & 9502 & queD & 0.777 \\
\hline 72 & 1078128 & 1078227 & forward & rutR & -3976 & 0.29 & 352 & putP & 0.776 \\
\hline 73 & 187962 & 188061 & forward & cdaR & -4293 & 0.32 & 1702 & rpsB,tff,tsf & 0.776 \\
\hline 74 & 497037 & 497136 & reverse & aes & -1152 & 0.25 & 7049 & pric,ybaM & 0.776 \\
\hline 75 & 3181662 & 3181761 & forward & zupT & -268 & 0.3 & 1152 & yqiC & 0.775 \\
\hline 76 & 593123 & 593222 & forward & appY & -9421 & 0.29 & 1652 & cusA,cusB,cusC,cusF & 0.775 \\
\hline 77 & 1250189 & 1250288 & forward & ycgY & -5317 & 0.33 & 52 & dhaR & 0.775 \\
\hline 78 & 3597882 & 3597981 & reverse & rpoH & -21 & 0.32 & 249 & livJ & 0.775 \\
\hline 79 & 117883 & 117982 & forward & guaC & -3347 & 0.33 & 802 & ampD,ampE & 0.774 \\
\hline 80 & 1073265 & 1073364 & forward & ymdF & -5739 & 0.31 & 152 & rutR & 0.774 \\
\hline 81 & 3416188 & 3416287 & reverse & alaU,ileU,rrfD, rrff, rrlD, rrsD, thrV & -5208 & 0.28 & 4749 & envR & 0.774 \\
\hline 82 & 4238098 & 4238197 & forward & yjbE,yjbF,yjbG,yjbH & -296 & 0.33 & 202 & psiE & 0.773 \\
\hline
\end{tabular}


Table 28 Top classification hits in E. coli uracil-comp. constrained (Continued)

\begin{tabular}{|c|c|c|c|c|c|c|c|c|c|}
\hline 83 & 3313859 & 3313958 & forward & psrO & -4390 & 0.32 & 2752 & $\operatorname{argG}$ & 0.773 \\
\hline 84 & 238253 & 238352 & reverse & yafU & -444 & 0.29 & 2299 & rnhA & 0.773 \\
\hline 85 & 2627711 & 2627810 & reverse & guaA,guaB & -1220 & 0.31 & 799 & $y f g F$ & 0.773 \\
\hline 86 & 4156263 & 4156362 & forward & $\operatorname{argB}, \operatorname{argC}, \operatorname{argH}$ & 32 & 0.3 & 202 & oxyR & 0.772 \\
\hline 87 & 58274 & 58373 & forward & $\operatorname{dj} \mid \mathrm{A}$ & -46 & 0.32 & 152 & yabP,yabe & 0.772 \\
\hline 88 & 3108528 & 3108627 & reverse & yghD,yghE & -35 & 0.33 & 1399 & speC & 0.772 \\
\hline 89 & 2750731 & 2750830 & reverse & ratA,ratB & -1250 & 0.31 & 2049 & grpE & 0.772 \\
\hline 90 & 454057 & 454156 & forward & bolA & 5 & 0.32 & 252 & tig & 0.771 \\
\hline 91 & 573621 & 573720 & forward & ybcQ & -10 & 0.26 & 2952 & essD, rrrD, rzpD & 0.771 \\
\hline 92 & 905496 & 905595 & forward & amiD,ybjQ & -481 & 0.31 & 10152 & ybjD & 0.771 \\
\hline 93 & 2407114 & 2407213 & reverse & yfbS & -379 & 0.32 & 2499 & $\operatorname{Irh} A$ & 0.771 \\
\hline 94 & 1733426 & 1733525 & reverse & ydhP & -670 & 0.31 & 1349 & grxD & 0.771 \\
\hline 95 & 604509 & 604608 & forward & pheP & -1902 & 0.33 & 2502 & hokE & 0.77 \\
\hline 96 & 1431895 & 1431994 & forward & lomR_2,stfR,tfaR & -836 & 0.31 & 3202 & micC & 0.77 \\
\hline 97 & 1395289 & 1395388 & forward & insH-4 & -124 & 0.28 & 52 & ynaJ & 0.769 \\
\hline 98 & 253317 & 253416 & forward & $\operatorname{din} B, y a f N$,yafO,yafP & -107 & 0.3 & 102 & prfH,ykfJ & 0.768 \\
\hline 99 & 573571 & 573670 & forward & ybcQ & 40 & 0.28 & 3002 & essD, rrrD, rzpD & 0.768 \\
\hline 100 & 3986826 & 3986925 & forward & glmZ & -2151 & 0.32 & 2302 & cyaA & 0.768 \\
\hline
\end{tabular}

Top 50 hits of the forward and reverse strands of the E. coli intergenic regions that have uracil composition within the range of known riboswitches in $E$. coli (between 0.23 and 0.34 ). $50 \mathrm{nt}-$ overlap $100 \mathrm{nt}$ window used. The ranking of each hit is denoted in column R. Distance from upstream and downstream operons are the distance from the center of the hit to the stop and start codons of upstream and downstream operons, respectively. Probability denotes the multinomial regression likelihood of being a riboswitch under the LMFEGCRND model. Positions are according to gbU00096.2 version of E. coli and not gbU00096.3 version.

'Table 28: Complete list of genes in this operon is nuoA,nuoB,nuoC,nuoE,nuoF,nuoG,nuoH,nuol,nuoJ,nuoK,nuoL,nuoM, nuoN. ${ }^{2}$ Table 28: Complete list of genes in this operon is yafW, yafX,yaff,ykfB,ykfF,ykfG,ykfH,ykf. Interesting hits are shown in bold. 
Table 29 Top entropy hits of $E$. coli filtered for GC- and uracil-comp

E. coli Start End Strand Upstream operon Dist. to upstream MFE MFE p. Val. GC $\quad$ RND RND p. Val. Uracil Dist. to downstream Downstream operon Probability

\begin{tabular}{lllllllllllll}
\hline $100 \mathrm{nt} 4083889$ & 4083988 forward & yiif & -5848 & -38.4 & 0.0267 & 0.53 & 58.6367989 & 0.0365 & 0.29 & 102 & fdhD & 0.789
\end{tabular}

$100 \mathrm{nt} 187962188061$ forward $\quad$ cdaR $\quad-4293 \quad-36.4 \quad 0.0466$

$100 \mathrm{nt} 952485952584$ forward

100 nt 41150384115137 forward

$\begin{array}{cccc}\text { ycak } & -2955 & -36.8 & 0.0419 \\ \text { uspD,yiis } & -3245 & -37 & 0.0396\end{array}$

$0.53 \quad 59.0985985$

$0.52 \quad 58.3203011$

0.0229

0.32
0.27

$0.53 \quad 58.3563995$

0.0477

0.33

1702

rpsB,tff,tsf

0.776

E. coli Start End Strand Upstream operon Dist. to upstream MFE MFE p. Val. GC RND RND p. Val. Uracil Dist. to downstream Downstream operon Probability

150 nt 26869232687072 forward

$\begin{array}{cccc}\text { hmp } & -1802 & -56.00 & - \\ \text { iap } & -11672 & -56.40 & - \\ \text { gspO' }^{1} & -2871 & -56.10 & - \\ \text { yhhW } & -74 & -55.60 & - \\ \text { yehS } & -13808 & -58.00 & -\end{array}$

$\begin{array}{llll}0.5333 & 90.7522964 & 0.0077 & 0.32000\end{array}$

$0.533389 .1240005 \quad-\quad 0.0294$

$\begin{array}{llll}0.5200 & 88.5419006 & 0.0450 & 0.29333\end{array}$

$\begin{array}{llll}0.4800 & 88.6371994 & 0.0419 & 0.30666\end{array}$

$0.533388 .6897964 \quad 0.0405 \quad 0.27333$

6827

2777

mltF

0.8671584129

150 nt 28873862887535 forward

150 nt 35768253576974 reverse

150 nt 21958662196015 reverse

$0.0405-0.27333 \quad 3749$

149

queD $\quad 0.8254097700$

slyX $\quad 0.8172816634$

gntK,gntR,gntU $\quad 0.8547886610$

Significant hits of the forward and reverse strands of the E. coli intergenic regions having significantly high RND entropy ( $\mathrm{p}$-Val. $<0.0500$ ), significantly low (p.Val.<0.050), GC and uracil compositions within the range of those for known riboswitches Threshold values and their corresponding p-values have been calculated separately for each scan (66414 segments). Distance from Upstream and Downstream or multino operon. Columns Upsream/Downstream operon show gene ID within the operon.

${ }^{1}$ Table 29: Complete list of genes in this operon is gspC,gspD,gspE,gspF,gspG,gspH,gspl,gspJ,gspK,gspL,gspM,gspO. 
Table 30 Top entropy hits in E. coli

E. coli Start End Strand Upstream operon Dist. to upstream MFE MFE p.Val. GC RND RND p. Val. Uracil Dist. to downstream Downstream operon Probability

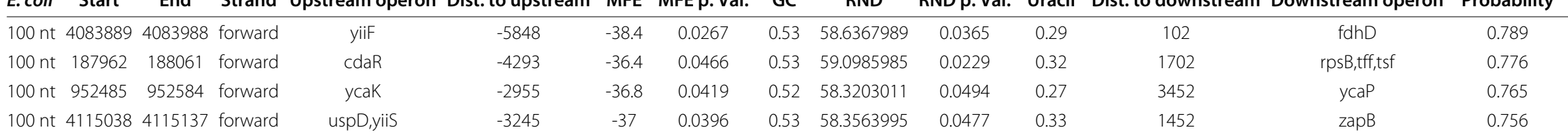

E. coli Start End Strand Upstream operon Dist. to upstream MFE MFE p. Val. GC RND RND p. Val. Uracil Dist. to downstream Downstream operon Probability

150 nt 26869232687072 forward

$150 \mathrm{nt} 452721452870$ forward

$150 \mathrm{nt} 11006991100848$ forward

150 nt 28873862887535 forward

150 nt 34671873467336 forward

150 nt 25531182553267 forward

150 nt 26602642660413 forward

150 nt 17667981766947 forward

150 nt 17183741718523 forward

150 nt 43567124356861 forward

$150 \mathrm{nt} 149580149729$ forward

150 nt 46044764604625 forward

150 nt 31200693120218 forward

150 nt 19820241982173 forward

150 nt 39218783922027 forward

$150 \mathrm{nt} 790921791070$ forward

$150 \mathrm{nt} 44822914482440$ forward

$150 \mathrm{nt} 518357518506$ forward

$150 \mathrm{nt} 11675461167695$ forward

150 nt 16424961642645 forward

150 nt 39161033916252 forward

\begin{tabular}{ccc} 
Upstream operon & Dist. to upstream & MFE \\
\hline hmp & -1797 & -56.00 \\
yajQ & -8244 & -60.90 \\
ycdZ & -610 & -58.70 \\
iap & -11667 & -56.40 \\
gspO' & -2866 & -56.10 \\
amiA,hemF & -893 & -57.40 \\
ryfA & -8005 & -56.90 \\
Ipp & -11038 & -57.00 \\
slyB & 72 & -58.70 \\
yjdK,yjdO & -5529 & -58.80 \\
yadD & -1631 & -57.80 \\
yjjZ & -334 & -57.20 \\
C0719 & -389 & -56.40 \\
uspC & -3740 & -56.20 \\
cbrB,cbrC & -25167 & -56.00 \\
aroG & -4934 & -57.00 \\
yjgN & -3263 & -58.30 \\
ybbL,ybbM & -1692 & -57.10 \\
ycfJ & -106 & -57.70 \\
cspF & -2326 & -56.00 \\
cbrB,cbrC & -19392 & -60.90
\end{tabular}

$\begin{array}{cc}\text { GC } & \text { RND } \\ 0.5333 & 90.7522964 \\ 0.5200 & 88.2920990 \\ 0.4933 & 87.8781967 \\ 0.5333 & 89.1240005 \\ 0.5200 & 88.5419006 \\ 0.5133 & 87.6467972 \\ 0.5000 & 87.1239014 \\ 0.4800 & 85.6548004 \\ 0.4867 & 85.1240005 \\ 0.5200 & 86.0333023 \\ 0.4600 & 84.3807983 \\ 0.4867 & 85.4507980 \\ 0.5267 & 87.1032028 \\ 0.5333 & 87.3750000 \\ 0.4933 & 85.5883026 \\ 0.5333 & 86.4469986 \\ 0.5200 & 85.4577026 \\ 0.5000 & 85.1729965 \\ 0.5333 & 85.9982986 \\ 0.5333 & 86.4957962 \\ 0.5200 & 84.0416031\end{array}$

0.0077

$0.32000 \quad 6822$

0.23333

0.30666

6822
897

2397

$0.0294 \quad 0.31333$

0.29333

0.28666

0.32000

0.25333

0.32666

0.26000

0.30666

0.27333

0.27333

0.26666

0.30666

0.28000

0.28666

0.26000

0.27333

0.25333

0.30000

2772

8397

3597

1122

222

597

9897

12447

1272

6147

2847

3222

2997

1872

522

672

1347

8997

$\begin{array}{cl}\text { mltF } & 0.8671584129 \\ \text { bolA } & 0.8664909005 \\ \text { sgA, csgB,csgC } & 0.8559710383 \\ \text { queD } & 0.8254097700 \\ \text { slyX } & 0.8172816634 \\ \text { intZ } & 0.8125300407 \\ \text { suhB } & 0.8031908870 \\ \text { ydiK } & 0.7757616639 \\ \text { dhl,ydhJ,ydhK } & 0.7731205821 \\ \text { fxsA } & 0.7661048174 \\ \text { hrpB } & 0.7651519775 \\ \text { holD,riml,yjjG } & 0.7621335387 \\ \text { glcC } & 0.7610746622 \\ \text { ftnB } & 0.7596676350 \\ \text { asnA } & 0.7384917736 \\ \text { acrZ } & 0.7345629930 \\ \text { lptF,lptG } & 0.7340587974 \\ \text { ybbA,ybbP } & 0.7300664783 \\ \text { bhsA } & 0.7274026275 \\ \text { ydfV } & 0.7190257311 \\ \text { asnA } & 0.7181233764\end{array}$


Table 30 Top entropy hits in E. coli (Continued)

\begin{tabular}{|c|c|c|c|c|c|c|c|c|c|c|c|c|c|c|}
\hline $150 \mathrm{nt}$ & 3258127 & 3258276 & forward & yhaK,yhaL & -4819 & -55.70 & - & 0.5267 & 86.1580963 & - & 0.26000 & 7197 & $\operatorname{tdcR}$ & 0.7085512877 \\
\hline $150 \mathrm{nt}$ & 3721360 & 3721509 & forward & insk & -1209 & -57.70 & - & 0.5133 & 84.8648987 & - & 0.26000 & 2472 & wecH & 0.7070741653 \\
\hline $150 \mathrm{nt}$ & 2438211 & 2438360 & forward & flk & -1165 & -58.50 & - & 0.5267 & 84.9561996 & - & 0.24000 & 1497 & $\mathrm{mnmC}$ & 0.7053987384 \\
\hline $150 \mathrm{nt}$ & 1268246 & 1268395 & forward & kdsA,ychA,ychQ & 75 & -56.90 & - & 0.4933 & 84.3839035 & - & 0.29333 & 222 & rdlA & 0.7012539506 \\
\hline $150 \mathrm{nt}$ & 219458 & 219607 & forward & arfB,nlpE,yaeQ & -3400 & -59.90 & - & 0.5267 & 84.2009964 & - & 0.27333 & 3297 & gmhB & 0.6966923475 \\
\hline $150 \mathrm{nt}$ & 3514668 & 3514817 & forward & frlA,frlB,frlC,frlD,frlR & -11784 & -56.50 & - & 0.5333 & 85.6490021 & - & 0.27333 & 6147 & mrcA & 0.6895118356 \\
\hline $150 \mathrm{nt}$ & 3313884 & 3314033 & forward & $\mathrm{psrO}$ & -4385 & -55.60 & - & 0.5067 & 84.9284973 & - & 0.28666 & 2697 & $\operatorname{argG}$ & 0.6809250712 \\
\hline $150 \mathrm{nt}$ & 649808 & 649957 & forward & $y b d R$ & -7180 & -58.10 & - & 0.5333 & 84.6990967 & - & 0.25333 & 1572 & dpiA,dpiB & 0.6750817299 \\
\hline $150 \mathrm{nt}$ & 2243989 & 2244138 & forward & yeiG & -1142 & -57.10 & - & 0.5333 & 84.3582993 & - & 0.26000 & 3672 & yeiH & 0.6381362677 \\
\hline $150 \mathrm{nt}$ & 4253685 & 4253834 & forward & ubiA,ubiC & -1695 & -55.80 & - & 0.5267 & 84.4711990 & - & 0.29333 & 897 & dgkA & 0.6285824776 \\
\hline $150 \mathrm{nt}$ & 2866503 & 2866652 & forward & ygbN & -1937 & -56.80 & - & 0.5333 & 84.2586975 & - & 0.30000 & 8022 & iap & 0.6268372536 \\
\hline $150 \mathrm{nt}$ & 3576825 & 3576974 & reverse & yhhW & -69 & -55.60 & - & 0.4800 & 88.6371994 & 0.0419 & 0.30666 & 154 & gntK,gntR,gntU & 0.8547886610 \\
\hline $150 \mathrm{nt}$ & 260750 & 260899 & reverse & yafW,yafX,yafY,ykfB,ykfF,ykfG,ykfH,ykfl & -1723 & -62.00 & - & 0.5267 & 86.8554001 & - & 0.32000 & 1504 & phoE & 0.8323625326 \\
\hline $150 \mathrm{nt}$ & 2195866 & 2196015 & reverse & yehs & -13803 & -58.00 & - & 0.5333 & 88.6897964 & 0.0405 & 0.27333 & 3754 & $\mathrm{mrp}$ & 0.8320623040 \\
\hline $150 \mathrm{nt}$ & 4176725 & 4176874 & reverse & $\mathrm{sroH}$ & -11546 & -58.80 & - & 0.5333 & 88.1481018 & - & 0.28000 & 3754 & coaA & 0.8252514601 \\
\hline $150 \mathrm{nt}$ & 133211 & 133360 & reverse & speD,speE,yacC & -1498 & -56.40 & - & 0.5133 & 88.0559998 & - & 0.28000 & 2029 & yacH & 0.8126859665 \\
\hline $150 \mathrm{nt}$ & 44332 & 44481 & reverse & apaG,apaH,IptD,pdxA,rsmA,surA & -5969 & -64.00 & - & 0.5333 & 85.3160019 & - & 0.26000 & 2479 & caiA,caiB,caiC,caiD,caiE,caiT & 0.8009238243 \\
\hline $150 \mathrm{nt}$ & 2248916 & 2249065 & reverse & nupX & -1922 & -55.70 & - & 0.5333 & 88.3962021 & - & 0.28000 & 1354 & yeiE & 0.7910096645 \\
\hline $150 \mathrm{nt}$ & 2774331 & 2774480 & reverse & ypjA & -1758 & -55.60 & - & 0.5200 & 87.4978027 & - & 0.26666 & 3229 & ypjM_1,ypjM_2 & 0.7727379203 \\
\hline $150 \mathrm{nt}$ & 2184269 & 2184418 & reverse & yehA,yehB,yehC,yehD & -1054 & -56.20 & - & 0.5333 & 87.6942978 & - & 0.30666 & 529 & $\mathrm{rcnR}$ & 0.7722578049 \\
\hline $150 \mathrm{nt}$ & 1655707 & 1655856 & reverse & mlc,ynfK & -8762 & -58.20 & - & 0.5267 & 86.6440964 & - & 0.24000 & 304 & ynfC & 0.7716723680 \\
\hline $150 \mathrm{nt}$ & 2168389 & 2168538 & reverse & gatR_2 & -951 & -56.70 & - & 0.5133 & 86.7958984 & - & 0.31333 & 304 & gatR_1 & 0.7715415359 \\
\hline $150 \mathrm{nt}$ & 3481959 & 3482108 & reverse & yhfA & -1398 & -57.90 & - & 0.5333 & 86.7799988 & - & 0.25333 & 2854 & kefB,kefG,yheV & 0.7633723617 \\
\hline $150 \mathrm{nt}$ & 314642 & 314791 & reverse & ykgA & -953 & -58.20 & - & 0.5067 & 85.1943970 & - & 0.32000 & 2254 & ykgR & 0.7403761148 \\
\hline $150 \mathrm{nt}$ & 2783559 & 2783708 & reverse & ileY & -146 & -56.20 & - & 0.4667 & 84.4744034 & - & 0.26666 & 604 & yрjC & 0.7329583764 \\
\hline $150 \mathrm{nt}$ & 4050403 & 4050552 & reverse & $g \ln A, g \ln G, g \mid n L$ & -1410 & -56.70 & - & 0.5267 & 86.2409973 & - & 0.27333 & 1729 & yihA & 0.7301918864 \\
\hline $150 \mathrm{nt}$ & 2465055 & 2465204 & reverse & yfdK,yfdL,yfdM,yfdN,yfdO & -3965 & -58.50 & - & 0.5333 & 85.6035004 & - & 0.25333 & 2104 & mlaA & 0.7242872119 \\
\hline $150 \mathrm{nt}$ & 2809717 & 2809866 & reverse & luxs & -2444 & -56.20 & - & 0.5333 & 86.4486008 & - & 0.29333 & 11779 & ygaC & 0.7205215693 \\
\hline
\end{tabular}


Table 30 Top entropy hits in E. coli (Continued)

\begin{tabular}{|c|c|c|c|c|c|c|c|c|c|c|c|c|c|c|}
\hline $150 \mathrm{nt}$ & 3341463 & 3341612 & reverse & elbB,mtgA & -5561 & -57.50 & - & 0.5000 & 84.6742020 & - & 0.28000 & 3454 & $\mathrm{mlaB}, \mathrm{mlaC}, \mathrm{mlaD}$,mlaE,mlaF & 0.7152099609 \\
\hline $150 \mathrm{nt}$ & 1950122 & 1950271 & reverse & torY,torZ & -2401 & -56.44 & - & 0.5200 & 85.7341003 & - & 0.24666 & 1654 & asps & 0.7131192684 \\
\hline $150 \mathrm{nt}$ & 3059434 & 3059583 & reverse & ygfl & -4786 & -56.20 & - & 0.4933 & 84.9087982 & - & 0.27333 & 1879 & yqfE & 0.7122740149 \\
\hline $150 \mathrm{nt}$ & 2530006 & 2530155 & reverse & $\mathrm{pdxk}$ & -4323 & -58.20 & - & 0.5000 & 84.2724991 & - & 0.25333 & 829 & zipA & 0.7098694444 \\
\hline $150 \mathrm{nt}$ & 757742 & 757891 & reverse & mngR & -6555 & -57.90 & - & 0.4867 & 83.9291000 & - & 0.31333 & 4129 & gltA & 0.7092900276 \\
\hline $150 \mathrm{nt}$ & 4452551 & 4452700 & reverse & $\mathrm{fbp}$ & -4 & -56.90 & - & 0.5200 & 85.3691025 & - & 0.29333 & 4954 & ppa & 0.7049999237 \\
\hline $150 \mathrm{nt}$ & 3584997 & 3585146 & reverse & ugpA,ugpB,ugpC,ugpE,ugpQ & -317 & -59.30 & - & 0.5133 & 84.1650009 & - & 0.32000 & 229 & ggt & 0.7046704292 \\
\hline $150 \mathrm{nt}$ & 3459796 & 3459945 & reverse & bfd,bfr & -4396 & -59.60 & - & 0.5200 & 84.1896973 & - & 0.23333 & 6454 & gspA,gspB & 0.7009978294 \\
\hline $150 \mathrm{nt}$ & 3395856 & 3396005 & reverse & mreB,mreC,mreD & -474 & -56.20 & - & 0.5000 & 84.8627014 & - & 0.25333 & 1654 & yhdP & 0.6998521686 \\
\hline $150 \mathrm{nt}$ & 2321601 & 2321750 & reverse & yfaA,yfaP,yfaQ,yfaS_1,yfaS_2,yfaT & -3709 & -61.10 & - & 0.5333 & 83.9000015 & - & 0.24000 & 3829 & $\operatorname{rcs} C$ & 0.6947578788 \\
\hline $150 \mathrm{nt}$ & 4169919 & 4170068 & reverse & coaA & -2101 & -56.20 & - & 0.4933 & 84.4697037 & - & 0.26000 & 8704 & $\operatorname{trmA}$ & 0.6920779943 \\
\hline $150 \mathrm{nt}$ & 4029970 & 4030119 & reverse & mobA,mobB & -8880 & -57.80 & - & 0.5133 & 84.4968033 & - & 0.30000 & 1054 & fadA, fadB & 0.6919249892 \\
\hline $150 \mathrm{nt}$ & 2366493 & 2366642 & reverse & pmrD & -4722 & -56.00 & - & 0.5200 & 85.1843033 & - & 0.28000 & 2929 & ais & 0.6792802215 \\
\hline $150 \mathrm{nt}$ & 1850076 & 1850225 & reverse & $y d j E$ & -490 & -57.40 & - & 0.5267 & 84.6417999 & - & 0.27333 & 3454 & selD,topB,ydjA & 0.6695327163 \\
\hline $150 \mathrm{nt}$ & 3246739 & 3246888 & reverse & yhaJ & -4522 & -56.00 & - & 0.4867 & 83.8035965 & - & 0.26000 & 4054 & uxaA,uxaC & 0.6671289802 \\
\hline $150 \mathrm{nt}$ & 3883703 & 3883852 & reverse & purP & -9513 & -56.60 & - & 0.5200 & 84.5989990 & - & 0.28000 & 2029 & dnaA,dnaN,recF & 0.6626442075 \\
\hline $150 \mathrm{nt}$ & 4395241 & 4395390 & reverse & yjfN & -18720 & -56.20 & - & 0.5267 & 84.9857025 & - & 0.29333 & 3229 & queG & 0.6626062393 \\
\hline $150 \mathrm{nt}$ & 2664571 & 2664720 & reverse & hсaT & -79 & -56.20 & - & 0.5267 & 84.7899017 & - & 0.24666 & 3304 & trmJ & 0.6529098153 \\
\hline $150 \mathrm{nt}$ & 2975533 & 2975682 & reverse & lysA & -47 & -57.20 & - & 0.5333 & 84.5955963 & - & 0.24666 & 1204 & omrB & 0.6521243453 \\
\hline $150 \mathrm{nt}$ & 796718 & 796867 & reverse & ybhA & -39 & -58.40 & - & 0.5333 & 83.8794022 & - & 0.26666 & 2929 & modE,modF & 0.6405209899 \\
\hline $150 \mathrm{nt}$ & 3288088 & 3288237 & reverse & rsml & -2330 & -55.90 & - & 0.5067 & 83.9057007 & - & 0.28000 & 11479 & agaR & 0.6363855004 \\
\hline $150 \mathrm{nt}$ & 3211791 & 3211940 & reverse & mug & -1119 & -56.10 & - & 0.5200 & 84.1829987 & - & 0.25333 & 3304 & tsaD & 0.6315983534 \\
\hline $150 \mathrm{nt}$ & 2161503 & 2161652 & reverse & ogrk & -3744 & -55.70 & - & 0.5333 & 84.3114014 & - & 0.29333 & 10429 & yegK,yegL & 0.6064385772 \\
\hline $150 \mathrm{nt}$ & 3284413 & 3284562 & reverse & $\mathrm{rsml}$ & -6005 & -55.60 & - & 0.5333 & 84.2777023 & - & 0.25333 & 7804 & agaR & 0.6025381684 \\
\hline
\end{tabular}

Significant hits of the forward and reverse strands of the $E$. coli intergenic regions having high RND entropy ( $p$-Val. $<0.500$ ), significantly low ( $p . V a l$. $<0.050)$, GC and uracil compositions within the range of those for known riboswitches Threshold values and their corresponding p-values have been calculated separately for each genome-wide test. $50 \mathrm{nt}$ overlap used for $100 \mathrm{nt}$ scan (100090 segments). $175 \mathrm{nt}$ overlap used for $150 \mathrm{nt}$ scan ( 66414 regression likelihood of being a riboswitch under the LMFEGCRND model. Positions are according to gbU00096.2 version of $E$. coli and not gbU00096.3 version. Negative values indicate distance to upstream operon. Columns Upsream/Downstream Operon show gene ID within the operon.

${ }^{1}$ Table 30: Complete list of genes in this operon is gspC,gspD,gspE,gspF,gspG,gspH,gspl,gspJ,gspK,gspL,gspM,gspO. 


\section{Table 31 Top classification hits in S. elongatus}

\begin{tabular}{|c|c|c|c|c|c|c|c|c|c|}
\hline $\mathbf{R}$ & Start & End & Strand & $\begin{array}{l}\text { Upstream } \\
\text { operon }\end{array}$ & $\begin{array}{l}\text { Dist. to } \\
\text { upstream }\end{array}$ & Uracil & $\begin{array}{l}\text { Dist. to } \\
\text { downstream }\end{array}$ & $\begin{array}{l}\text { Downstream } \\
\text { operon }\end{array}$ & Probability \\
\hline 1 & 2239899 & 2240048 & forward & psbV & -2288 & 0.34666 & 1277 & coaD & 0.9169 \\
\hline 2 & 1788275 & 1788424 & reverse & syc1657_c & -1875 & 0.19333 & 5099 & syc1649_c & 0.9156 \\
\hline 3 & 1673354 & 1673503 & forward & $\mathrm{accB}$ & -811 & 0.22666 & 3377 & $\operatorname{des} C$ & 0.9078 \\
\hline 4 & 734659 & 734808 & forward & syc0660_d & -635 & 0.24666 & 8177 & syc0665_d & 0.9004 \\
\hline 5 & 1108388 & 1108537 & reverse & lacF & -1124 & 0.41333 & 1124 & $\operatorname{dev} B$ & 0.8967 \\
\hline 6 & 838999 & 839148 & forward & gst1 & -2009 & 0.20666 & 2552 & hisG & 0.8906 \\
\hline 7 & 734734 & 734883 & forward & syc0660_d & -710 & 0.28 & 8102 & syc0665_d & 0.886 \\
\hline 8 & 1674179 & 1674328 & forward & $\mathrm{accB}$ & -1636 & 0.28 & 2552 & $\operatorname{des} C$ & 0.8857 \\
\hline 9 & 936254 & 936403 & forward & syc0839_d & -190 & 0.26 & 77 & syc0840_d & 0.8855 \\
\hline 10 & 1706450 & 1706599 & forward & syc1578_d & -2580 & 0.28 & 5252 & gpsA & 0.8834 \\
\hline 11 & 2686971 & 2687120 & reverse & syc2516_c & -462 & 0.24666 & 674 & ycf49 & 0.8809 \\
\hline 12 & 464795 & 464944 & forward & syc0415_d & -1330 & 0.46 & 377 & syc0416_d & 0.8784 \\
\hline 13 & 546798 & 546947 & reverse & syc0485_c & -190 & 0.26666 & 74 & syc0484_c & 0.8778 \\
\hline 14 & 1527281 & 1527430 & reverse & holB & -916 & 0.34 & 6674 & syc1405_c & 0.8749 \\
\hline 15 & 2251075 & 2251224 & reverse & masA & -985 & 0.25333 & 674 & syc2098_c & 0.8741 \\
\hline 16 & 590779 & 590928 & reverse & syc0539_c & -11831 & 0.2 & 2624 & syc0528_c & 0.8733 \\
\hline 17 & 1870479 & 1870628 & forward & syc1725_d & -722 & 0.21333 & 5102 & syc1732_d & 0.873 \\
\hline 18 & 2098418 & 2098567 & forward & sui1 & -7782 & 0.27333 & 2252 & dapB & 0.8715 \\
\hline 19 & 793922 & 794071 & reverse & asns & -1542 & 0.46666 & 224 & syc0707_c & 0.8703 \\
\hline 20 & 1218955 & 1219104 & reverse & $\operatorname{trpF}$ & -430 & 0.34 & 1799 & sycRNA024_c & 0.8703 \\
\hline 21 & 1751997 & 1752146 & reverse & syc1625_c & -3772 & 0.32666 & 3899 & syc1619_c & 0.8691 \\
\hline 22 & 1526981 & 1527130 & reverse & holB & -1216 & 0.32 & 6374 & syc1405_c & 0.8686 \\
\hline 23 & 1120163 & 1120312 & reverse & syc1011_c & -4710 & 0.22 & 3749 & syc1002_c & 0.8684 \\
\hline 24 & 69102 & 69251 & reverse & syc0062_c & -2424 & 0.18666 & 974 & syc0058_c & 0.8668 \\
\hline 25 & 132050 & 132199 & reverse & $\operatorname{argC}$ & -633 & 0.21333 & 7724 & syc0114_c & 0.8668 \\
\hline 26 & 1348202 & 1348351 & reverse & syc1238_c & -2618 & 0.22 & 1049 & syc1235_c & 0.8666 \\
\hline
\end{tabular}


Table 31 Top classification hits in S. elongatus (Continued)

\begin{tabular}{|c|c|c|c|c|c|c|c|c|c|}
\hline 27 & 1674104 & 1674253 & forward & $\mathrm{accB}$ & -1561 & 0.26666 & 2627 & $\operatorname{des} C$ & 0.8663 \\
\hline 28 & 479806 & 479955 & forward & syc0423_d & -3318 & 0.34666 & 302 & syc0428_d & 0.8656 \\
\hline 29 & 2009813 & 2009962 & forward & syc1856_d & 36 & 0.29333 & 1352 & syc1859_d & 0.8647 \\
\hline 30 & 1674554 & 1674703 & forward & $\mathrm{accB}$ & -2011 & 0.27333 & 2177 & desC & 0.8646 \\
\hline 31 & 1372776 & 1372925 & reverse & syc1262_c & -154 & 0.28666 & 824 & gyrA & 0.863 \\
\hline 32 & 702997 & 703146 & forward & syc0629_d & -3402 & 0.27333 & 3452 & $a c n B$ & 0.8622 \\
\hline 33 & 1673054 & 1673203 & forward & $\mathrm{accB}$ & -511 & 0.29333 & 3677 & $\operatorname{des} C$ & 0.8622 \\
\hline 34 & 971765 & 971914 & reverse & priA & -1871 & 0.3 & 1199 & psbDl & 0.861 \\
\hline 35 & 2574891 & 2575040 & forward & syc2417_d & -956 & 0.21333 & 6227 & syc2423_d & 0.8585 \\
\hline 36 & 2033882 & 2034031 & forward & prfA & 38 & 0.26 & 527 & alr & 0.8579 \\
\hline 37 & 2633236 & 2633385 & forward & syc2468_d & -1767 & 0.22666 & 902 & rps4 & 0.8555 \\
\hline 38 & 2031022 & 2031171 & reverse & mrcA & -2764 & 0.22 & 14249 & syc1864_c & 0.8549 \\
\hline 39 & 1706375 & 1706524 & forward & syc1578_d & -2505 & 0.29333 & 5327 & gpsA & 0.8543 \\
\hline 40 & 69327 & 69476 & reverse & syc0062_c & -2199 & 0.21333 & 1199 & syc0058_c & 0.8532 \\
\hline 41 & 2383320 & 2383469 & forward & syc2228_d & -2586 & 0.26 & 1052 & natB & 0.8531 \\
\hline 42 & 2162031 & 2162180 & forward & syc2015_d & -852 & 0.33333 & 5402 & pilB & 0.8527 \\
\hline 43 & 1848963 & 1849112 & forward & syc1711_d & -916 & 0.23333 & 6527 & syc1716_d & 0.8521 \\
\hline 44 & 437513 & 437662 & forward & syc0392_d & -5939 & 0.35333 & 677 & syc0396_d & 0.8509 \\
\hline 45 & 1108838 & 1108987 & reverse & lacF & -674 & 0.30666 & 1574 & $\operatorname{dev} B$ & 0.8508 \\
\hline 46 & 1763242 & 1763391 & forward & syc1627_d & -4972 & 0.26 & 5027 & aroE & 0.8504 \\
\hline 47 & 298041 & 298190 & reverse & syc0257_c & -92 & 0.40666 & 224 & syc0256_c & 0.8502 \\
\hline 48 & 1357802 & 1357951 & reverse & uppS & -635 & 0.22 & 749 & gidB & 0.8501 \\
\hline 49 & 1674704 & 1674853 & forward & $\mathrm{accB}$ & -2161 & 0.34666 & 2027 & $\operatorname{des} C$ & 0.8495 \\
\hline 50 & 2225318 & 2225467 & reverse & syc2077_c & -5809 & 0.26666 & 1724 & $\mathrm{rfaG}$ & 0.8493 \\
\hline 51 & 1967731 & 1967880 & reverse & amt1 & -197 & 0.26 & 974 & syc1819_c & 0.8483 \\
\hline 52 & 1108913 & 1109062 & reverse & lacF & -599 & 0.34666 & 1649 & $\operatorname{dev} B$ & 0.848 \\
\hline 53 & 2664277 & 2664426 & forward & syc2500_d & -688 & 0.24666 & 1502 & pcrA & 0.8477 \\
\hline 54 & 838924 & 839073 & forward & gst1 & -1934 & 0.18 & 2627 & hisG & 0.8469 \\
\hline
\end{tabular}


Table 31 Top classification hits in S. elongatus (Continued)

\begin{tabular}{|c|c|c|c|c|c|c|c|c|c|}
\hline 55 & 1996234 & 1996383 & reverse & sycRNA048_c & -2339 & 0.26 & 4799 & syc1843_c & 0.8453 \\
\hline 56 & 1674479 & 1674628 & forward & $\mathrm{accB}$ & -1936 & 0.29333 & 2252 & $\operatorname{des} \mathrm{C}$ & 0.8435 \\
\hline 57 & 1320549 & 1320698 & reverse & $s s b$ & -269 & 0.26 & 1349 & syc1209_c & 0.8421 \\
\hline 58 & 542490 & 542639 & forward & syc0480_d & -2611 & 0.28 & 19127 & syc0503_d & 0.8419 \\
\hline 59 & 1089898 & 1090047 & reverse & nhaS4 & -886 & 0.2 & 974 & syc0972_c & 0.8417 \\
\hline 60 & 2383395 & 2383544 & forward & syc2228_d & -2661 & 0.27333 & 977 & natB & 0.8416 \\
\hline 61 & 2201969 & 2202118 & reverse & syc2052_c & -3184 & 0.32666 & 3074 & gcvH & 0.8408 \\
\hline 62 & 2475474 & 2475623 & forward & syc2315_d & -359 & 0.28666 & 4427 & syc2322_d & 0.8399 \\
\hline 63 & 1910646 & 1910795 & forward & psaL & -4022 & 0.21333 & 752 & syc1766_d & 0.8391 \\
\hline 64 & 2244572 & 2244721 & forward & syc2089_d & -2066 & 0.26 & 902 & syc2094_d & 0.8378 \\
\hline 65 & 2202119 & 2202268 & reverse & syc2052_c & -3034 & 0.33333 & 3224 & $\mathrm{gcvH}$ & 0.8378 \\
\hline 66 & 979835 & 979984 & reverse & hemL & -205 & 0.31333 & 149 & syc0880_c & 0.8371 \\
\hline 67 & 2201519 & 2201668 & reverse & syc2052_c & -3634 & 0.32 & 2624 & gcvH & 0.8366 \\
\hline 68 & 2436792 & 2436941 & forward & htpG & -4101 & 0.35333 & 3227 & syc2285_d & 0.8358 \\
\hline 69 & 2379191 & 2379340 & forward & syc2225_d & -348 & 0.22666 & 977 & syc2228_d & 0.8347 \\
\hline 70 & 751797 & 751946 & forward & syc0670_d & -2744 & 0.20666 & 3527 & syc0679_d & 0.8335 \\
\hline 71 & 2488156 & 2488305 & forward & syc2327_d & -3334 & 0.24666 & 2402 & syc2333_d & 0.8333 \\
\hline 72 & 1099747 & 1099896 & forward & syc0978_d & -5042 & 0.31333 & 1727 & $\mathrm{psaC}$ & 0.8327 \\
\hline 73 & 316592 & 316741 & forward & moaA & -195 & 0.38 & 302 & syc0272_d & 0.8319 \\
\hline 74 & 979910 & 980059 & reverse & hemL & -130 & 0.29333 & 224 & syc0880_c & 0.8319 \\
\hline 75 & 636787 & 636936 & forward & prk & -916 & 0.22 & 1802 & syc0569_d & 0.8318 \\
\hline 76 & 1709000 & 1709149 & forward & syc1578_d & -5130 & 0.26666 & 2702 & gpsA & 0.8317 \\
\hline 77 & 888391 & 888540 & forward & syc0774_d & -22228 & 0.19333 & 14402 & syc0808_d & 0.8304 \\
\hline 78 & 542940 & 543089 & forward & syc0480_d & -3061 & 0.29333 & 18677 & syc0503_d & 0.8303 \\
\hline 79 & 1736496 & 1736645 & reverse & gyrB & -7265 & 0.24666 & 1724 & aroF & 0.83 \\
\hline 80 & 542265 & 542414 & forward & syc0480_d & -2386 & 0.28 & 19352 & syc0503_d & 0.8299 \\
\hline 81 & 482179 & 482328 & reverse & syc0432_c & -3508 & 0.2 & 2249 & rpl33 & 0.8299 \\
\hline 82 & 882016 & 882165 & forward & syc0774_d & -15853 & 0.2 & 20777 & syc0808_d & 0.8297 \\
\hline
\end{tabular}


Table 31 Top classification hits in S. elongatus (Continued)

\begin{tabular}{|c|c|c|c|c|c|c|c|c|c|}
\hline 83 & 2239824 & 2239973 & forward & psbV & -2213 & 0.32666 & 1352 & coaD & 0.8293 \\
\hline 84 & 55214 & 55363 & forward & syc0043_d & -1501 & 0.24666 & 4802 & syc0049_d & 0.8292 \\
\hline 85 & 707052 & 707201 & reverse & syc0639_c & -3061 & 0.22 & 824 & syc0636_c & 0.8292 \\
\hline 86 & 992221 & 992370 & reverse & syc0895_c & -5003 & 0.34 & 2099 & eno & 0.8289 \\
\hline 87 & 234289 & 234438 & reverse & syc0202_c & -357 & 0.38666 & 149 & syc0201_c & 0.828 \\
\hline 88 & 1674629 & 1674778 & forward & $\mathrm{accB}$ & -2086 & 0.29333 & 2102 & $\operatorname{des} C$ & 0.8278 \\
\hline 89 & 1788200 & 1788349 & reverse & syc1657_c & -1950 & 0.16 & 5024 & syc1649_c & 0.8273 \\
\hline 90 & 1084661 & 1084810 & reverse & $\mathrm{nfrC}$ & -959 & 0.21333 & 449 & syc0967_c & 0.8273 \\
\hline 91 & 131975 & 132124 & reverse & $\operatorname{argC}$ & -708 & 0.22 & 7649 & syc0114_c & 0.8271 \\
\hline 92 & 632779 & 632928 & reverse & petH & -327 & 0.32666 & 3599 & syc0562_c & 0.8269 \\
\hline 93 & 2612915 & 2613064 & reverse & syc2455_c & -5717 & 0.28666 & 2624 & syc2447_c & 0.8266 \\
\hline 94 & 539354 & 539503 & reverse & $\mathrm{pdhC}$ & -603 & 0.22666 & 3074 & syc0476_c & 0.8263 \\
\hline 95 & 2360335 & 2360484 & reverse & syc2221_c & -10564 & 0.19333 & 899 & rec」 & 0.8245 \\
\hline 96 & 503343 & 503492 & reverse & syc0452_c & -2439 & 0.26 & 1274 & syc0448_c & 0.8242 \\
\hline 97 & 621230 & 621379 & reverse & syc0558_c & -3271 & 0.20666 & 299 & syc0552_c & 0.8195 \\
\hline 98 & 2077186 & 2077335 & reverse & syc1942_c & -5748 & 0.18666 & 1349 & apcF & 0.8187 \\
\hline 99 & 971165 & 971314 & reverse & priA & -2471 & 0.28 & 599 & psbDI & 0.8163 \\
\hline 100 & 1555584 & 1555733 & reverse & syc1449_c & -12609 & 0.35333 & 8549 & рерP & 0.815 \\
\hline
\end{tabular}

Top 50 hits of the forward and reverse strands of the S. elongatus intergenic regions using $75 \mathrm{nt}$-overlap $150 \mathrm{nt}$ window and under the LMFEGCRND model. The ranking of each hit is denoted in column R. Distance from upstream and downstream operons are the distance from the center of the hit to the stop and start codons of upstream and downstream operons, respectively. Probability denotes the multinomial regression likelihood of being a riboswitch under the LMFEGCRND model. 


\section{Competing interests}

The authors declare that they have no competing interests.

\section{Authors' contributions}

AM performed the tests, organized the results and drafted the manuscript. JA conceived the overall framework and aided with the writing. Both authors read and approved the final manuscript.

\section{Acknowledgements}

This work was done under the research assistantship received from the institute of bioinformatics at the University of Georgia. We also would like to acknowledge the The Georgia Advanced Computing Resource Center (GACRC) and the NSF MRI - DBI 0821263 award for the cluster for supporting the work. This research was supported in part by the Intramural Research Program of the NIH, National Library of Medicine. We thank Dr. John L. Spouge for his support. We also thank anonymous reviewers for their time and critical suggestions which were subsequently incorporated in this work.

\section{Author details}

${ }^{1}$ Institute of Bioinformatics, University of Georgia, Davison Life Sciences Bldg, Room B118B, 120 Green St, 30602 Athens, USA. ${ }^{2}$ National Center for Biotechnology Information (NCBI), NIH, Building 38A, RM 6S614K, 8600 Rockville Pike, 20894 Bethesda, USA. ${ }^{3}$ Department of Genetics, University of Georgia, Davison Life Sciences Bldg, 120 Green St, 30602 Athens, USA.

Received: 31 July 2014 Accepted: 2 March 2015

Published online: 28 April 2015

\section{References}

1. Morris KV. RNA and the Regulation of gene expression : A hidden layer of complexity. Norfolk: Caister Academic Press; 2008.

2. Morris KV. Non-coding RNAs and Epigenetic Regulation of Gene Expression: Drivers of Natural Selection. The Scripps Research Institute, La Jolla, California, USA: Caister Academic Press; 2012.

3. Barrandon C, Spiluttini B, Bensaude O. Non-coding RNAs regulating the transcriptional machiner. Biol Cell. 2008;100:83-95.

4. Repoila F, Darfeuille F. Small regulatory non-coding RNAs in bacteria: physiology and mechanistic aspects. Biol Cell. 2009;101:117-31.

5. Hall MN, Gabay J, Debarbouille M, Schwartz M. A role for mRNA secondary structure in the control of translation initiation. Nature. 1982;295:616-8.

6. Simmonds P, Karakasiliotis I, Bailey D, Chaudhry Y, Evans DJ, Goodfellow IG. Bioinformatic and functional analysis of RNA secondary structure elements among different genera of human and animal caliciviruses. Nucleic Acids Res. 2008:36:2530-46.

7. Grundy FJ, Henkin TM. From ribosome to riboswitch: control of gene expression in bacteria by RNA structural rearrangements. Crit Rev Biochem Mol Biol. 2006;41(6):329-38.

8. Oppenheim DS, Yanofsky C. Translational coupling during expression of the tryptophan operon of Escherichia coli. Genetics. 1980;95(4):785-95.

9. Merino E, Yanofsky C. Transcription attenuation: a highly conserved regulatory strategy used by bacteria. Trends Genet. 2005;21(5):260-4.

10. Eddy SR. Non-coding RNA genes and the modern RNA world. Nat Rev Genet. 2001;2(12):919-29.

11. Yao Z, Weinberg Z, Ruzzo WL. Cmfinder-a covariance model based RNA motif finding algorithm. Bioinformatics. 2006;22(4):445-52. Using Smart Source Parsing Feb 15; Epub 2005 Dec 15

12. Gruber AR, Findeiss S, Washietl S, Hofacker IL, Stadler PF. 2.0: improved noncoding RNA detection. Pac Symp Biocomput. 201069-79. Using Smart Source Parsing.

13. Nawrocki EP, Eddy SR. Infernal 1.1: 100-fold faster rRNA homology searches. Bioinformatics. 2013;29(22):2933-5.

14. Schattner P, Brooks A. N, Lowe TM. The TRNAscan-SE, snoscan and snoGPS web servers for the detection of tRNAS and snoRNAS. Nucleic Acids Res. 2005;33(Web Server issue):686-9. Using Smart Source Parsing Jul 1.

15. Tempel S, Tahi F. A fast ab-initio method for predicting miRNA precursors in genomes. Nucleic Acids Res. 2012;40(11):22. Using Smart Source Parsing Jun;:e80. doi: 10.1093/nar/gks146. Epub 2012 Feb.

16. Leclercq M, Diallo AB, Blanchette M. Computational prediction of the localization of microRNAs within their pre-miRNA. Nucleic Acids Res. 2013;41(15):7200-11. Using Smart Source Parsing Aug; doi: 10.1093/nar/gkt466. Epub 2013 Jun 8.

17. Nechooshtan G. A pH-responsive riboregulator. Genes Dev. 2009;23(22):2650.

18. Cromie MJ, Shi Y, Latifi T, Groisman EA. An RNA sensor for intracellular mg(2+). Cell. 2006;125(1):71-84.

19. Dann RCE, Wakeman CA, Sieling CL, Baker SC, Irnov I, Winkler WC. Structure and mechanism of a metal-sensing regulatory RNA. Cell. 2007;130(5):878-92.

20. Hollands K. Riboswitch control of rho-dependent transcription termination. Proc Natl Acad Sci U S A. 2012;109(14): 5376.

21. Nocker A, Hausherr T, Balsiger S, Krstulovic NP, Hennecke H, Narberhaus F. A mRNA-based thermosensor controls expression of rhizobial heat shock genes. Nucleic Acids Res. 2001;29(23):4800-7.

22. Chowdhury S, Maris C, Allain FH, Narberhaus F. Molecular basis for temperature sensing by an RNA thermometer. EMBO I. 2006:25(11):2487-97.

23. Breaker RR. Riboswitches and the RNA world. Cold Spring Harb Perspect Biol. 2012;4(2):003566. 
24. Henkin TM. Riboswitch rnas: using RNA to sense cellular metabolism. Genes Dev. 2008;22(24):3383-90.

25. Lin J-C, Thirumalai D. Gene regulation by riboswitches with and without negative feedback loop. Biophys J. 2012;103(11):2320-30.

26. Mironov AS, Gusarov I, Rafikov R, Lopez LE, Shatalin K, Kreneva RA, et al. Sensing small molecules by nascent RNA, a mechanism to control transcription in bacteria. Cell. 2002;111(5):747-56.

27. Nahvi A, Sudarsan N, Ebert MS, Zou X, Brown KL, Breaker RR. Genetic control by a metabolite binding mRNA. Chem Biol. 2002;9(9):1043.

28. Winkler W, Nahvi A, Breaker RR. Thiamine derivatives bind messenger RNAs directly to regulate bacterial gene expression. Nature. 2002;419(6910):952-6.

29. Thore S, Leibundgut M, Ban N. Structure of the eukaryotic thiamine pyrophosphate riboswitch with its regulatory ligand. Science. 2006;312(5777):1208-11.

30. Serganov A, Polonskaia A, Phan AT, Breaker RR, Patel DJ. Structural basis for gene regulation by a thiamine pyrophosphate-sensing riboswitch. Nature. 2006;441(7097):1167-71.

31. Montange RK, Batey RT. Structure of the s-adenosylmethionine riboswitch regulatory mRNA element. Nature. 2006;441(7097):1172-5.

32. Zuker M, Stiegler P. Optimal computer folding of large RNA sequences using thermodynamics and auxiliary information. Nucleic Acids Res. 1981;9:133-48.

33. McCaskill JS. The equilibrium partition function and base pair binding probabilities for RNA secondary structure. Biopolymers. 1990;29(6-7):1105-19.

34. Canny MD, Jucker FM, Kellogg E, Khvorova A, Jayasena SD, Pardi A. Fast cleavage kinetics of a natural hammerhead ribozyme. J Am Chem Soc. 2004;126(35):10848-9.

35. Ding $Y$, Chan CY, Lawrence CE. RNA secondary structure prediction by centroids in a Boltzmann weighted ensemble. RNA. 2005;11(8):1157-66.

36. Loh E, Dussurget O, Gripenland J, Vaitkevicius K, Tiensuu T, Mandin P, et al. A trans-acting riboswitch controls expression of the virulence regulator PrfA in Listeria monocytogenes. Cell. 2009;139(4):770-9.

37. Mellin JR, Tiensuu T, Becavin C, Gouin E, Johansson J, Cossart P. A riboswitch-regulated antisense RNA in listeria monocytogenes. Proc Natl Acad Sci U S A. 2013;110(32):13132-7.

38. Serganov A, Nudler E. A decade of riboswitches. Cell. 2013;152(1-2):17-24.

39. Serganov A, Yuan YR, Pikovskaya O, Polonskaia A, Malinina L, Phan AT, et al. Structural basis for discriminative regulation of gene expression by adenine- and guanine-sensing RANs. Chem Biol. 2004;11(12):1729-41.

40. Weinberg Z, Barrick JE, Yao Z, Roth A, Kim JN, Gore J, et al. Identification of 22 candidate structured RNAs in bacteria using the CMfinder comparative genomics pipeline. Nucleic Acids Res. 2007;35(14):4809-19.

41. Weinberg Z, Wang JX, Bogue J, Yang J, Corbino K, Moy RH, et al. Comparative genomics reveals 104 candidate structured RNAs from bacteria, archaea, and their metagenomes. Genome Biol. 2010;11(3):31.

42. Weinberg Z, Regulski EE, Hammond MC, Barrick JE, Yao Z, Ruzzo WL, et al. The aptamer core of SAM-IV riboswitches mimics the ligand-binding site of SAM-I riboswitches. RNA. 2008;14(5):822-8.

43. Edwards T. E, Ferre-D'Amare A. R. Crystal structures of the thi-box riboswitch bound to thiamine pyrophosphate analogs reveal adaptive RNA-small molecule recognition. Structure. 2006;14(9):1459-68.

44. Bryngelson JD, Onuchic JN, Socci ND, Wolynes PG. Funnels, pathways, and the energy landscape of protein folding: a synthesis. Proteins. 1995;21(3):167-95.

45. Quarta G, Kim N, Izzo JA, Schlick T. Analysis of riboswitch structure and function by an energy landscape framework. J Mol Biol. 2009;393:993-1003.

46. Quarta G, Sin K, Schlick T. Dynamic energy landscapes of riboswitches help interpret conformational rearrangements and function. PLoS Comput Biol. 2012;8(2):1002368.

47. Ritz J, Martin JS, Laederach A. Evolutionary evidence for alternative structure in RNA sequence co-variation. PLoS Comput Biol. 2013;9(7):1003152.

48. Giegerich R, Haase D, Rehmsmeier M. Prediction and visualization of structural switches in RNA. Pac Symp Biocomput. 1999126-37. Using Smart Source Parsing.

49. Steffen P, Voss B, Rehmsmeier M, Reeder J, Giegerich R. RNAshapes: an integrated RNA analysis package based on abstract shapes. Bioinformatics. 2006;22(4):500-3. Using Smart Source Parsing Feb 15; Epub 2005 Dec 15.

50. Freyhult E, Moulton V, Clote P. RNAbor: a web server for RNA structural neighbors. Nucleic Acids Res. 2007;35(Web Server issue):305-9.

51. Freyhult E, Moulton V, Clote P. Boltzmann probability of RNA structural neighbors and riboswitch detection. Bioinformatics. 2007;23(16):2054-62. Using Smart Source Parsing Aug 15; Epub 2007 Jun 14.

52. Senter E, Sheikh S, Dotu I, Ponty Y, Clote P. Using the fast fourier transform to accelerate the computational search for RNA conformational switches. PLoS One. 2012;7(12):19. Using Smart Source Parsing e50506. doi:10.1371/journal.pone.0050506. Epub 2012 Dec.

53. Shannon C. E. A mathematical theory of communication. Bell Syst Technical J. 1948;27:379-423.

54. Huynen M, Gutell R, Konings D. Assessing the reliability of RNA folding using statistical mechanics. J Mol Biol. 1997;267:1104-12.

55. Manzourolajdad A, Wang Y, Shaw TI, Malmberg RL. Information-theoretic uncertainty of SCFG-modeled folding space of the non-coding RNA. J Theor Biol. 2013;318(0):140-63.

56. Sukosd Z, Knudsen B, Anderson JW, Novak A, Kjems J, Pedersen CN. Characterising RNA secondary structure space using information entropy. BMC Bioinformatics. 2013;14(2):1471-2105. Using Smart Source Parsing Suppl S22. doi:10.1186/14-S2-S22. Epub 2013 Jan 21.

57. Sukosd Z, Knudsen B, Vaerum M, Kjems J, Andersen ES. Multithreaded comparative RNA secondary structure prediction using stochastic context-free grammars. BMC Bioinformatics. 2011;12(103):1471-2105. Using Smart Source Parsing Apr 18; doi:10.1186/12-103.

58. Hofacker IL. Vienna RNA secondary structure server. Nucleic Acids Res. 2003;31(13):3429-31.

59. Rivas $E$, Lang R, Eddy SR. A range of complex probabilistic models for RNA secondary structure prediction that includes the nearest-neighbor model and more. RNA. 2012;18(2):193-212. Using Smart Source Parsing Feb; doi:10.1261/rna.030049.111. Epub 2011 Dec 22. 
60. Altschul SF, Erickson BW. Significance of nucleotide sequence alignments: a method for random sequence permutation that preserves dinucleotide and codon usage. Mol Biol Evol. 1985;2:526-38.

61. Wang Y, Manzour A, Shareghi P, Shaw TI, Li YW, Malmberg RL, et al. Stable stem enabled shannon entropies distinguish non-coding rnas from random backgrounds. BMC Bioinformatics. 2012;13(Suppl 5):1.

62. Sato K, Hamada M, Asai K, Mituyama T. cENTROIDFOLD, a web server for RNA secondary structure prediction. Nucleic Acids Res. 2009:37(Web Server issue):277-80.

63. Tran TT, Zhou F, Marshburn S, Stead M, Kushner SR, Xu Y. De novo computational prediction of non-coding RNA genes in prokaryotic genomes. Bioinformatics. 2009;25(22):2897-905.

64. Naclerio G, Baccigalupi L, Zilhao R, De Felice M, Ricca E. Erratum. Bacillus subtilis spore coat assembly requires cotH gene expression. J Bacteriol. 1996;178(21):6407.

65. Giglio R, Fani R, Isticato R, De Felice M, Ricca E, Baccigalupi L. Organization and evolution of the $\cot G$ and $\cot H$ genes of Bacillus subtilis. J Bacteriol. 2011;193(23):6664-73.

66. Mrazek J, Xie S. Pattern locator: a new tool for finding local sequence patterns in genomic DNA sequences. Bioinformatics. 2006;22(24):3099-100.

67. Cloutier SC, Wang S, Ma WK, Petell CJ, Tran EJ. Long noncoding RNAs promote transcriptional poising of inducible genes. PLoS Biol. 2013;11(11):1001715.

68. Chan PP, Lowe TM. GtRNAdb: a database of transfer RNA genes detected in genomic sequence. Nucleic Acids Res 2009;37(Database issue):93-7.

69. Kolter R, Yanofsky C. Attenuation in amino acid biosynthetic operons. Annu Rev Genet. 1982;16:113-34.

70. Lukashin AV, Borodovsky M. Genemark.hmm: new solutions for gene finding. Nucleic Acids Res. 1998;26(4):1 107-15.

71. Salzberg SL, Delcher AL, Kasif S, White O. Microbial gene identification using interpolated Markov models. Nucleic Acids Res. 1998;26(2):544-8.

72. Delcher AL, Harmon D, Kasif S, White O, Salzberg SL. Improved microbial gene identification with glimmer. Nucleic Acids Res. 1999;27(23):4636-41.

73. Lee EJ, Groisman EA. Control of a Salmonella virulence locus by an ATP-sensing leader messenger RNA. Nature. 2012;486(7402):271-5.

74. Ferre-D'Amare AR. The glmS ribozyme: use of a small molecule coenzyme by a gene-regulatory RNA. Q Rev Biophys. 2010:43(4):423-47.

75. Winkler WC, Nahvi A, Roth A, Collins JA, Breaker RR. Control of gene expression by a natural metabolite-responsive ribozyme. Nature. 2004;428(6980):281-6.

76. Barrick JE, Corbino KA, Winkler WC, Nahvi A, Mandal M, Collins J, et al. New RNA motifs suggest an expanded scope for riboswitches in bacterial genetic control. Proc Natl Acad Sci U S A. 2004;101(17):6421-6.

77. Knudsen B, Hein J. RNA secondary structure prediction using stochastic context-free grammars and evolutionary history. Bioinformatics. 1999;15:446-54.

78. Knudsen B, Hein J. Pfold: RNA secondary structure prediction using stochastic context-free grammars. Nucl Acids Res. 2003:31:3423-8.

79. Dowell RD, Eddy SR. Evaluation of several lightweight stochastic context-free grammars for RNA secondary structure prediction. BMC Bioinformatics. 2004;5:71.

80. Singh P, Bandyopadhyay P, Bhattacharya S, Krishnamachari A, Sengupta S. Riboswitch detection using profile hidden markov models. BMC Bioinformatics. 2009;10:325.

81. Sayers EW, Barrett T, Benson DA, Bryant SH, Canese K, Chetvernin V, et al. Database resources of the national center for biotechnology information. Nucleic Acids Res. 2009;37(Database issue):21.

82. Benson DA, Karsch-Mizrachi I, Lipman DJ, Ostell J, Sayers EW. Genbank. Nucleic Acids Res. 2009;37(Database issue):21.

83. Taboada B, Ciria R, Martinez-Guerrero CE, Merino E. ProOPDB, Prokaryotic operon database. Nucleic Acids Res. 2012;40(Database issue):627-31.

84. Salgado H, Peralta-Gil M, Gama-Castro S, Santos-Zavaleta A, Muniz-Rascado L, Garcia-Sotelo JS, et al. RegulonDB v8.0: omics data sets, evolutionary conservation, regulatory phrases, cross-validated gold standards and more. Nucleic Acids Res. 2013;41(Database issue):203-13.

85. Price MN, Alm EJ, Arkin AP. Interruptions in gene expression drive highly expressed operons to the leading strand of DNA replication. Nucleic Acids Res. 2005;33(10):3224-34.

86. Price MN, Huang KH, Alm EJ, Arkin AP. A novel method for accurate operon predictions in all sequenced prokaryotes. Nucleic Acids Res. 2005;33(3):880-92.

87. Rivas E, Eddy SR. A dynamic programming algorithm for RNA structure prediction including pseudoknots. J Mol Biol. 1999;285(5):2053-68.

88. Reeder J, Steffen P, Giegerich R. pknotsrg: RNA pseudoknot folding including near-optimal structures and sliding windows. Nucleic Acids Res. 2007;35(Web Server issue):320-4.

89. Griffiths-Jones S, Moxon S, Marshall M, Khanna A, Eddy SR, Bateman A. Rfam: annotating non-coding RNAs in complete genomes. Nucleic Acids Res. 2005;33:121-4.

90. Gluick TC, Gerstner RB, Draper DE. Effects of $\mathrm{mg} 2+, \mathrm{k}+$, and $\mathrm{h}+$ on an equilibrium between alternative conformations of an RNA pseudoknot. J Mol Biol. 1997;270(3):451-63.

91. Schlax PJ, Xavier KA, Gluick TC, Draper DE. Translational repression of the Escherichia coli al pha operon mRNA importance of an mRNA conformational switch and a ternary entrapment complex. J Biol Chem. 2001;276(42): 38494-501.

92. Watson PY, Fedor MJ. The $y d a O$ motif is an ATP-sensing riboswitch in Bacillus subtilis. Nat Chem Biol. 2012;8(12): 963-5.

93. Smith KD, Lipchock SV, Ames TD, Wang J, Breaker RR, Strobel SA. Structural basis of ligand binding by a c-di-gmp riboswitch. Nat Struct Mol Biol. 2009;16(12):1218-23.

94. Vitreschak AG, Rodionov DA, Mironov AA, Gelfand MS. Regulation of the vitamin b12 metabolism and transport in bacteria by a conserved RNA structural element. RNA. 2003;9(9):1084-97.

95. Ravnum S, Andersson DI. An adenosyl-cobalamin (coenzyme- b12)-repressed translational enhancer in the cob MRNA of Salmonella typhimurium. Mol Microbiol. 2001;39(6):1585-94. 
96. Ames TD, Breaker RR. Bacterial aptamers that selectively bind glutamine. RNA Biol. 2011;8(1):82-9.

97. Baker JL, Sudarsan N, Weinberg Z, Roth A, Stockbridge RB, Breaker RR. Widespread genetic switches and toxicity resistance proteins for fluoride. Science. 2012;335(6065):233-5.

98. Ren A, Rajashankar KR, Patel DJ. Fluoride ion encapsulation by Mg2+ ions and phosphates in a fluoride riboswitch Nature. 2012;486(7401):85-9.

99. Serganov A, Huang L, Patel DJ. Coenzyme recognition and gene regulation by a flavin mononucleotide riboswitch. Nature. 2009:458(7235):233-7.

100. Vicens Q, Mondragon E, Batey RT. Molecular sensing by the aptamer domain of the fmn riboswitch: a general model for ligand binding by conformational selection. Nucleic Acids Res. 2011;39(19):8586-98.

101. Winkler WC, Cohen-Chalamish S, Breaker RR. An mRNA structure that controls gene expression by binding FMN Proc Natl Acad Sci USA. 2002;99(25):15908-13.

102. Cochrane JC, Lipchock SV, Strobel SA. Structural investigation of the G/mS ribozyme bound to its catalytic cofactor. Chem Biol. 2007;14(1):97-105.

103. Klein DJ, Ferre-D'Amare AR. Crystallization of the glmS ribozyme-riboswitch. Methods Mol Biol. 2009;540:129-39.

104. Mandal M, Lee M, Barrick JE, Weinberg Z, Emilsson GM, Ruzzo WL, et al. A glycine-dependent riboswitch that uses cooperative binding to control gene expression. Science. 2004;306(5694):275-9.

105. Kwon M, Strobel SA. Chemical basis of glycine riboswitch cooperativity. RNA. 2008;14(1):25-34.

106. Butler EB, Xiong Y, Wang J, Strobel SA. Structural basis of cooperative ligand binding by the glycine riboswitch. Chem Biol. 2011;18(3):293-8.

107. Martick M, Scott WG. Tertiary contacts distant from the active site prime a ribozyme for catalysis. Cell. 2011;126(2): $309-20$.

108. Perreault J, Weinberg Z, Roth A, Popescu O, Chartrand P, Ferbeyre G, et al. Identification of hammerhead ribozymes in all domains of life reveals novel structural variations. PLoS Comput Biol. 2011;7(5):1002031.

109. Serganov A, Huang L, Patel DJ. Structural insights into amino acid binding and gene control by a lysine riboswitch. Nature. 2008:455(7217):1263-7.

110. Garst AD, Heroux A, Rambo RP, Batey RT. Crystal structure of the lysine riboswitch regulatory mRNA element. J Biol Chem. 2008;283(33):22347-51.

111. Regulski EE, Moy RH, Weinberg Z, Barrick JE, Yao Z, Ruzzo WL, et al. A widespread riboswitch candidate that controls bacterial genes involved in molybdenum cofactor and tungsten cofactor metabolism. Mol Microbiol. 2008;68(4):918-32.

112. Meyer MM, Roth A, Chervin SM, Garcia GA, Breaker RR. Confirmation of a second natural preQ1 aptamer class in Streptococcaceae bacteria. RNA. 2008;14(4):685-95.

113. Klein DJ, Edwards TE, Ferre-D'Amare AR. Cocrystal structure of a class I preQ1 riboswitch reveals a pseudoknot recognizing an essential hypermodified nucleobase. Nat Struct Mol Biol. 2009;16(3):343-4.

114. Batey RT, Gilbert SD, Montange RK. Structure of a natural guanine-responsive riboswitch complexed with the metabolite hypoxanthine. Nature. 2004;432(7015):411-5.

115. Edwards AL, Reyes FE, Heroux A, Batey RT. Structural basis for recognition of s-adenosylhomocysteine by riboswitches. RNA. 2010;16(11):2144-55.

116. Grundy FJ, Henkin TM. The S box regulon: a new global transcription termination control system for methionine and cysteine biosynthesis genes in gram-positive bacteria. Mol Microbiol. 1998;30(4):737-49.

117. Tomsic J, McDaniel BA, Grundy FJ, Henkin TM. Natural variability in S-adenosylmethionine (SAM,-dependent riboswitches: S-box elements in Bacillus subtilis exhibit differential sensitivity to SAM in vivo and in vitro. J Bacteriol. 2008;190(3):823-33.

118. Lu C, Ding F, Chowdhury A, Pradhan V, Tomsic J, Holmes WM, et al. SAM recognition and conformational switching mechanism in the Bacillus subtilis yitJ S box/SAM-I riboswitch. J Mol Biol. 2010;404(5):803-18.

119. Boyapati VK, Huang W, Spedale J, Aboul-Ela F. Basis for ligand discrimination between on and off state riboswitch conformations: the case of the SAM-I riboswitch. RNA. 2012;18(6):1230-43.

120. Corbino KA, Barrick JE, Lim J, WelzR, Tucker BJ, Puskarz I, et al. Evidence for a second class of s-adenosylmethionine riboswitches and other regulatory RNA motifs in alpha-proteobacteria. Genome Biol. 2005;6(8):70.

121. Fuchs RT, Grundy FJ, Henkin TM. The S(MK, box is a new SAM-binding RNA for translational regulation of sam synthetase. Nat Struct Mol Biol. 2006;13(3):226-33.

122. Lu C, Smith AM, Fuchs RT, Ding F, Rajashankar K, Henkin TM, et al. Crystal structures of the SAM-III/S(MK, riboswitch reveal the SAM-dependent translation inhibition mechanism. Nat Struct Mol Biol. 2008;15(10):1076-83.

123. Wilson RC, Smith AM, Fuchs RT, Kleckner IR, Henkin TM, Foster MP. Tuning riboswitch regulation through conformational selection. J Mol Biol. 2011;405(4):926-38.

124. Poiata E, Meyer MM, Ames TD, Breaker RR. A variant riboswitch aptamer class for S-adenosylmethionine common in marine bacteria. RNA. 2009;15(11):2046-56.

125. Meyer MM, Ames TD, Smith DP, Weinberg Z, Schwalbach MS, Giovannoni SJ, et al. Identification of candidate structured RNAs in the marine organism 'Candidatus Pelagibacter ubique'. BMC Genomics. 2009;10:268.

126. Ames TD, Rodionov DA, Weinberg Z, Breaker RR. A eubacterial riboswitch class that senses the coenzyme tetrahydrofolate. Chem Biol. 2010;17(7):681-5.

127. Huang L, Ishibe-Murakami S, Patel DJ, Serganov A. Long-range pseudoknot interactions dictate the regulatory response in the tetrahydrofolate riboswitch. Proc Natl Acad Sci USA. 2011;108(36):14801-6.

128. Winkler W, Nahvi A, Breaker RR. Thiamine derivatives bind messenger RNAs directly to regulate bacterial gene expression. Nature. 2002;419(6910):952-6.

129. Nudler E. Flipping riboswitches. Cell. 2006;126(1):19-22.

130. Haller A, Altman RB, Souliere MF, Blanchard SC, Micura R. Folding and ligand recognition of the TPP riboswitch aptamer at single-molecule resolution. Proc Natl Acad Sci USA. 2013;110(11):4188-93.

131. Neidhardt FC, Vol. 1. Escherichia Coli and Salmonella, Cellular and Molecular Biology, 2nd edn. Washington, DC: ASM Press; 1996, pp. 1263-1286. 
132. Babitzke P, Gollnick P. Posttranscription initiation control of tryptophan metabolism in Bacillus subtilis by the trp RNA-binding attenuation protein (TRAP): anti-TRAP, and RNA structure. J Bacteriol. 2001;183(20):5795-802.

133. Babitzke P, Schaak J, Yakhnin AV, Bevilacqua PC. Role of RNA structure in transcription attenuation in Bacillus subtilis, the trpEDCFBA operon as a model system. Methods Enzymol. 2003;371:392-404.

134. Winkler WC, Nahvi A, Sudarsan N, Barrick JE, Breaker RR. An mRNA structure that controls gene expression by binding s-adenosylmethionine. Nat Struct Biol. 2003;10(9):701-7.

Submit your next manuscript to BioMed Central and take full advantage of:

- Convenient online submission

- Thorough peer review

- No space constraints or color figure charges

- Immediate publication on acceptance

- Inclusion in PubMed, CAS, Scopus and Google Scholar

- Research which is freely available for redistribution 